Portland State University

PDXScholar

$12-4-1992$

\title{
Semiconductor P-N Junction Space Charge Region Capacitance
}

Mohammad Humayun Habib

Portland State University

Follow this and additional works at: https://pdxscholar.library.pdx.edu/open_access_etds

Part of the Electrical and Electronics Commons

Let us know how access to this document benefits you.

\section{Recommended Citation}

Habib, Mohammad Humayun, "Semiconductor P-N Junction Space Charge Region Capacitance" (1992). Dissertations and Theses. Paper 4589.

https://doi.org/10.15760/etd.6473

This Thesis is brought to you for free and open access. It has been accepted for inclusion in Dissertations and Theses by an authorized administrator of PDXScholar. Please contact us if we can make this document more accessible: pdxscholar@pdx.edu. 
AN ABSTRACT OF THE THESIS OF Mohammad Humayun Habib for the Master of Science in Electrical Engineering presented on December 04, 1992.

Title: $\quad$ Semiconductor P-N Junction Space Charge Region Capacitance.

\section{APPROVED BY THE MEMBERS OF THE THESIS COMMITTEE:}
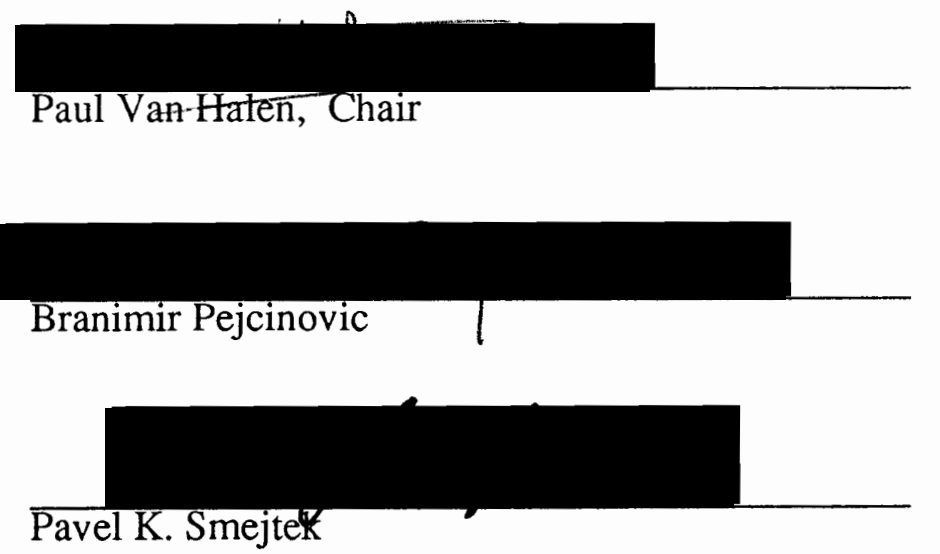

The classical capacitance voltage characteristics based on the depletion approximation, is adequate at reverse bias, but introduces errors at high forward bias. Because of its inherent simplicity and compactness this classical depletion model is well studied and widely used in circuit simulators. In this work, a new model for the semiconductor space charge region (SCR) capacitance, based on physical justification, will be derived. This new model takes three input parameters, $C_{o}, V_{b i}$ and $m$, thus eliminating the fitting parameter FC currently used in SPICE. This new model is applicable for any applied voltage and will be compared with the SCR capacitance extracted from the numerical device simulator PISCES, and with the SCR capacitance models proposed by Gummel and Poon and by DeGraaff and Klaassen. 
SEMICONDUCTOR P-N JUNCTION SPACE CHARGE

REGION CAPACITANCE

\author{
by \\ MOHAMMAD HUMAYUN HABIB
}

A thesis submitted in partial fulfillment of the requirements for the degree of

\author{
MASTER OF SCIENCE \\ in \\ ELECTRICAL ENGINEERING
}

Portland State University

1993 
TO THE OFFICE OF THE GRADUATE STUDIES:

The members of the committee approve the thesis of Mohammad Humayun Habib presented on December 04, 1992.
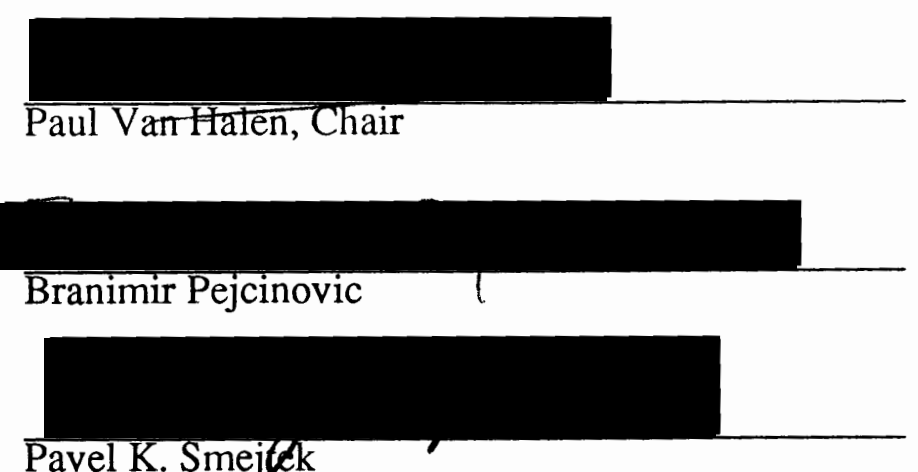

\section{APPROVED:}

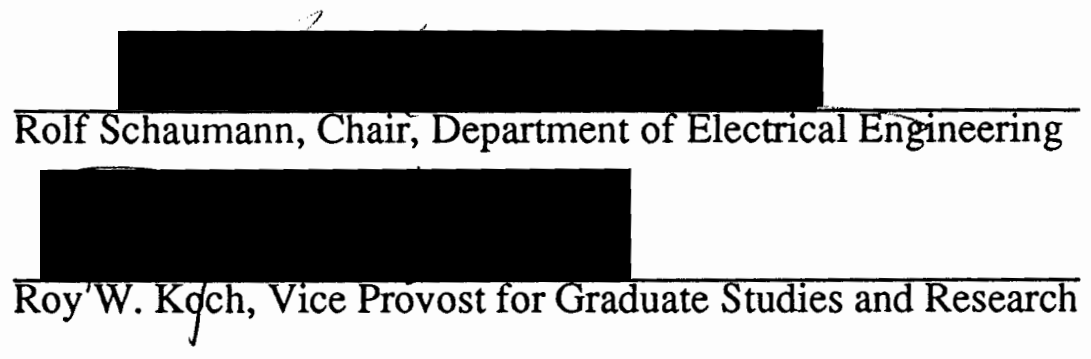




\section{TABLE OF CONTENTS}

\section{CHAPTER}

II.1 Potential and Carrier Distribution of a p-n Junction . . . . . . . . . . . . . . 4

II.2 Definition of Capacitance of a $\mathrm{p}-\mathrm{n}$ Junction .

IV.1 Net Charge Method ................ 20

IV.2 Net Carrier Method ................ 27

IV.3 AC Small-Signal Analysis Method .......... 31

IV.4 Capacitance vs Quasi-Fermi

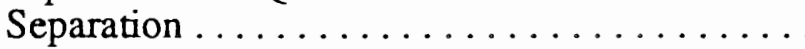

IV.5 Problems in Extraction of

Capacitance by PISCES . . . . . . . . . . . . $\quad 39$

NEW CAPACITANCE MODEL $\ldots \ldots \ldots \ldots \ldots \ldots$

V.1 Derivation of a New Model for SCR Capacitance .................

V.2 Comparison of New Model with Previous Model ........................ 


\section{LIST OF FIGURES}

FIGURE

PAGE

1. Schematic and energy bad diagram of a p-n junction. ........ 5

2. Percentage of error associated with Eq. (3.5) for asymmetrical

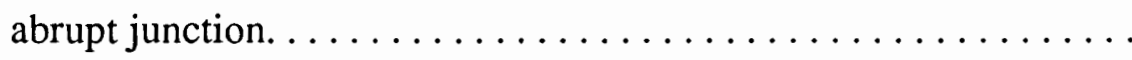

3. Schematic of $p-n$ junction device used for the device simulator PISCES . . . . . . . . . . . . . . . . . . .

4. Distribution of electron and hole concentration for the device in Figure 3, extracted by PISCES for a symmetrical abrupt doping $N_{A}=N_{D}=10^{17} \mathrm{~cm}^{-3}$ and for applied voltage $V_{\text {app }}=0 \ldots$

5. Space charge distribution $\left(\mathrm{cm}^{-3}\right), \rho(x)$, extracted by PISCES for the $\mathrm{p}-\mathrm{n}$ junction device shown in Figure $3 \ldots \ldots \ldots \ldots \ldots$

6. Integrated result of the space charge in Figure 5 from contact to contact for the junction shown in Figure $3 \ldots \ldots \ldots \ldots \ldots$

7. Total charge $\mathrm{Q}$ in the space charge region versus applied voltage for the device shown in Figure 3, extracted by PISCES.......

8. Derivative taken by Tekspice of the curve shown in Figure 7 with respect to the applied voltage, which is the space charge region

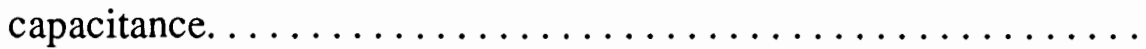

9. Space Charge distribution, extracted from PISCES for the $p-n$ junction device shown in Figure $3 . \ldots \ldots \ldots \ldots \ldots \ldots . . . .$.

10. Distribution of net carrier concentration extracted by PISCES for $V_{a p p}=0$, for the device shown in Figure 3 
11. Integrated net carrier concentration extracted by PISCES for $V_{a p p}=0$, for the device shown in Figure $3 . \ldots \ldots \ldots \ldots$

12. Integrated net carrier concentration extracted by PISCES, for the device shown in Figure $3 . \ldots \ldots \ldots \ldots \ldots \ldots \ldots$

13. Derivative of the integrated net carrier concentration taken by Tekspice for the curve in Figure 12, which is the capaci-

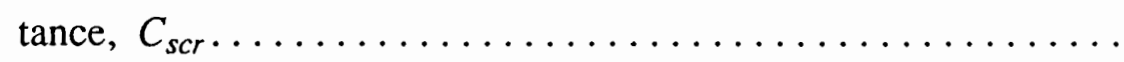

14. Capacitance extracted by PISCES from ac small-signal analysis. .

15. Comparison of capacitances extracted from PISCES by ac smallsignal analysis and by net charge method. . . . . . . . . . .

16. Distribution of hole and electron quasi-Fermi level for $V_{a p p}=0.3$ volts, extracted from PISCES. . . . . . . . . . . . .

17. Distribution of hole and electron quasi-Fermi level and potential for $V_{a p p}=1.0$ volts. $\ldots \ldots \ldots \ldots \ldots \ldots \ldots \ldots \ldots \ldots \ldots \ldots \ldots \ldots$

18. Quasi-Fermi level separation at the junction $\phi_{S C R}$ versus $V_{a p p}$, extracted by PISCES, for the p-n junction device shown

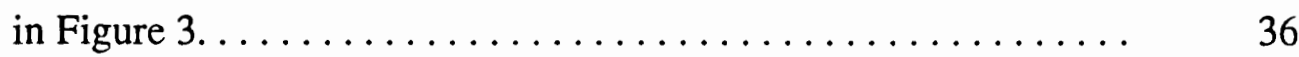

19. Total space charge $Q$ versus quasi-Fermi separation. . . . . . . 37

20. Derivative of the total space charge with respect to quasiFermi level separation, taken by Tekspice for the curve in

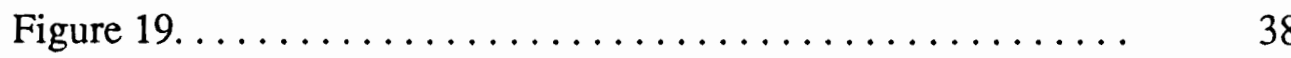

21. Enlarged peak of Figure 20 to compare the location and value of the peak with that found by Van Den Biesen [15]. . . . . . . .

22. Distribution of $p(x)$ and $n(x)$ for an asymmetrical abrupt junction, $N_{A}=10^{18}$ and $N_{D}=10^{16} \mathrm{~cm}^{-3}$ and a junction depth of 0.5 micron, for $V_{a p p}=0 \ldots \ldots \ldots \ldots \ldots \ldots$ 
23. Space charge distribution $\left(\mathrm{cm}^{-3}\right), \rho(x)$, extracted by PISCES

for asymmetrical abrupt junction shown in Figure 22, for $V_{a p p}=0$. .

24. Distribution of net carrier concentration in absolute value, extracted by PISCES for $V_{a p p}=0$, for asymmetrical abrupt junction shown in Figure $22 \ldots \ldots \ldots \ldots \ldots \ldots \ldots \ldots$

25. Comparison between the present SPICE model Eq. (1.1) and (1.2) for SCR capacitance and actual capacitance extracted by PISCES ..

26. Comparison between the model proposed by Van Halen, Eq. (5.1) and the actual capacitance extracted by PISCES. . . . . . . . .

27. Comparison of the new model Eq. (5.3) with the actual capacitance extracted by PISCES for $N_{A}=N_{D}=10^{15} \mathrm{~cm}^{-3} \ldots \ldots \ldots$.

28. Comparison of the new model Eq. (5.3) with the actual capacitance extracted by PISCES for $N_{A}=N_{D}=10^{19} \mathrm{~cm}^{-3} \ldots \ldots \ldots$.

29. Comparison of the new model Eq. (5.3) with the actual capacitance extracted by PISCES for linear graded profile with $a=10^{18} \mathrm{~cm}^{-4} \ldots$

30. Comparison of the new model Eq. (5.3) with the actual capacitance extracted by PISCES for linear graded profile with $a=10^{24} \mathrm{~cm}^{-4} \ldots$

31. Comparison of the new model Eq. (5.3) with the actual capacitance extracted by PISCES for $N_{A}=10^{17}$ and $N_{D}=10^{15} \mathrm{~cm}^{-3} \ldots$.

32. Comparison of the new model Eq. (5.3) with the actual capacitance extracted by PISCES for $N_{A}=10^{18}$ and $N_{D}=10^{14} \mathrm{~cm}^{-3} \ldots$. 51

33. Comparison of the new model Eq. (5.3) with the actual capacitance extracted by PISCES for $N_{A}=10^{19}$ and $N_{D}=5 \times 10^{16} \mathrm{~cm}^{-3} . \quad 52$ 34. Comparison of the new model with VH, GP and GK model. ...... 54

35. Comparison of the GP and GK model and the new model with PISCES result for linear graded profile with $a=10^{22} \mathrm{~cm}^{-4} \ldots \ldots$. 
36. Comparison of the VH model and the new model with PISCES result for linear graded profile with $a=10^{22} \mathrm{~cm}^{-4} \ldots \ldots \ldots$

37. Enlarged peak of Figure $34 \ldots \ldots \ldots \ldots \ldots \ldots \ldots \ldots \ldots \ldots$ 


\section{CHAPTER I}

\section{INTRODUCTION}

Very large scale integration (VLSI) fabrication techniques for semiconductor devices have increased the demand for more accurate device models that are used in computer simulation. The capacitance of $\mathrm{p}-\mathrm{n}$ junction plays a significant role in switching speed and frequency response, particularly for silicon VLSI bipolar devices. The recent advances in bipolar technology have increased the role of forward voltage capacitance of the emitter-base junction; the increased doping concentration in the base region reduces the relative importance of the quasi neutral region capacitance or diffusion capacitance. Further, because the quasi neutral region component of the total capacitance is proportional to the square of the intrinsic carrier concentration $n_{i}$, or $\exp \left(-E_{g} / \mathrm{kT}\right)$, where $E_{g}$ is the energy band gap and $\mathrm{kT}$ is the thermal energy, the junction capacitance tends to dominate for semiconductors having large energy band gap ( such as in Gallium Arsenide based devices), or operating at low temperatures.

The well known semiconductor space charge region capacitance equation

$$
C=\left[\frac{C_{o}}{\left.1-\frac{V_{a p p}}{V_{b i}}\right]^{m}}\right.
$$

where $C_{o}$ is the space charge region capacitance at zero applied voltage, $V_{b i}$ is the built-in potential, $V_{a p p}$ is the applied voltage and $m$ is the grading coefficient which varies between 0.33 and 0.5 , depending on whether the junction is linearly graded or abrupt. This model is incorporated in circuit simulators such as SPICE. 
The major problem of this expression is the singularity, which occurs at $V_{a p p}=V_{b i}$. Numerical circuit simulators like SPICE, prevent this problem from occurring by introducing a new parameter (FC in SPICE) and a linear approximation to the previous capacitance curve such that for. $V_{a p p}>F C . V_{b i}$

$$
C=C_{o}\left[(1-F C)^{-m}+\frac{m(1-F C)^{-m}}{V_{b i}(1-F C)}\left(V_{a p p}-F C \cdot V_{b i}\right)\right]
$$

The combination of Eq. (1.1) and (1.2) avoids the singularity in the capacitance-voltage characteristics, at the expense of a new fitting parameter FC and a nonphysical approximation of the junction capacitance, at high forward bias. The junction capacitance in fact peaks at a voltage lower than the built-in potential and then decreases exponentially towards zero at higher voltages.

There have been many attempts over the past three decades to characterize the $p-n$ junction region capacitance. Though instructive and useful, these attempts have provided only a partial description. The nonlinearity associated with the junction region has made this problem more difficult, particularly for high forward bias.

In this work the behavior of the space charge region capacitance at high forward bias is treated in detail. A new space charge region capacitance model, based on physical justification, and applicable for any applied voltage, has been derived. This new model needs only three input parameters $C_{o}, V_{b i}$ and $m$, thus eliminating the parameter FC in SPICE. These three parameters can be measured for any device and also can be calculated if the doping profile and the junction geometry is known.

Organization of this paper is as follows: In Chapter II, the basic properties of the $p-n$ junction space charge region and various components of the capacitances associated with the p-n junction device are described. In Chapter III, the previous work on space charge region capacitance is discussed. In Chapter IV, the present approach, how the 
space charge region capacitance is obtained from the numerical device simulator PISCES, is described in detail. As there is no direct method to extract capacitance from PISCES, the problem associated in determining the space charge region capacitance is also described. In Chapter $\mathrm{V}$, the new model is derived for three cases, symmetrical abrupt, asymmetrical abrupt and linear graded junction. This model is then compared with the capacitances obtained from PISCES and the other model proposed in the literature. Chapter VI concludes with a discussion of the results. 


\section{CHAPTER II}

\section{PROPERTIES OF A P-N JUNCTION}

\section{II.1 POTENTIAL AND CARRIER DISTRIBUTION OF A P-N JUNCTION}

Most semiconductor devices can be studied by assuming that space charge neutrality exists far from any discontinuity or rapid change of impurity concentration versus distance, and then applying Poisson's equation only in the region of such discontinuities. So, analysis of the $\mathrm{p}-\mathrm{n}$ junction space charge region always involves Poisson's equation, which in the absence of charge on traps in the forbidden gap is,

$$
\frac{d^{2} \psi}{d x^{2}}=\frac{q}{\varepsilon}\left[N_{D}-N_{A}+p(x)-n(x)\right]
$$

for a one dimensional model, where $\psi$ is the electrostatic potential across the junction, $q$ is the electron charge, $\varepsilon$ is the dielectric permittivity, $N_{D}$ is the donor impurity doping concentration, $N_{A}$ is the acceptor impurity doping concentration, $p(x)$ is the hole concentration and $n(x)$ is the electron concentration. In general nonlinear relations describe $p(x)$ and $n(x)$ in terms of the electrostatic potential $\psi$ and electrochemical potential (or quasi-Fermi potential) $\phi_{n}$ and $\phi_{p}$, as

and

$$
p(x)=n_{i} \exp \left[\frac{\psi-\phi_{p}}{V_{t}}\right]
$$

$$
n(x)=n_{i} \exp \left[\frac{\phi_{n}-\psi}{V_{t}}\right]
$$


where $n_{i}$ is the intrinsic carrier concentration and $V_{t}$ is the thermal voltage $V_{l}=\frac{k T}{q}$.

Equations (2.2) and (2.3) hold only for nondegenerate electron and hole concentrations, for which Boltzmann statistics adequately approximate Fermi-Dirac statistics. A qualitative one dimensional structure of a p-n junction diode and its energy band diagram is illustrated in Figure 1, where $E_{c}$ is the conduction band, $E_{v}$ is the valence band, $V$ is the voltage across the junction which is also the separation of the quasi-Fermi potential in the space charge region and the junction barrier height equals $V_{b i}-V$. Here quasiFermi potentials are assumed to be nearly position independent across the space charge region. This approximation introduces negligible error provided that each quasi-Fermi potential varies by less than $V_{t}$ across the space charge region, a condition that holds even for high current densities.

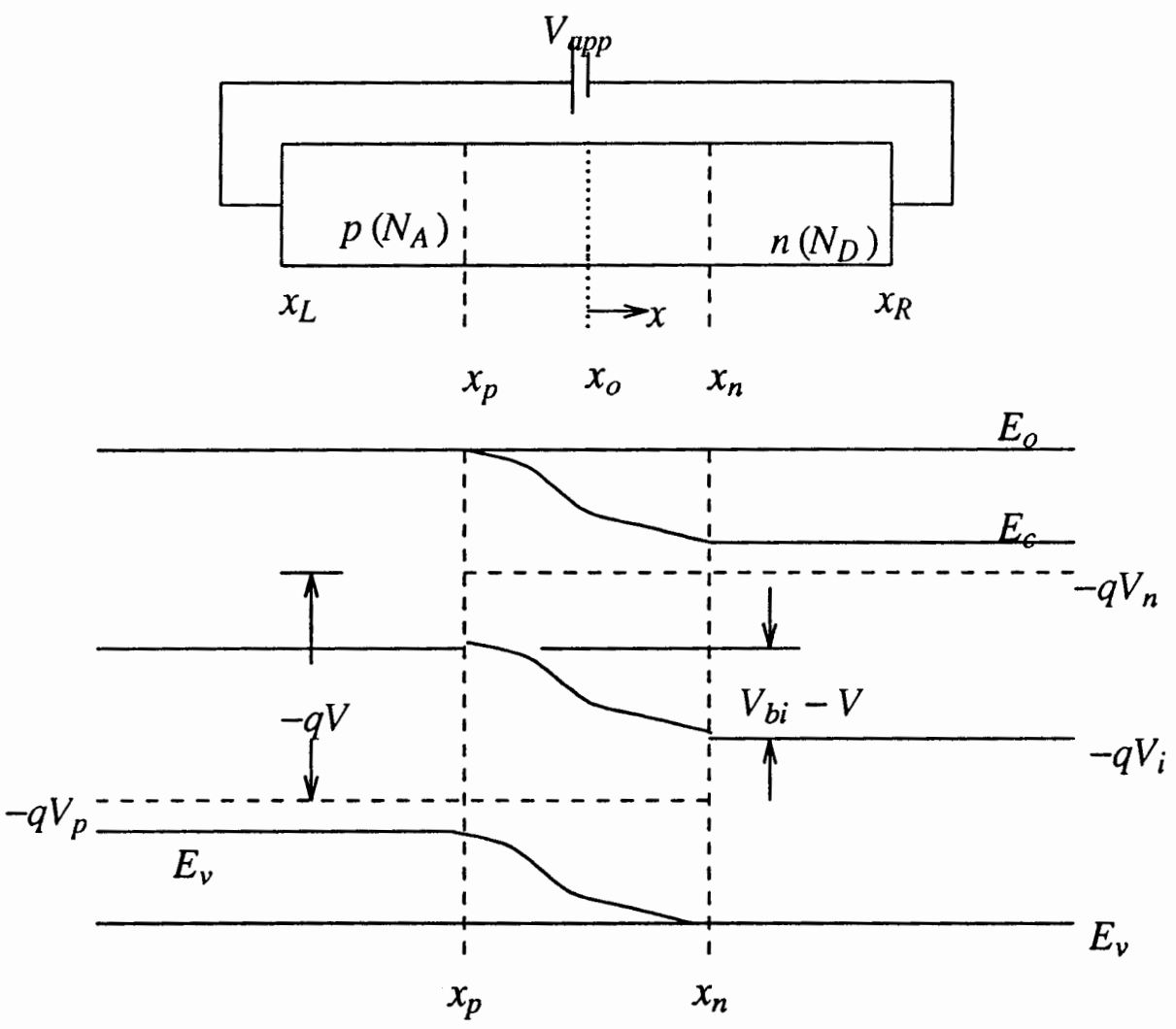

Figure 1. Schematic and energy bad diagram of a p-n junction. 
The analysis of the space charge region becomes straight forward when the junction is under reverse bias [2],[3]. For such bias, the commonly used depletionapproximation is adequate. The space charge region ( $\mathrm{SCR}$ ) is depleted of free electrons and holes because of the high electric field. Elimination of negligible electrons and holes linearizes Poisson's equation and the analysis of the SCR, which includes determining its thickness and its capacitance, can be carried out by integrating the linearized Poisson's equation.

\section{II.2 DEFINITION OF CAPACITANCE OF A P-N JUNCTION}

Here the definition of the junction capacitance and its subdivision into depletion, diffusion and neutral capacitance is explored. Moreover the spatial distribution of charges and associated capacitances is illustrated.

In the following a $\mathrm{p}$-n diode will be considered with contacts at $x=x_{L}$ on the $\mathrm{p}$ side and $x=x_{R}$ on the $\mathrm{n}$ side. The metallurgical junction is situated at $x=0\left(x_{L}<0\right)$. The total capacitance from contact to contact per unit area, $C_{t o t}$, is given by

$$
C_{t o t}=q \mid \frac{d}{d V} \int_{x_{L}}^{x_{R}}(n \text { or } p) d x \mid
$$

The location $x_{0}$ is the metallurgical junction and $x_{1}$ is the electrical junction which is the cross over point of the electron and hole concentration, i,e, $n\left(x_{1}\right)=p\left(x_{1}\right)$. Only for a symmetrical junction this electrical junction coincides with the metallurgical junction. Denoting the positions of the edges on the p-type and on the n-type side of the classical depletion region by $x_{p}$ and $x_{n}$ respectively, the total capacitance can be split into three components, as described by Sirsi and Boothroyd [11].

$$
C_{\text {tot }}=C_{s c r}+C_{\text {diff }}+C_{n}
$$


with

$$
{ }^{\cdot} C_{s c r}=q\left|\frac{d}{d V} \int_{x_{1}}^{x_{R}}(n-p) d x\right|
$$

$C_{s c r}$ is the space charge region capacitance that comes from the charge of the uncovered impurity doping atoms at the junction.

$$
C_{d i f f}=q\left|\frac{d}{d V}\left[\int_{x_{L}}^{x_{p}} n d x+\int_{\chi_{n}}^{x_{R}} p d x\right]\right|
$$

$C_{\text {diff }}$ is the diffusion capacitance stemming from the neutral charge (i,e, compensated carrier charges) in the quasi-neutral region of the device, and

$$
C_{n}=q\left|\frac{d}{d V}\left[\int_{x_{p}}^{x_{1}} n d x+\int_{x_{1}}^{x_{n}} p d x\right]\right|
$$

$C_{n}$ is the neutral capacitance associated with the storage of the neutral charge in the space charge region.

All the capacitances are per unit area. Each of the three components of the total capacitance has a different dependence on the applied voltage. 


\section{CHAPTER III}

\section{REVIEW OF EARLIER WORK ON THE SPACE CHARGE REGION CAPACITANCE}

In modeling the quasi static capacitance $C_{s c r}$ of the junction space charge region, an approach often used focuses on the change of the excess mobile carrier charges dQ in the space charge region per unit change of the voltage $\mathrm{dV}$. This approach leads us to the definition of Shockley [2]:

$$
C=\frac{d Q}{d V}=\frac{d}{d V} \int_{x_{n}}^{x_{q}} q(p \text { or } n) d x
$$

Eq. (3.1) in general, can be solved rigorously through the use of Eq.(2.1)-(2.3) provided that the boundaries $x_{n}$ and $x_{p}$, can be characterized as function of V. To simplify the discussion at this point, we will assume low level injection (minority carrier density is much smaller than the majority carrier density) prevails, and that the current density of the device is not high enough to cause any significant ohmic drop in the quasi-neutral region. Thus $V_{j}=V_{a p p}$, where $V_{a p p}$ is the applied voltage, $V_{j}$ is the voltage drop across the space charge region, which is commonly called the junction voltage.

In developing his theory Shockley [2], assumed that the space charge region of a $\mathrm{p}$ - $\mathrm{n}$ junction is essentially depleted of mobile charge carriers $(n=p=0)$. In addition he also assumed that the space charge region can be adequately approximated by an abrupt transition between entirely depleted semiconductor material and charge neutral semiconductor material. From this assumption Shockley [2], reasoned that $d V$ provokes an influx of hole charge, $q p\left(x_{p}\right) d x_{p}$, from the p-type side of the junction. From this concept he derived $C_{s c r}$ as, for the abrupt p-n junction; 


$$
C=\sqrt{\frac{q \varepsilon}{2\left(V_{b i}-V_{a p p}\right)} \cdot \frac{N_{A} N_{D}}{N_{A}+N_{D}}}=\frac{\varepsilon}{X}
$$

and for the linearly graded junction

$$
C=\left[\frac{q \varepsilon^{2} a}{12\left(V_{b i}-V_{a p p}\right)}\right]^{\frac{1}{3}}=\frac{\varepsilon}{X}
$$

where, $a$ is the junction gradient and $X=x_{n}-x_{p}$ is the thickness of the space charge region. Equations (3.1) and (3.2) are usually called the depletion model, which gives the space charge region thickness and capacitance as a function of $V_{a p p}$. The model is simple and compact, and the depletion approximation is adequate for reverse bias but introduces errors at forward bias.

A p-n junction SCR is composed of three regions: a depletion region and two partially screened regions. In the depletion region free mobile carriers are depleted and the impurity doping atoms are completely exposed. At the transition from quasi-neutral region to the depletion region there exists a partially screened region where free electrons and holes are partially depleted, hence the impurity doping atoms are partially exposed. The validity of the depletion approximation requires that the total charge in the depletion region from the exposed impurity doping atoms be much larger than that in the two partially screened regions. At reverse bias the depletion region is wide and the approximation is valid but at forward bias a significant amount of electrons and holes flow through the junction and the depletion region becomes narrower, so the depletion approximation accuracy decreases with increasing forward bias.

To improve the Shockley's depletion approximation model, Chang [6], studied the SCR capacitance for a step junction. Chang derived the space charge density by integrating Poisson's equation. Instead of focusing on free carrier charge, he defined $C_{s c r}$ by integrating the space charge density $\rho(x)$ in the space charge region as 


$$
C_{s c r}=\frac{d}{d V} \int_{0}^{\infty} \rho(x) d x
$$

Since the integrand vanishes beyond the depletion region the upper limit is irrelevant as long as it is larger than the depletion region. Chang considered the lower limit $x=0$, at the metallurgical junction. His calculation of capacitance showed good agreement with Shockley's model for the symmetrical abrupt junction with the difference of a minus $2 V_{t}$ correction of the $C_{s c r}^{-2} v s V_{a p p}$ intersection voltage. After the $2 V_{t}$ correction the classical depletion model becomes

$$
C=\sqrt{\frac{q \varepsilon}{2\left(V_{b i}-2 V_{t}-V_{a p p}\right)} \cdot \frac{N_{A} N_{D}}{N_{A}+N_{D}}}
$$

For asymmetrical abrupt junctions where $N_{A} \gg N_{D}$ or $N_{D} \gg N_{A}$, his capacitance calculations do not agree with Shockley's model nor do they agree with the experimental results of Muss [5]. This disagreement between Chang's and Shockley's space charge region capacitance model, increases as the asymmetry of the $p^{+-n}$ junction becomes more pronounced.

Kleinknecht [9], pointed out that the calculation of total space charge capacitance by Chang [6] was erroneous. For the important case where one side of the junction is much more heavily doped than the other side, mobile charges in the immediate neighborhood of the metallurgical junction are not negligible. These charges result form the carriers spilling over from the heavily doped side. The concentration of these carriers near the metallurgical junction may be large compared to the doping concentration on the lightly doped side. This situation has been corrected by Kleinknecht as follows. The total space charge density contributing to the SCR capacitance is not

$$
\int_{0}^{\infty} \rho(x) d x
$$

as used by Chang in Eq. (3.4), instead he calculated the total space charge in the junction, 
$Q$, from the electrical junction up to the contact, as

$$
Q=q N_{D} x_{1}+\int_{x_{1}}^{\infty} \rho(x) d x
$$

where, $x_{1}$ is the point of $p^{+-n}$ junction where the hole and the electron concentration are equal, which is the electrical junction, and $x=0$ is at the metallurgical junction. For asymmetrical abrupt doping profile the electrical junction does not coincide with the metallurgical junction. Kleinknecht calculated the built in potential for capacitance calculation as $V_{b i}=2 V_{t} \ln \left(N_{D} / n_{i}\right)$. He showed that the $C^{-2} v s V_{a p p}$ slope from his calculation has better agreement than the Shockley's model with the experimental values by Muss [5] for asymmetrical abrupt $p^{+-n}$ junction. The reason that Chang's model showed good agreement with Shockley's model for symmetrical abrupt doping profile, is that the electrical junction coincides with the metallurgical junction at $x=0$.

Gummel and Scharfetter [7] also suggested that if the contribution of the holes in the $p^{+}-n$ asymmetrical abrupt junction to the $\mathrm{n}$-type space charge region is considered when integrating $\rho(x)$, the resulting capacitance is inaccurate. In their calculation of capacitance they introduced a quantity $V_{x}$, called offset voltage, defined as the intercept of $C^{-2} v s V_{a p p}$, which is used in place of the built-in potential of the classical Shockley formula. They found that for symmetrical to low doping ratios $\left(\frac{N_{A}}{N_{D}}\right.$ or $\left.\frac{N_{D}}{N_{A}}<10\right)$ the offset voltage has the value of $V_{b i}-2 V_{t}$, independent of the applied voltage. For high doping ratios $\left(\frac{N_{A}}{N_{D}}\right.$ or $\left.\frac{N_{D}}{N_{A}}>100\right)$ the offset voltage is lower than $V_{b i}-2 V_{t}$, and is nearly independent of the doping ratio but increases nearly logarithmically with the applied voltage. The doping ratio at which the transition between the two regimes occurs depends on the applied voltage. The significance of the smaller offset voltage (intercept voltage) is that for forward and low reverse bias the capacitance is larger than that predicted by simple Shockley theory. 
Nuyts and Van Overstraeten [8] made computer calculation which justify the assumption that the quasi-Fermi potentials in the space charge region are almost spatially constant for the symmetrical and asymmetrical step junctions. Using the results of com. puter analysis Nuyts and Van Overstraeten developed an analytical method for calculating the junction capacitance. For symmetrical doping, their result was in complete agreement with earlier findings [6], [7], [9], and for asymmetrical doping profile the $C^{-2}$ vs $V_{a p p}$ intercept voltage is lower than the $V_{b i}-2 V_{t}$, and this difference increases with the increasing doping ratio. They also showed that this intercept voltage depends on the applied voltage. Their calculation method for intercept voltage is different from Gummel and Scharfetter [7], but ends up with the same conclusion.

Kennedy [4], studied the capacitance for a step junction for reverse bias and for thermal equilibrium. Kennedy derived an equation for the electric field in the space charge region by integrating the Poisson's equation. Like Nuyts and Van Overstraeten [8] and Kleinknecht [9] he also defined the space charge region capacitance of an abrupt $p^{+-} n$ junction as

$$
C=-q \frac{d}{d V_{a p p}} \int_{-\infty}^{x_{1}}\left[p(x)-N_{A}(x)+N_{D}(x)\right] d x+q N_{D} \frac{d x_{1}}{d V_{a p p}}
$$

Kennedy employed electrical field as the boundary condition for the integration. This method in general requires numerical solutions. But the method presents an analytical solution based on assuming a negligible accumulation layer for the symmetrical step junction, and by assuming that one type of the density of the mobile carriers greatly exceeds the density of the other type for an asymmetrical abrupt junction. For symmetrical abrupt junction his result is in complete agreement with several other authors [6]-[9]. For asymmetrical abrupt junction he showed that the depletion model Eq. $(3,4)$ produces increasing error with the increasing doping ratio. 
Figure 2, reproduced from Kennedy [4] shows that Eq. (3.4) produces a maximum

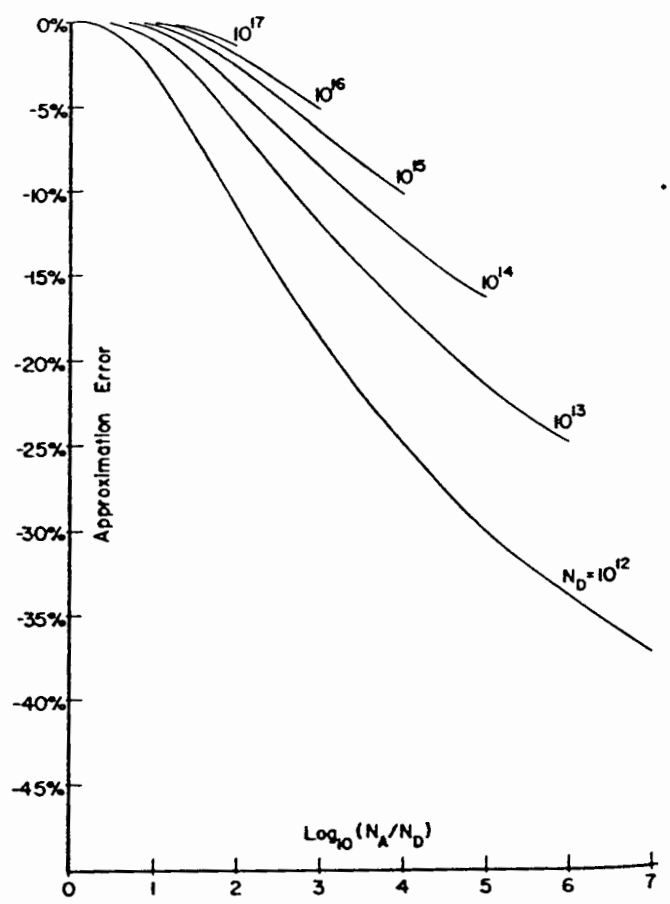

Figure 2.Percentage of error associated with Eq. (3.5) for asymmetrical abrupt junction. Reproduced from Kennedy [4], with written permission.

error of about $3 \%$ through out the doping ratio of $\left(1<\frac{N_{A}}{N_{D}}\right.$ or $\left.\frac{N_{D}}{N_{A}}<10\right)$ and an error of about $37 \%$ when $\frac{N_{A}}{N_{D}}=10^{7}$. Kennedy derived an analytical model for asymmetrical abrupt junction as in Eq. (3.9).

$$
C_{s c r}=\left[\frac{q \varepsilon N_{D}}{2\left(V_{b i}-V_{a p p}+V_{t}\left(1-\ln \frac{N_{A}}{N_{D}}\right)\right)}\right]^{\frac{1}{2}}
$$

which is valid for $\frac{N_{A}}{N_{D}}$ or $\frac{N_{D}}{N_{A}}>10$ gives a maximum error of about $3.5 \%$ at thermal equilibrium. He showed that his model for asymmetrical abrupt junction the $C^{-2} v s V_{a p p}$ intercept voltage depends only on the ratio of the doping concentration unlike the 
mel and Scharfetter [7] and Nuyts and Van Overstraeten [8] models. At this point Kennedy's model is more convenient to calculate the space charge region capacitance of an abrupt $p^{+-n}$ junction.

To improve the Shockley's model for linear graded junction and to accommodate forward voltages, Morgan and Smits [3], developed an expression for the total capacitance $C_{t o t}$,

$$
C_{t o t}=\frac{d}{d V} \int_{x_{L}}^{x_{R}} q p d x
$$

Using symmetry they decomposed Eq. (3.10) into a neutral capacitance $C_{n}$ and a space charge region capacitance $C_{s c r}$

$$
\begin{gathered}
C_{n}=\frac{d}{d V} \int_{0}^{x_{R}} 2 q p d x \\
C_{s c r}=\frac{d}{d V} \int_{0}^{x_{R}} \rho(x) d x
\end{gathered}
$$

In general $C_{t o t}=C_{s c r}+C_{d i f f}$, and $C_{s c r}$ cannot be explicitly obtained from MorganSmits model.

The physical reasoning underlying the separation of $C_{n}$ and $C_{s c r}$ is as follows. The free carriers will be depleted in the space charge region $(n=p=0)$ for reverse bias, which yields $C_{t o t}=C_{s c r}$, on the other hand, the space charge density $\rho(x)$ will approach zero if the junction is under high forward bias, because of the sizable density of the free carriers in the space charge region. This yields $C_{t o t}=C_{n}$. Thus the separation will enable one to visualize physically the meaning of $C_{t o t}$ at reverse and at high forward voltages. For intermediate voltages, however, the Morgan-Smits model requires numerical procedures, and introduces several auxiliary parameters. Morgan-Smits also derived approximate solutions for reverse bias and high forward voltages, which can be 
solved analytically.

The solution obtained by Smits and Morgan has limited applicability due to the assumption of no current flow in their analysis. In particular, this assumption is invalid at moderate or high forward applied voltage.

Chawla and Gummel [13] introduced an analytical model which is valid for reverse, zero, and small forward voltages. The model is valid for exponential-constant junction and its two extremes, the step and the linear graded junction. The model retains the same form as that of the Shockley's depletion capacitance model Eq.(3.2) and (3.3), but replaces $V_{b i}$ with an effective built-in voltage or offset voltage, so that the effect of free carrier charges in the space charge region is taken into account. Chawla and Gummel developed their model by first converting the Morgan and Smits [3] model into an asymptotic form for large reverse bias. They then compare the asymptotic form with the results of their contact-to-contact numerical solution. The numerical results agree with the asymptotic form except for the shallow junctions $\left(a<10^{14} \mathrm{~cm}^{-4}\right)$ and may be used for reverse bias up to small forward bias. Next they compared the asymptotic form with the expression for the depletion capacitance; the only difference between these two is the term corresponding to the built-in voltage.

From Chawla and Gummel it is seen that for linear graded junctions the transition region capacitance follows the $1 / C_{s c r}^{3}$ vs $V_{\text {app }}$ relation for reverse and low forward bias. $1 / C_{s c r}^{3} v s V_{a p p}$ intercept voltage is given by the offset voltage, which is a weak function of $V_{\text {app }}$. They introduced a new term "gradient voltage" $V_{g}$. This is dependent on the logarithm of the doping gradient and does not depend on the applied voltage.

$$
V_{g}=\frac{2}{3} V_{t} \ln \frac{a^{2} \varepsilon V_{t}}{8 q n_{i}^{3}}
$$


Chawla and Gummel justified that the gradient voltage is a good approximation of the offset voltage. Replacing the built-in potential with the gradient voltage, one should yield the SCR capacitance for linear graded junction. Their model is valid for reverse bias and up to a small forward bias.

Van Den Biesen [15],[16] decomposed the total capacitance $C_{t o t}$ into $C_{s c r}, C_{n}$ and $C_{\text {diff }}$. Instead of solving for $C_{s c r}$ numerically, Van Den Biesen solves $C_{s c r}$ asymptotically at the two extremes: reverse bias and very high forward bias. He used the numerical device simulator TRAP to confirm his analytical expression for the asymptotic values at high reverse bias and high forward bias, for symmetrical abrupt, asymmetrical abrupt and for linear graded junctions. For the symmetrical abrupt junction Van Den Biesen derived an analytical model which is applicable for any applied voltage. As the exact analytical solution for SCR capacitance is not possible for asymmetrical abrupt and linearly graded junction, he provided the solutions only for the two extremes: high forward bias and high reverse bias.

He showed that for the symmetrical abrupt doping profile, the maximum of the space charge region capacitance occurs at $V_{b i}-3.45 V_{t}$ and has a value of $0.243 \varepsilon / L_{D}$, where $L_{D}$ is the extrinsic Debye length. Comparison with the parallel plate capacitor equation $C=\frac{\varepsilon}{w}$ tells us that the minimum effective separation $w$ between the two capacitor plates in the SCR is approximately equal to $4 L_{D}$. Which leads us to consider that any potential variation in a semiconductor junction has a minimum spatial extension of a few Debye lengths. At that point the depletion approximation is bound to fail where $x_{n}$ is of the order of $L_{D}$. For linear graded junction he showed that the maximum value of $C_{s c r}$ will occur at $V_{b i}-3.4 V_{t}$ and has a value of $0.327 \varepsilon / L_{c}$, where $L_{c}$ is the characteristic length defined as 


$$
L_{c}=\left[\frac{3 \varepsilon V_{t}}{2 q a}\right]^{\frac{1}{3}}
$$

Van Den Biesen gives no details as to how the space charge region and quasi-neutral region capacitances are separated in the computer solution. He also assumed zero current densities for his device simulation program, which in fact is far from reality for high forward bias.

Van Halen [17] derived a new expression for $p-n$ junction space charge region capacitance keeping in mind not to introduce any new parameter for the SPICE circuit simulator. He described his model as

$$
C_{s c r}=\frac{C_{o}}{\left.1-\frac{V_{a p p}}{V_{b i}}+\frac{V_{t}}{2 V_{b i}} \exp \left(\frac{V_{a p p}-V_{b i}}{V_{t}}\right)\right]^{m}}
$$

This model reduces to Shockley's classical model Eq. (3.2) and (3.3) for reverse bias and for low forward voltages. At large forward voltages close, or above the built-in potential, exponential term dominates and the SCR capacitance decreases with an exponential dependence on $V_{a p p}$. The exponential decrease has been physically justified first by Sah [10] and later confirmed by Lindholm [12], [14], Van Den Biesen [15], [16].

Van Halen introduces the factor $\frac{V_{t}}{2 V_{b i}}$ before the exponential term in Eq. (3.15) as he justified that, if the applied voltage is equal to the built in voltage, where there is no potential barrier to the diffusion, the resulting capacitance is not zero, and can be compared to the flat band capacitance of an MOS capacitor, and thus for a one sided junction, at $V_{a p p}=V_{b i}$ Eq. (3.15) reduces to

$$
C_{s c r}=\frac{\varepsilon}{L_{D}}
$$


Van Halen's model for junction capacitance Eq. (3.15) is superior to the existing model in SPICE in a sense that his model uses only three SPICE input parameter, $V_{b i}$, $C_{o}$ and m, eliminating the parameter FC. In addition to that Van Halen's model also has a falling characteristics for a voltage above the built in potential.

Gummel and Poon [20] introduced a new model for SCR capacitance which is free of singularities, reaches to a peak, and then decreases at high forward bias. Their suggested model equation

$$
C_{s c r}=\frac{C_{o}}{\left(x^{2}+b\right)^{m / 2}}\left[1+\frac{m}{1-m} \frac{b}{x^{2}+b}\right]
$$

where $x=1-V_{a p p} / V_{b i}$ and $C_{o}, V_{b i}, m$ and $b$ are free parameters. These four parameters have to be determined from a fit of the experimental data. In developing their model, they deduced capacitance from the slope of a plot of emitter-to-collector delay time versus reciprocal emitter current. From that capacitance they obtained the best fit to those four parameters.

DeGraaff and Klaassen [21] derived a new expression for the charge in the space charge region, the derivative of which yields $C_{s c r}$. Their model is specifically meant for use in circuit simulation rather than physical justification. They described their space charge model as

$$
Q=\frac{1}{1-m} C_{o} V_{b i}\left[1-\left[\frac{1}{2}\left[\left(1-V_{a p p} / V_{b i}\right)+\sqrt{\left(1-V_{a p p} / V_{b i}\right)^{2}+K}\right]\right]^{1-m}\right]
$$

where $\mathrm{K}$ is a fitting parameter. This model requires four input parameters $V_{b i}, C_{o}, m$ and $K$ for the circuit simulator. Capacitance derived from this model keep $C_{s c r}$ finite at $V_{a p p}=V_{b i}$ and then reaches towards zero for $V_{a p p} \gg V_{b i}$. 


\section{CHAPTER IV}

\section{EXTRACTION OF CAPACITANCE FROM PISCES}

In this work the numerical device simulator PISCES-2B is used to obtain the exact value of the SCR capacitance. PISCES-2B can model the two dimensional distribution of potential and carrier concentration for any particular bias condition. It solves the Poisson's equation and both the electron and hole current continuity equations to analyze the devices.

Three partial differential equations describe most of the behavior of the semiconductor devices. Poisson's equation govern the potential distribution and hole and electron continuity equations govern the carrier concentrations. In order to solve these three equations for a device on a computer they must be discretized on a simulation grid. PISCES $-2 B$ solves these three partial differential equations for each of the grid point in the device. So, it solves for $3 \mathrm{~N}$ unknown real numbers, where $\mathrm{N}$ is number of nodes in the grid. Again, this discretization of the semiconductor device equations gives rise to a set of coupled nonlinear algebraic equations. There is no method to solve these equations in one direct step. PISCES-2B makes some initial guess and solves these equations by nonlinear iteration method.

A number of physical models are incorporated in the program for accurate simulations. Among those are, models for recombination, mobility, lifetime and bandgap narrowing. Both Boltzmann and Fermi-Dirac statistics, including the incomplete ionization are incorporated in PISCES-2B. It has the ability to attach the lumped resistive and capacitive elements to the contacts and to specify the distributed contact resistance. Both, 
voltage and current boundary conditions can be specified during a simulation. In addition, it is capable of performing an ac small-signal analysis which calculates the frequency dependent capacitances, conductances and admittances.

In order to extract the p-n junction space charge, it is necessary to know the electron and hole concentration distribution for the entire device. The electron and hole concentration versus distance for any particular applied voltage is extracted from PISCES. For the present work in PISCES simulations Boltzmann carrier statistics and complete ionization of the impurity doping concentration are used. Also the bandgap narrowing and concentration dependent mobility are taken into account and a temperature of $T=300^{\circ} \mathrm{K}$ is used in this simulation.

\section{IV.1 NET CHARGE METHOD}

For a p-n junction the space charge density is given by

$$
\rho(x)=q[p(x)-n(x)+N(x)]
$$

where, $\mathrm{N}(\mathrm{x})$ is the net ionized impurity doping concentration, which is positive for donor and negative for acceptor impurity atoms: $N(x)=N_{D}(x)-N_{A}(x)$.

Figure 3 shows the schematic diagram of a p-n junction device, which is used for simulation in PISCES. In Figure 4 holes $p(x)$, electrons $n(x)$ and net ionized impurity atoms $N(x)$ in absolute value are plotted versus distance for an applied voltage $V_{a p p}=0$, for a symmetrical abrupt junction shown in Figure 3 with doping concentrations $N_{D}=10^{17} \mathrm{~cm}^{-3}$ and $N_{A}=10^{17} \mathrm{~cm}^{-3}$. 


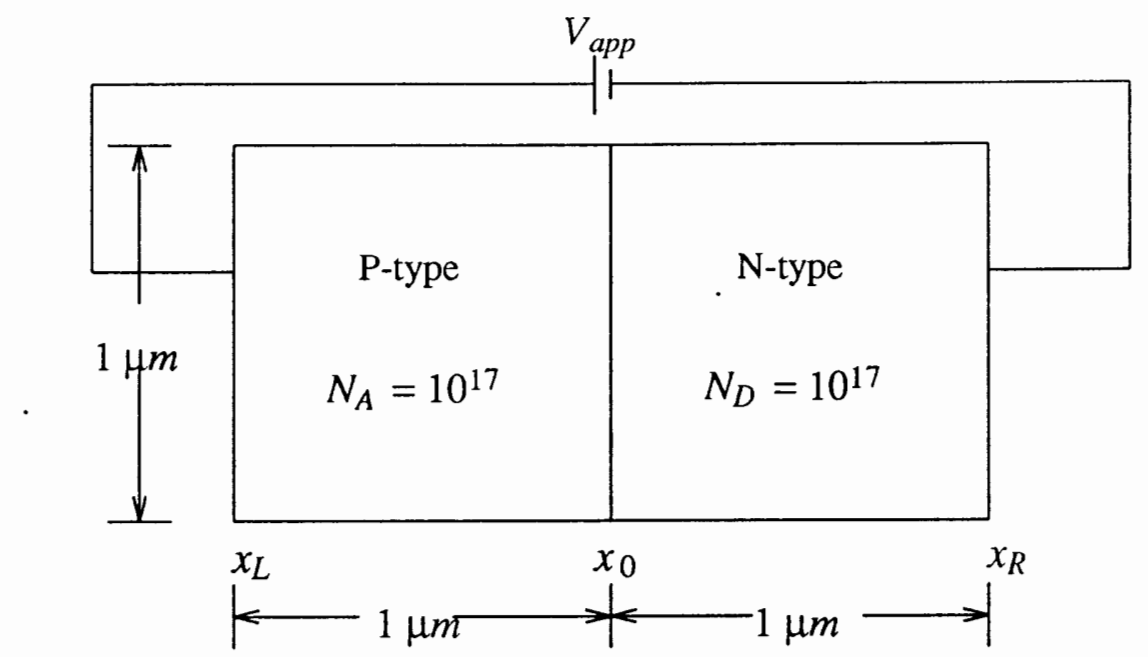

Figure 3.Schematic of $\mathrm{p}-\mathrm{n}$ junction device used for the device simulator PISCES.

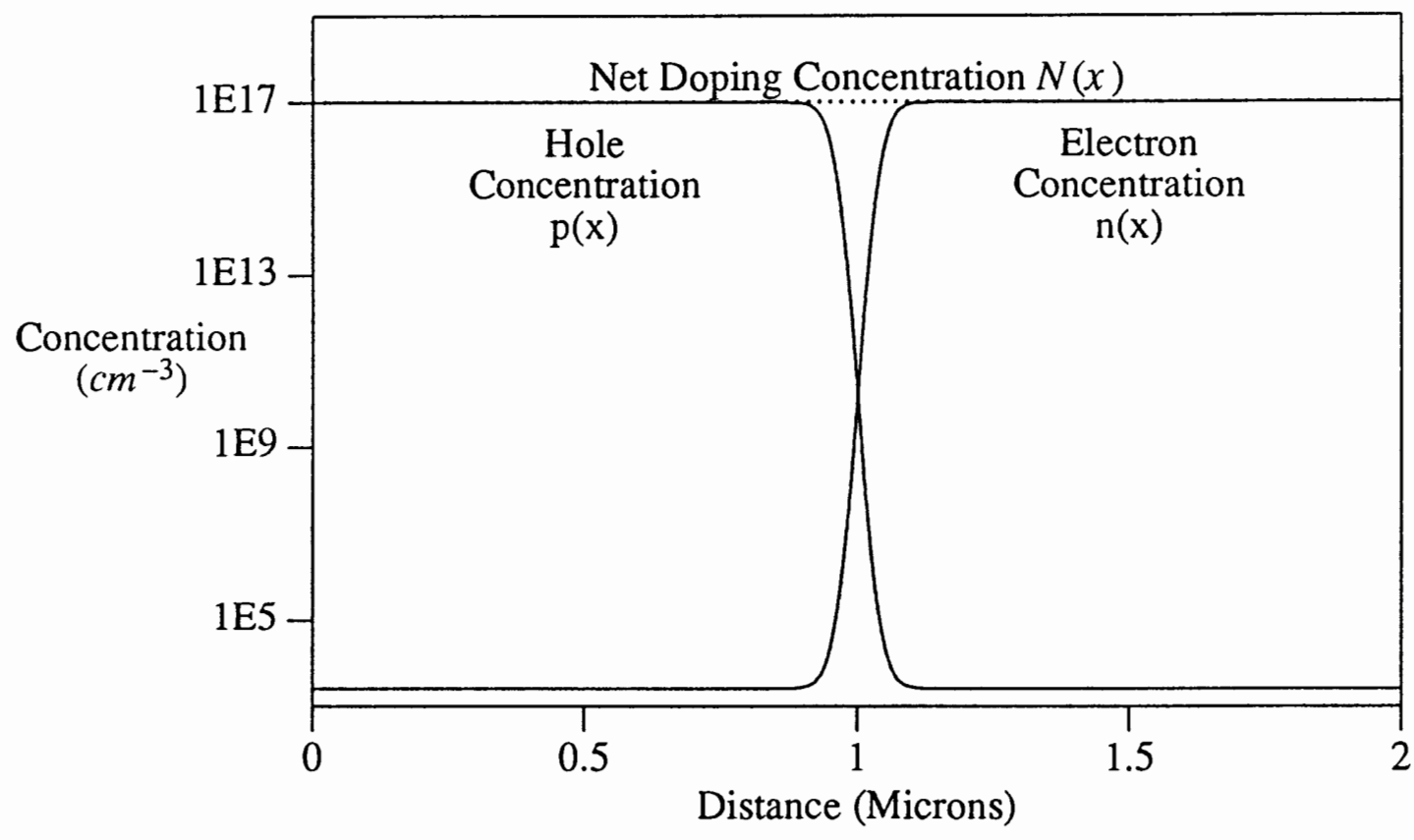

Figure 4.Distribution of electron and hole concentration for the device in Figure 3, extracted by PISCES for a symmetrical abrupt doping $N_{A}=N_{D}=10^{17} \mathrm{~cm}^{-3}$ and for applied voltage $V_{a p p}=0$.

Now the task at hand is to find the total space charge which contributes to the junction capacitance. PISCES solves the Poisson's and current continuity equations to compute the electron and hole concentration $n(x)$ and $p(x)$ for each point of the device. As 
complete ionization is used in the simulation model, $N_{A}=N_{A}^{+}$and $N_{D}=N_{D}^{+}$in each point. PISCES uses Eq. (4.1) to compute the net charge for each point of the device.

The net charge in number per cubic centimeter versus distance for applied bias $V_{a p p}=0$, is plotted in Figure 5, which shows that the depletion approximation is valid near the junction, $(n \approx p \approx 0)$, hence the net charge $\rho(x)$ is equal to the background doping concentration $N(x)$, but the tail at the edges of the space charge region shows the presence of the partially screened region which are only partially depleted.

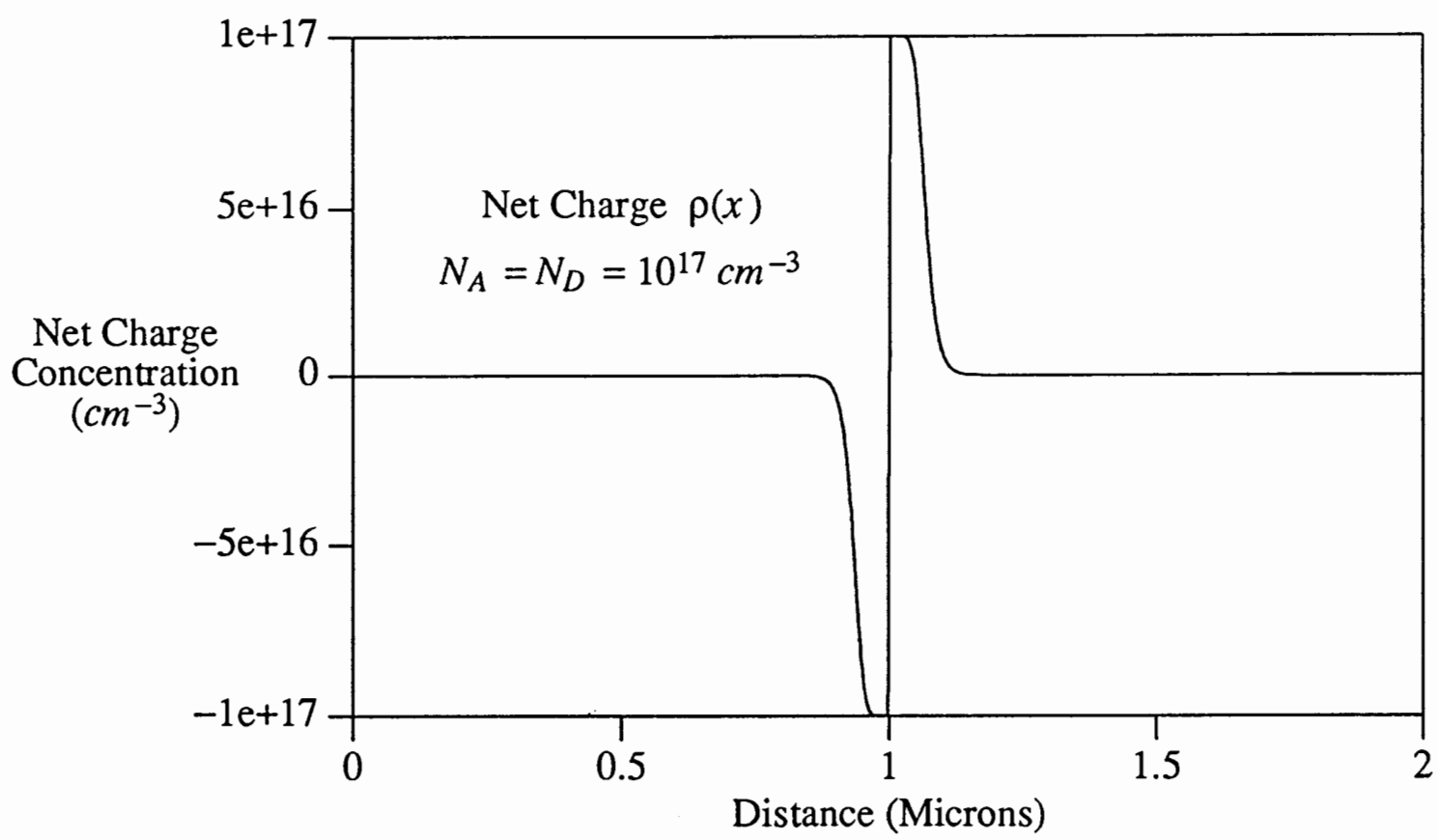

Figure 5.Space charge distribution $\left(\mathrm{cm}^{-3}\right), \rho(x)$, extracted by PISCES for the $\mathrm{p}-\mathrm{n}$ junction device shown in Figure 3.

The edge of the space charge region will be defined at the two points in the space charge region distribution, where the net charge concentration is about $1 \%$ of the peak net charge concentration. From these edges towards the junction is the space charge region and from these edges towards the contact is the quasi-neutral region. 
To get the total space charge in the $\mathrm{p}-\mathrm{n}$ junction one can integrate the net charge density $\rho(x)$. For the p-type side the net charge is negative, because $N(x)$ is negative. Similarly for n-type side the net charge density will be positive, because in Eq. (4.1) $N(x)$ is positive. Consequently integration of the net charge from contact to contact will result in zero, because the space charge in the p-n junction contributes the same amount of positive and negative charges. To get the total space charge that contribute to the $p-n$ junction capacitance one should integrate from outside the SCR to the electrical junction. For the device in Figure 3, or for any symmetrical doping profile, the electrical junction coincides with the metallurgical junction at $x=x_{0}$. When there is no variation of the net charge outside the SCR the integration limit can be set anywhere outside the SCR without any loss of accuracy. The result of integration of the net charge is shown in Figure 6.

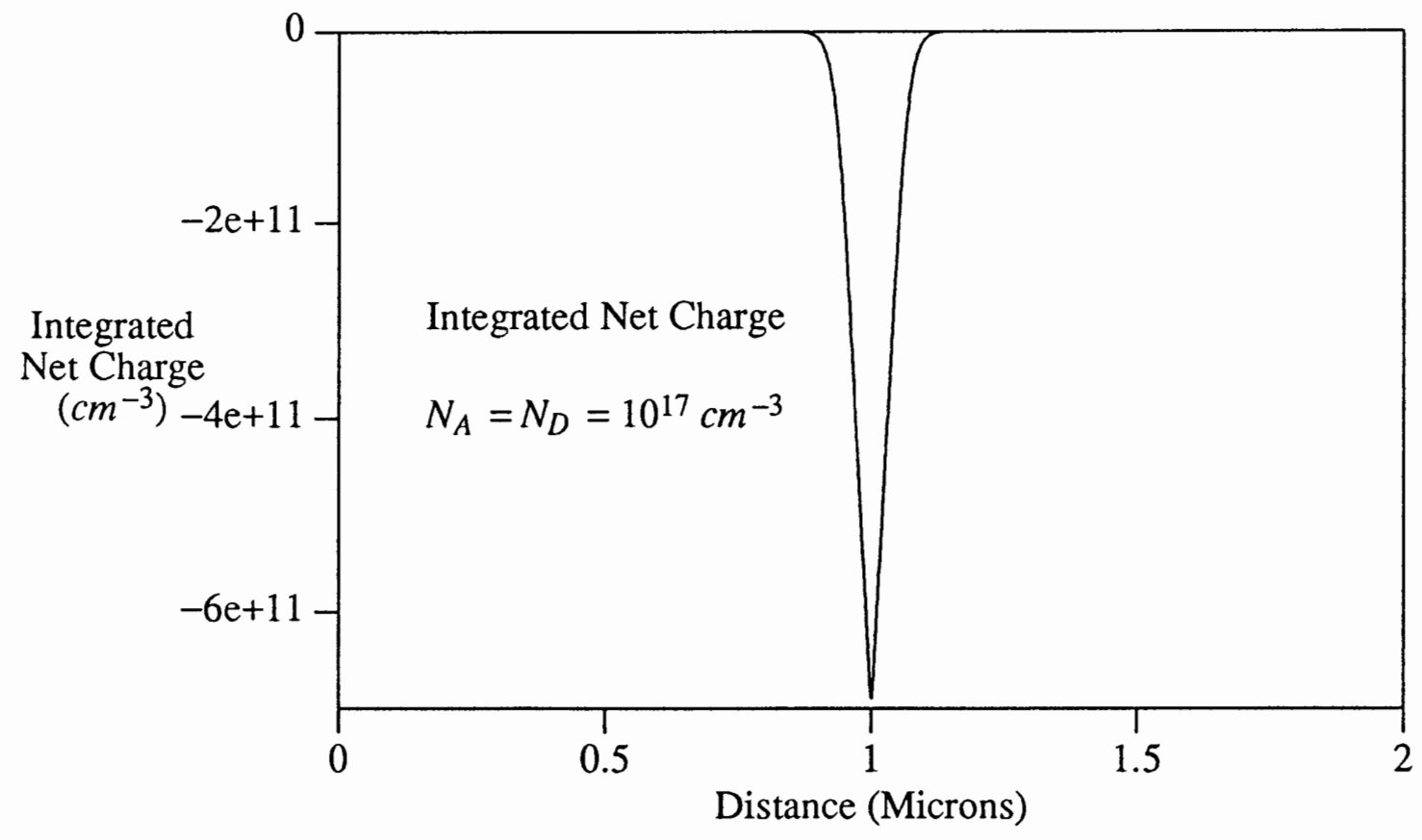

Figure 6.Integrated result of the space charge in Figure 5 from contact to contact for the junction shown in Figure 3. 
It is obvious that the integration of the net charge gives the electric field. Figure 6 also represents the electric field distribution in the structure after multiplying with some constants. From Figure 5 it is clear that the charge near the contact is zero and the presence of the net charge is observed when the transition region starts. Outside the transition region there is no net charge that contribute to the junction capacitance. Figure 6 integrates the net charge in Figure 5 and reaches a maximum at the electrical junction. Now this maximum point at the electrical junction corresponds to the total charge in one side of the transition region.

The SCR capacitance as defined in Eq. (3.1) is

$$
C_{s c r}=\frac{d Q}{d V_{a p p}}
$$

Now to get the SCR capacitance it is necessary to get the total charge in the space charge region for different applied voltages. If one can get the total charge for various applied voltages and the derivative of the total charge with respect to the applied voltage will yield the SCR capacitance. In PISCES the device in Figure 3 is solved for various applied voltages, starting from zero applied voltage and with an interval of $20 \mathrm{mV}$, up to a voltage far above the built-in potential. Then for each of this bias point the total charge in the space charge region is extracted by integrating the net charge from outside the SCR to the electrical junction. The total charge for each of these bias points is plotted in Figure 7. With the help of the circuit simulator Tekspice the derivative of $Q$, with respect to $V_{a p p}$ is taken and the SCR capacitance for various bias points is extracted.

Figure 8 shows the SCR capacitance with respect to applied voltage extracted from PISCES by net charge method. 


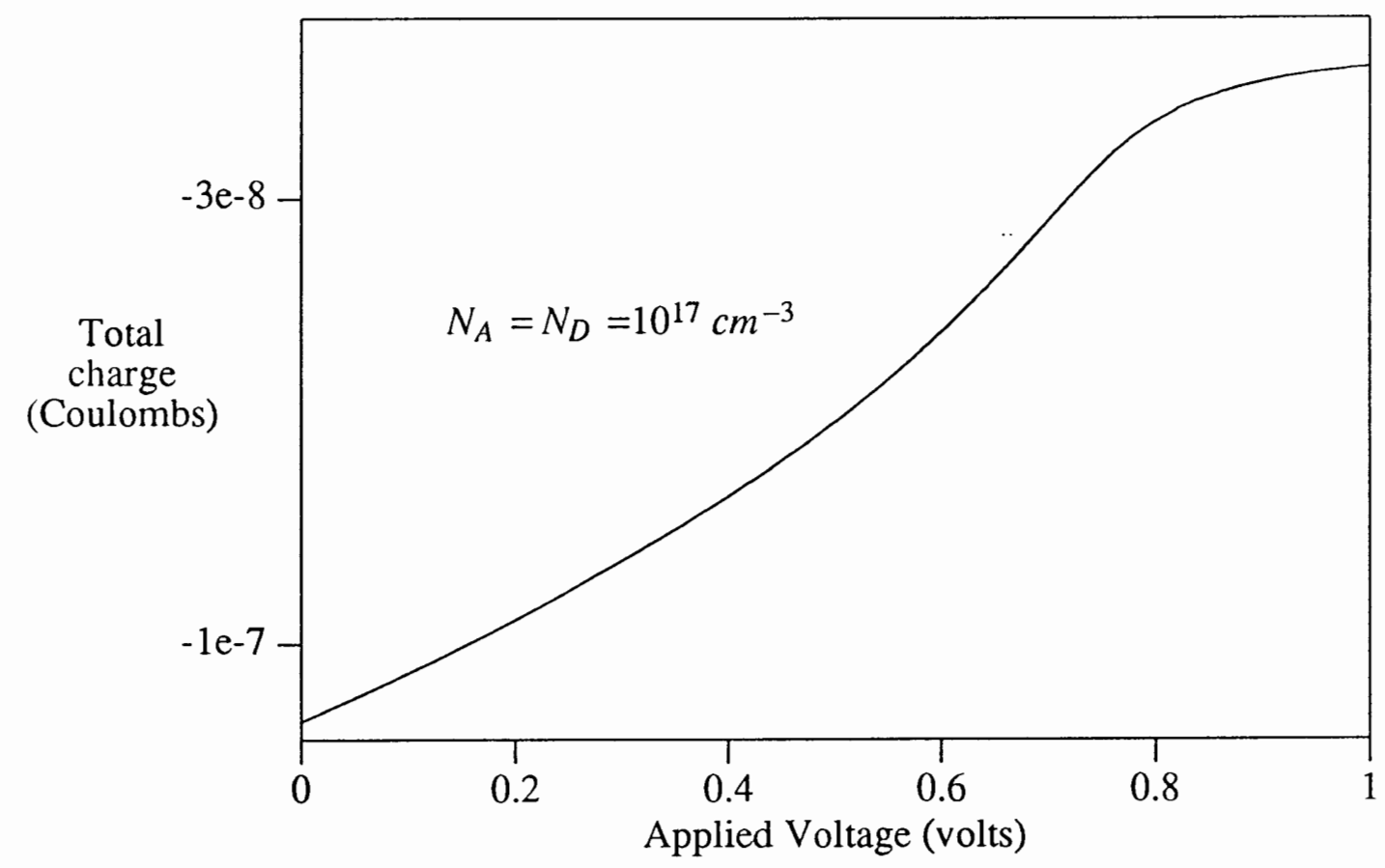

Figure 7.Total charge $\mathrm{Q}$ in the space charge region versus applied voltage for the device shown in Figure 3, extracted by PISCES.

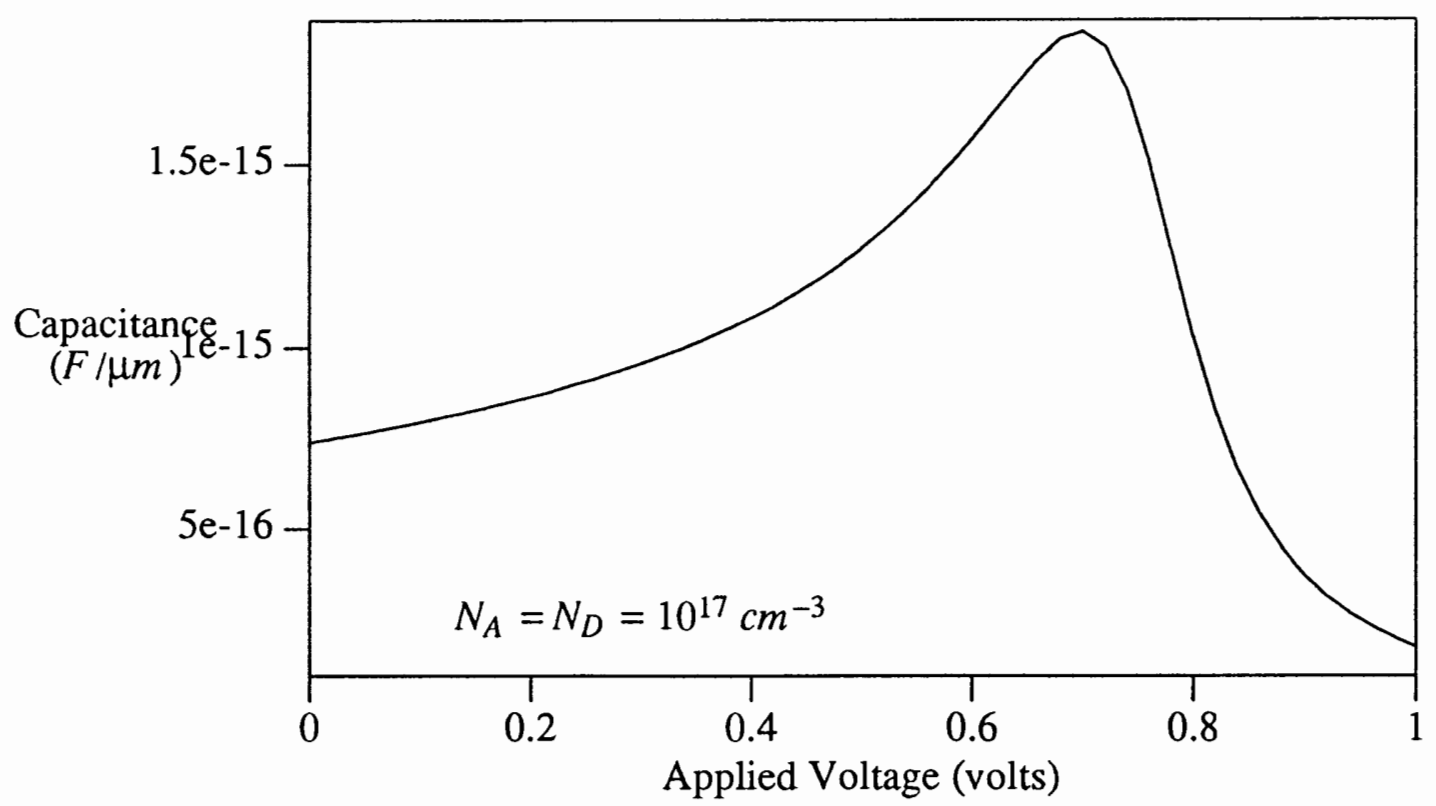

Figure 8.Derivative taken by Tekspice of the curve shown in Figure 7 with respect to the applied voltage, which is the space charge region capacitance. 
From Figure 9 it is evident that at high forward bias the charges accumulating near the contact will not be negligible. So the total charge from contact to the electrical junction is the sum of the charges from space charge region and the charges accumulated near the contact. Charges near the contact decrease with increasing forward bias but the charge in SCR of the p-n junction increases with increasing forward bias. PISCES takes the sum of these two charges to calculate the capacitance. To avoid integrating these charges near the contact in the net charge and the net carrier methods the lower integration limit was set just outside the space charge region.

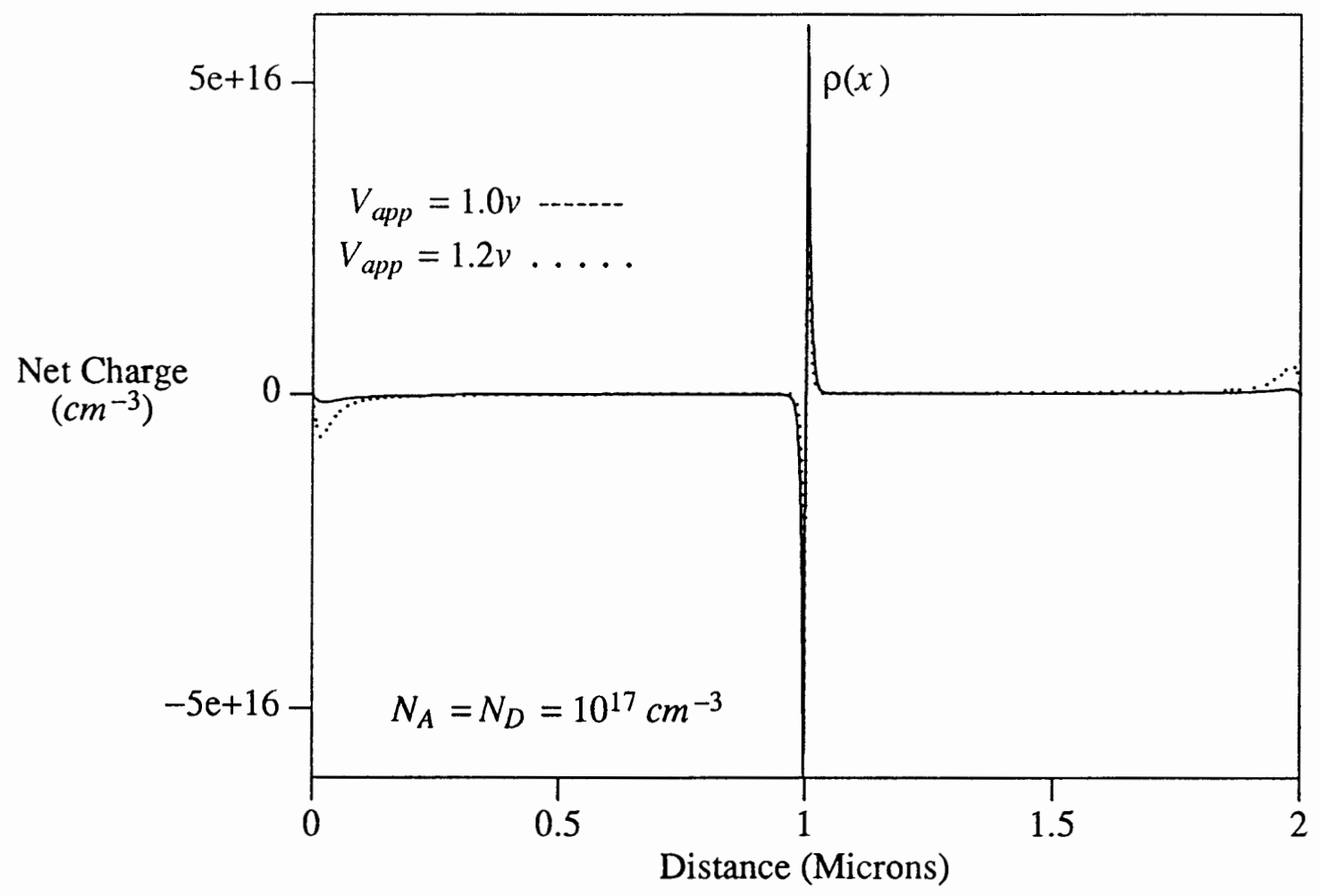

Figure 9.Space charge distribution, extracted from PISCES for the $p-n$ junction device shown in Figure 3.

To extract the SCR capacitance from the net charge method the following algorithm is used:

- Extract the net charge distribution for the entire structure from PISCES for the necessary bias points. 
- Integrate the net charge from outside the SCR to the electrical junction for each of these bias points.

- Take the peak value of the integrated net charge concentration, which is the total charge and plot it with respect to the applied bias.

- Take the derivative of the total charge with respect to the applied bias by Tekspice, which is the SCR capacitance.

\section{IV.2 NET CARRIER METHOD}

The second approach to extract the p-n junction capacitance from PISCES is by extracting the net carrier concentration. In Eq. (4.1) the first two terms of the left hand side, $p(x)$ and $n(x)$ change with the change of applied voltage, but the last term $N(x)$, the net doping concentration, does not change with the change of applied voltage. Hence, $p(x)-n(x)$ is just the net carrier concentration in the device. So it is possible to define the $p-n$ junction space charge region capacitance, as defined by Van den Biesen [15],

$$
C_{s c r}=q\left|\frac{d}{d V_{a p p}} \int_{x_{L}}^{x_{1}}(p(x)-n(x)) d x\right|
$$

It is possible to extract the net carrier distribution $p(x)-n(x)$ from PISCES.

Figure 10 shows that the net carrier concentration outside the transition region is equal to the background doping concentration, and decreases towards zero near the electrical junction. The net carrier concentration $p(x)-n(x)$ is positive in the p-type side and negative in the n-type side.

In order to find total net carrier concentration $\int_{x_{L}}^{x_{1}}(p(x)-n(x)) d x$ in one side of 
the $p-n$ junction, it is necessary to integrate the net carriers.

The integration of the net carriers is shown in Figure 10, which shows that the net carriers integrated from contact to contact results in zero, as $p(x)-n(x)$ in the p-type side is the same as that of n-type side with the opposite polarity. As the carriers are depleted in the vicinity of the electrical junction, the integrated net carrier shows a flat peak near the electrical junction. The peak at the junction in Figure 11 corresponds to the total net carrier in one side of the $p-n$ junction. As the change in the integrated net carrier corresponds to the SCR capacitance, there is no loss of accuracy to set the integration limit anywhere outside the space charge region, when there is no variation of the net carriers outside the SCR.

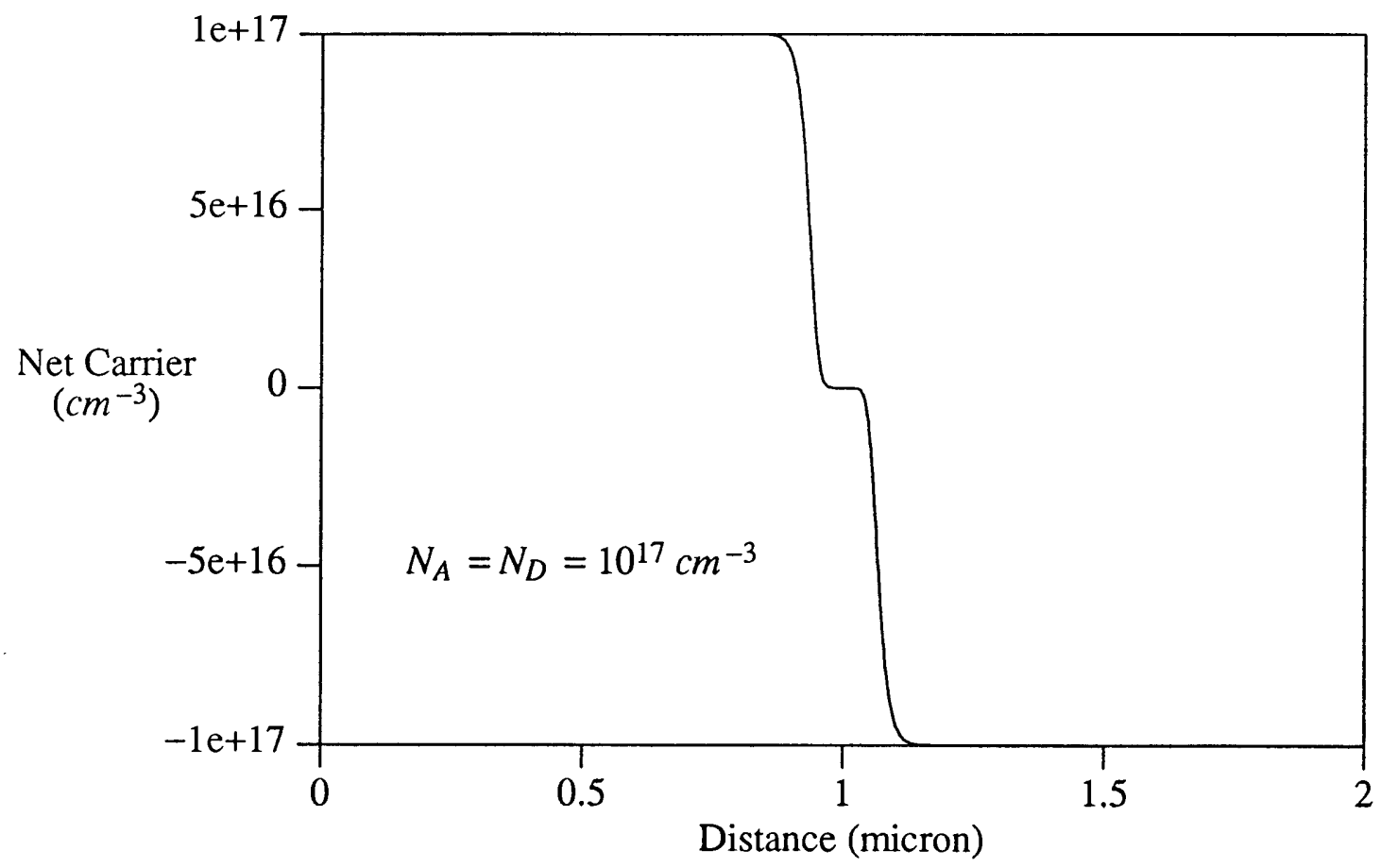

Figure 10.Distribution of net carrier concentration extracted by PISCES for $V_{a p p}=0$, for the device shown in Figure 3 . 


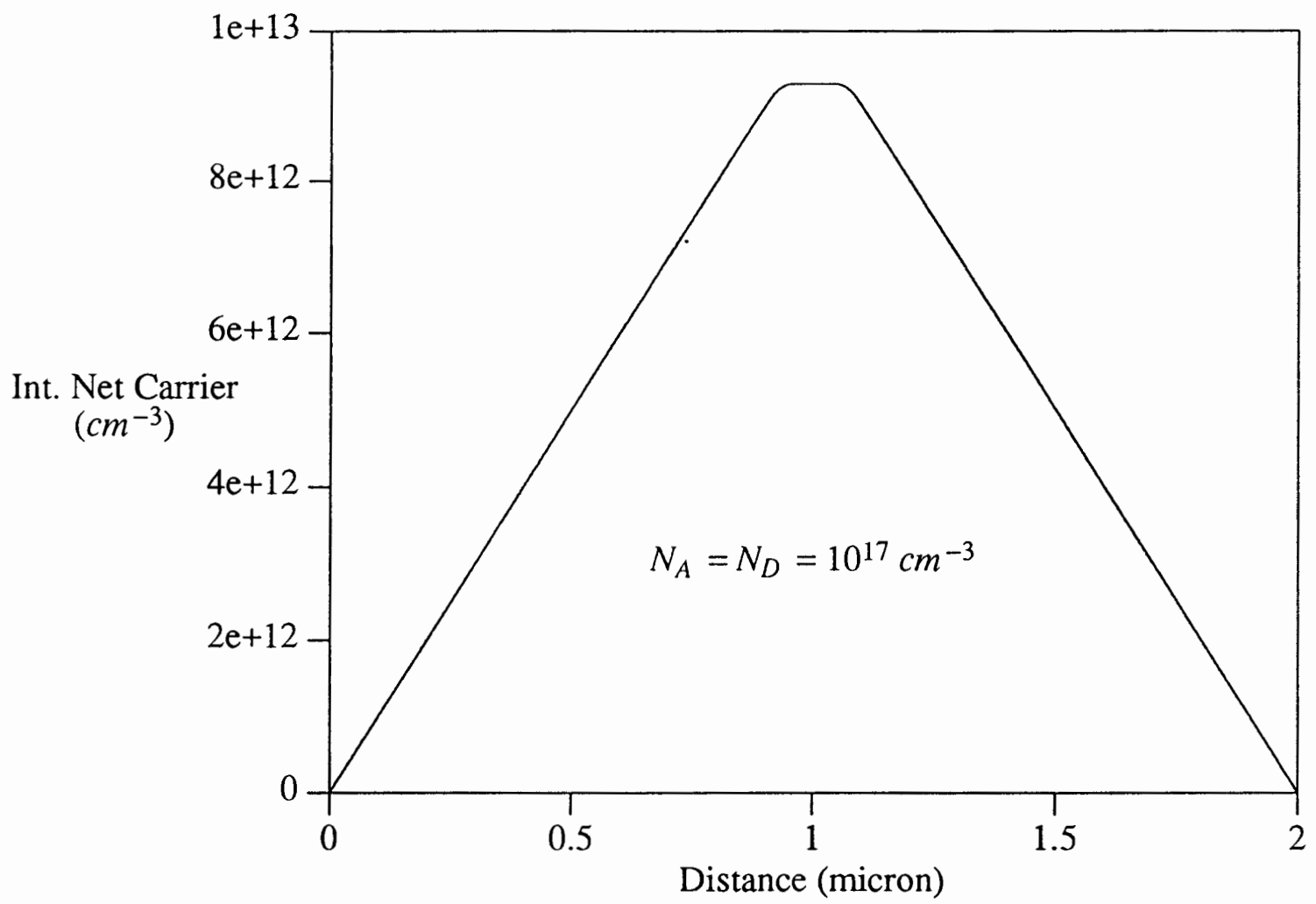

Figure 11.Integrated net carrier concentration extracted by PISCES for $\bar{V}_{a p p}=0$, for the device shown in Figure 3.

The Figure 12 shows that the integrated net carrier concentration per cubic centimeter in one side of the p-n junction for applied forward voltage from zero to one volt, with an interval of $20 \mathrm{mV}$. The integration limit was set just outside the SCR to avoid integrating the charges that accumulate near the contact at very high forward bias. As used in Eq. (4.3) the derivative of the integrated net carrier with respect to the applied voltage will yield the junction capacitance. In Figure 13 the extracted SCR capacitance from PISCES is plotted with respect to applied voltage. 


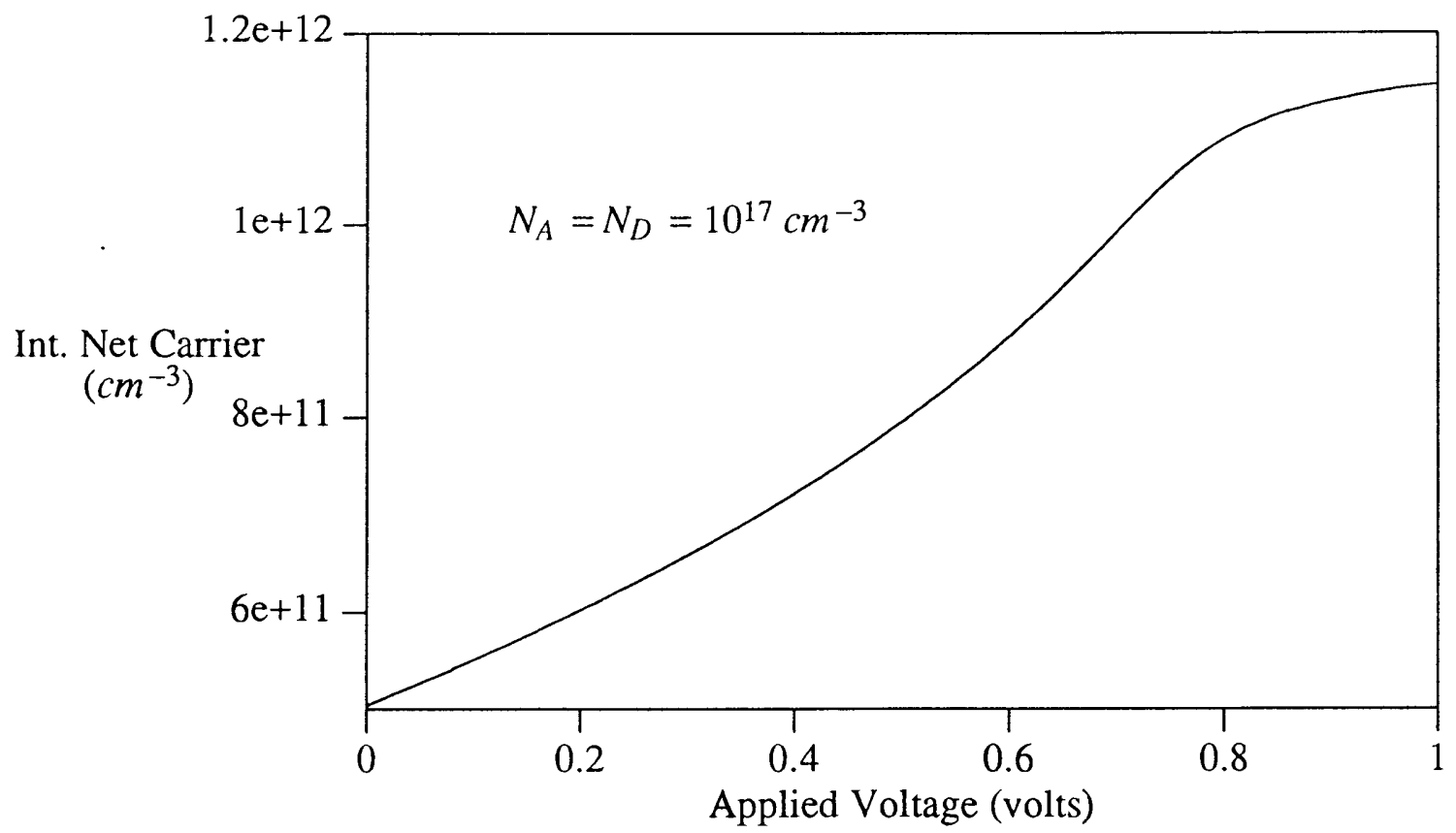

Figure 12.Integrated net carrier concentration extracted by PISCES, for the device shown in Figure 3. Integration limit is taken from 0.88 to 1.12 micron to avoid integrating the accumulated charge near the contact.

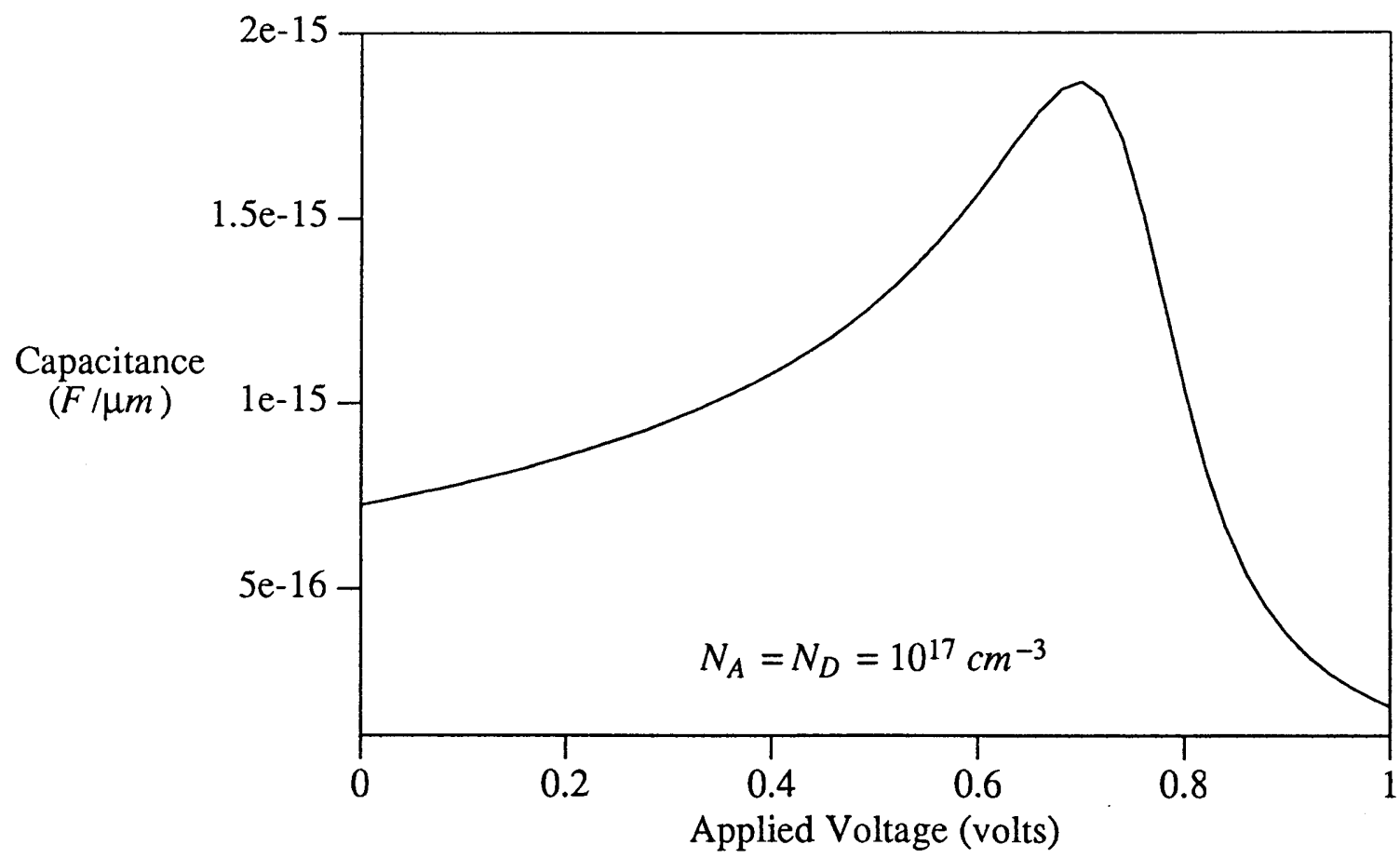

Figure 13.Derivative of the integrated net carrier concentration taken by Tekspice for the curve in Figure 12, which is the capacitance, $C_{s c r}$. 
To calculate the SCR capacitance from net carrier method the following algorithm is used.

- Extract the net carrier distribution for the entire structure by PISCES for the necessary bias points.

- Integrate the net carrier from just outside the SCR to the electrical junction for each of these bias points.

- Take the peak value of the integrated net carrier at the electrical junction and plot it with respect to the applied bias.

- Take the derivative of the integrated net carrier with respect to the applied bias, which is the SCR capacitance.

\section{IV.3 AC SMALL-SIGNAL ANALYSIS METHOD}

It is possible to calculate the capacitance from PISCES-2B directly. Starting from a dc bias condition, an input of given amplitude and frequency can be applied to the device structure from which PISCES calculates the terminal currents and voltages. Then using the relationship,

$$
Y_{i j}=\frac{I_{i}}{V_{j}}=G_{i j}+j \omega C_{i j}
$$

the frequency dependent admittance matrix, and hence the capacitance can be calculated.

In ac small-signal analysis method, PISCES calculates the capacitance from contact to contact, which includes both the diffusion and the space charge region capacitance. PISCES can not differentiate between the diffusion and space charge region capacitance. The capacitance shown in Figure 14 by ac small-signal analysis is the sum of the diffusion and the space charge region capacitance. 


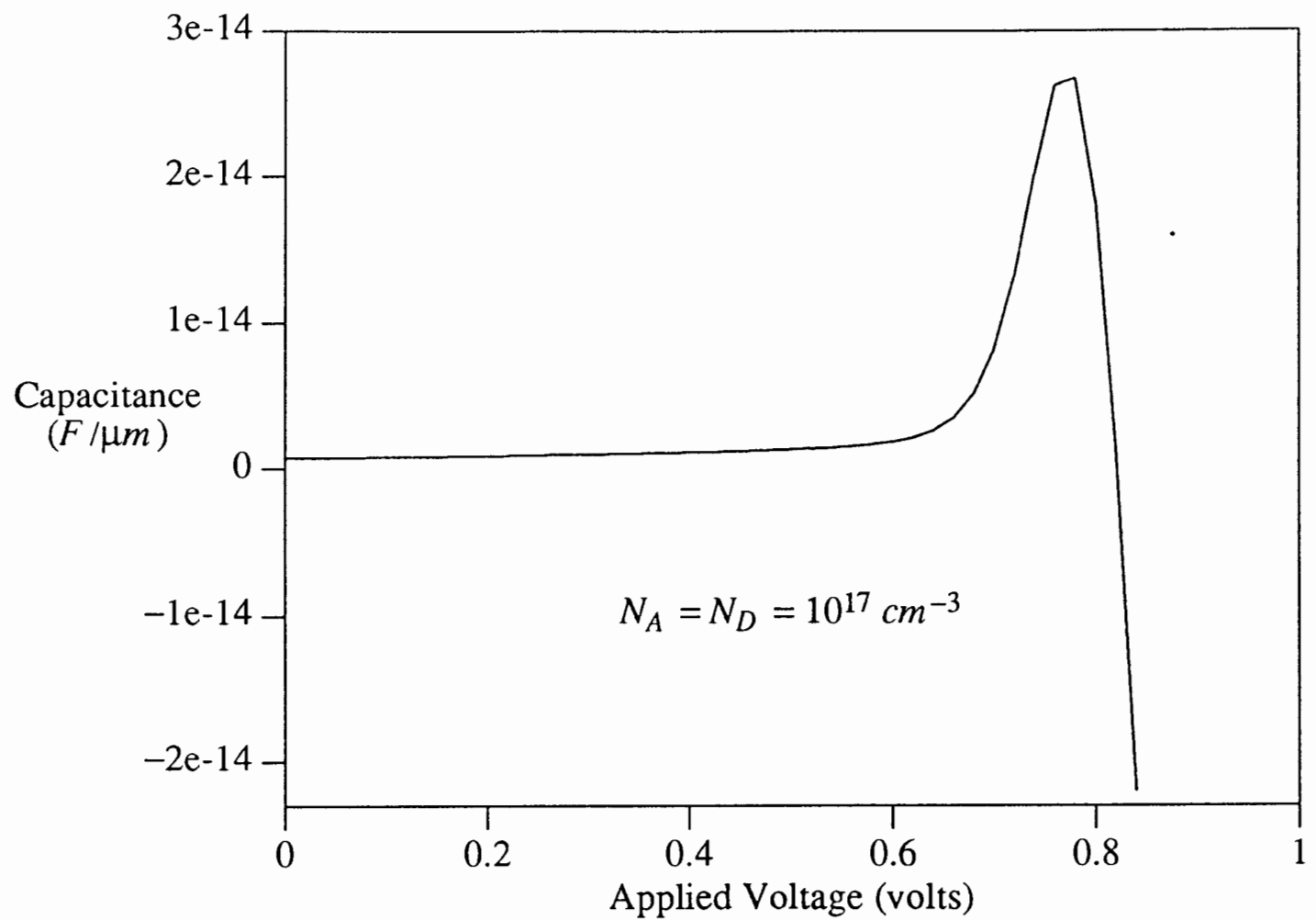

Figure 14.Capacitance extracted by PISCES from ac small-signal analysis.

It is also observed from the Figure 14 that at very high forward bias PISCES is giving a negative capacitance, i.e. the device behavior becomes inductive. This agrees with the findings of Van Den Biesen [19], who has shown that the ac capacitance of a p-n diode will change its sign from positive to negative when the total series resistance of the diode becomes equal to its differential resistance. Figure 15 shows that the capacitance extracted by ac small-signal analysis method is the same as net charge method for lower forward voltage, where the space charge region capacitance dominates the diffusion capacitance, but differs by orders of magnitude at high forward bias where the diffusion capacitance starts to dominate. So the ac small-small signal analysis method in PISCES can not be used to extract SCR capacitance. 


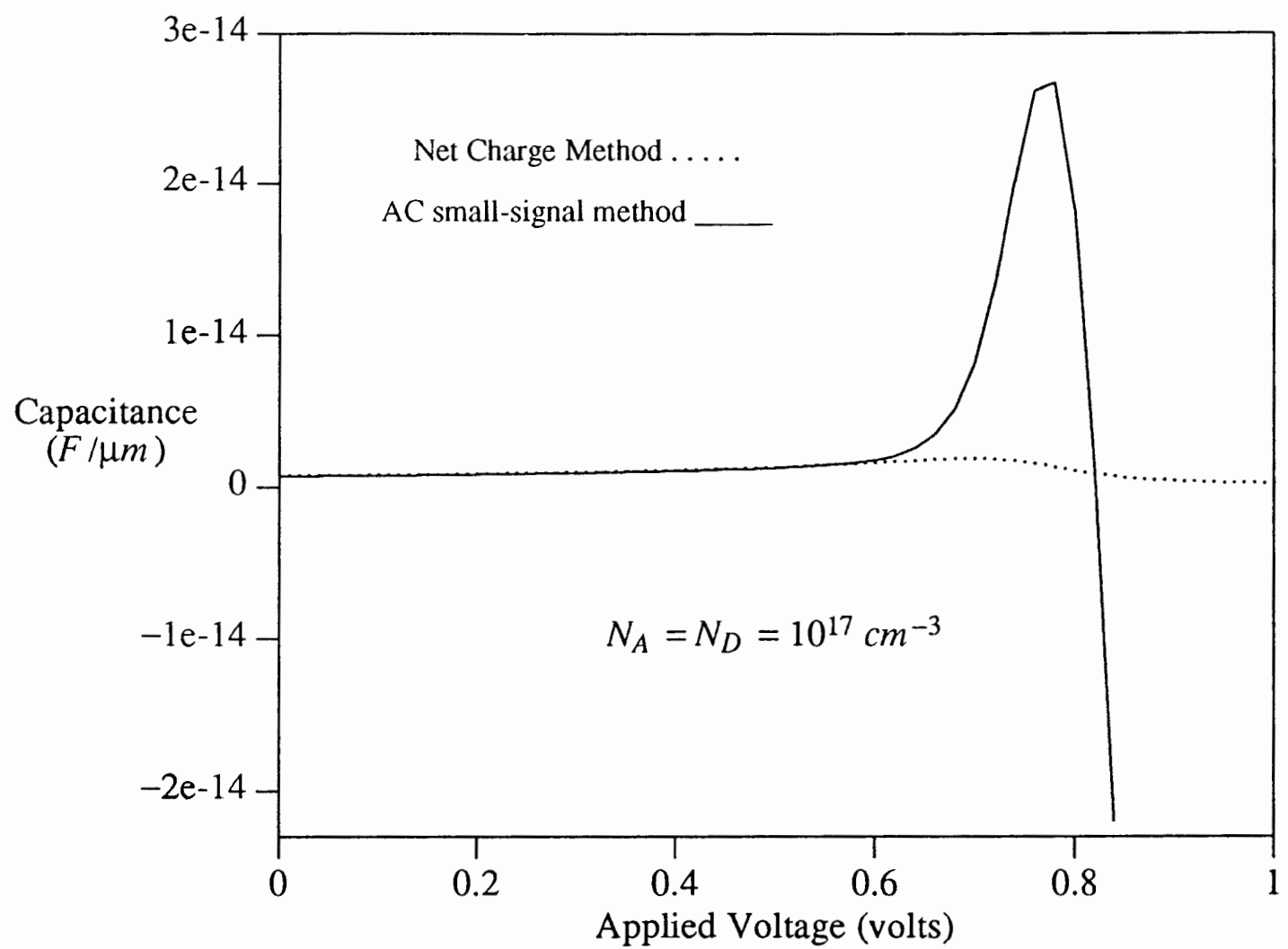

Figure 15.Comparison of capacitances extracted from PISCES by ac small-signal analysis and by net charge method.

\section{IV.4 CAPACITANCE VS QUASI-FERMI SEPARATION}

Until now to extract the p-n junction capacitance Eq. (4.2) has been used where the p-n junction space charge was considered to vary with respect to applied voltage between the two terminals. This assumption is valid for reverse bias and for low forward bias, but introduces errors for high forward bias, where part of the applied voltage is absorbed in the quasi-neutral region as an ohmic drop. So, a new variable, $\phi_{S C R}$, the separation between the hole quasi-Fermi level, $\phi_{p}$, and the electron quasi-Fermi level, $\phi_{n}$, at the space charge region, will be introduced here and the space charge region capacitance will be defined as,

$$
C_{s c r}=\frac{d Q}{d \phi_{S C R}}
$$


As both the majority and minority carrier concentration have to be at thermalequilibrium concentration at the contact, the electron quasi-Fermi level and the hole quasi-Fermi level have to be the same at the contacts. The difference of the quasi-Fermi levels between the n-type side contact and the p-type side contact is equal to the apllied voltage between the two terminals.

Figure 16 shows the spatial distribution of the electron and hole quasi-Fermi level, for $V_{a p p}=0.3$ volts. It shows that $\phi_{p}$ is constant from the p-region contact up to and through the junction, similarly, for the n-type side $\phi_{n}$ is constant from the n-type side contact up to and through the junction. The difference of the quasi-Fermi levels between the p-region contact and the n-region contact is equal to 0.3 volts which is equal to the applied voltage.

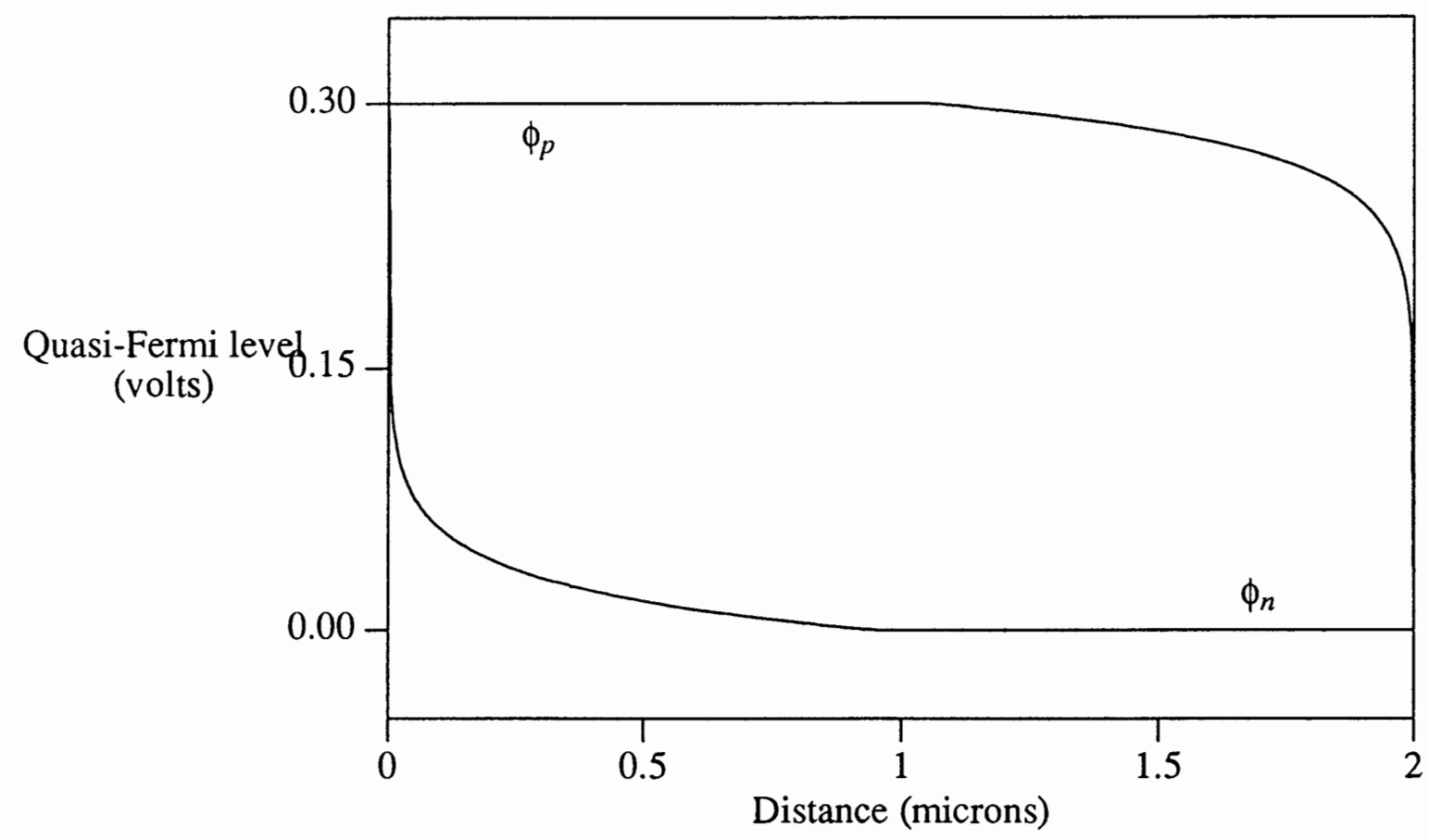

Figure 16.Distribution of hole and electron quasi-Fermi level for $V_{\text {app }}=0.3$ volts, extracted from PISCES. 
Van Vliet [1] and Toshoi Misawa [18] pointed out that the quasi-Fermi level drop in the quasi-neutral region is nothing but the ohmic drop. So the quasi-Fermi level separation between the contacts is the sum of the quasi-Fermi level separation at the space charge region and the ohmic drop in the quasi-neutral region. Quantitatively,

$$
V_{a p p}=I\left(R_{n}+R_{p}\right)+\phi S C R
$$

where, $I$ is the current through the structure, $R_{n}$ is the resistance of the n-type quasineutral region, $R_{p}$ is the resistance of the p-type quasi-neutral region. At reverse and low forward bias the current density in the device is not high enough to cause any significant potential drop in the quasi-neutral region (QNR) of the device, so quasi-Fermi levels, $\phi_{p}$, in the p-type side and $\phi_{n}$, in the n-type side are constant and the applied voltage across the terminal is equal to the quasi-Fermi level separation across the SCR.

At high forward voltages the current in the device is sufficient to cause a significant bending of the quasi-Fermi levels. Chawla and Gummel [13] showed that at high forward bias the assumption of constant quasi-Fermi level throughout the SCR does not introduce any significant error in calculating the $C_{s c r}$. They have verified this assumption by comparing results with those obtained by a more accurate analysis which takes into account the bending of the quasi-Fermi levels. So for forward bias it is reasonable to assume that $\phi_{s c r}$ is equal to $\phi_{p}-\phi_{n}$ at the metallurgical junction. Figure 17 shows the distribution of electron and hole quasi-Fermi level for $V_{a p p}=1.0$ volts.

Figure 18 shows the variation of the $\phi_{S C R}$ versus the applied voltage. At lower voltage $\phi_{S C R}$ is equal to $V_{a p p}$ and the curve is a straight line with unity slope. At higher forward bias where the ohmic drop in the quasi-neutral region starts to take place, $\phi_{S C R}$ starts to deviate from $V_{a p p}$. In order to find the p-n junction capacitance trough the definition of Eq. (4.5), it is necessary to get the total space charge with respect to the quasi-Fermi level separation. 


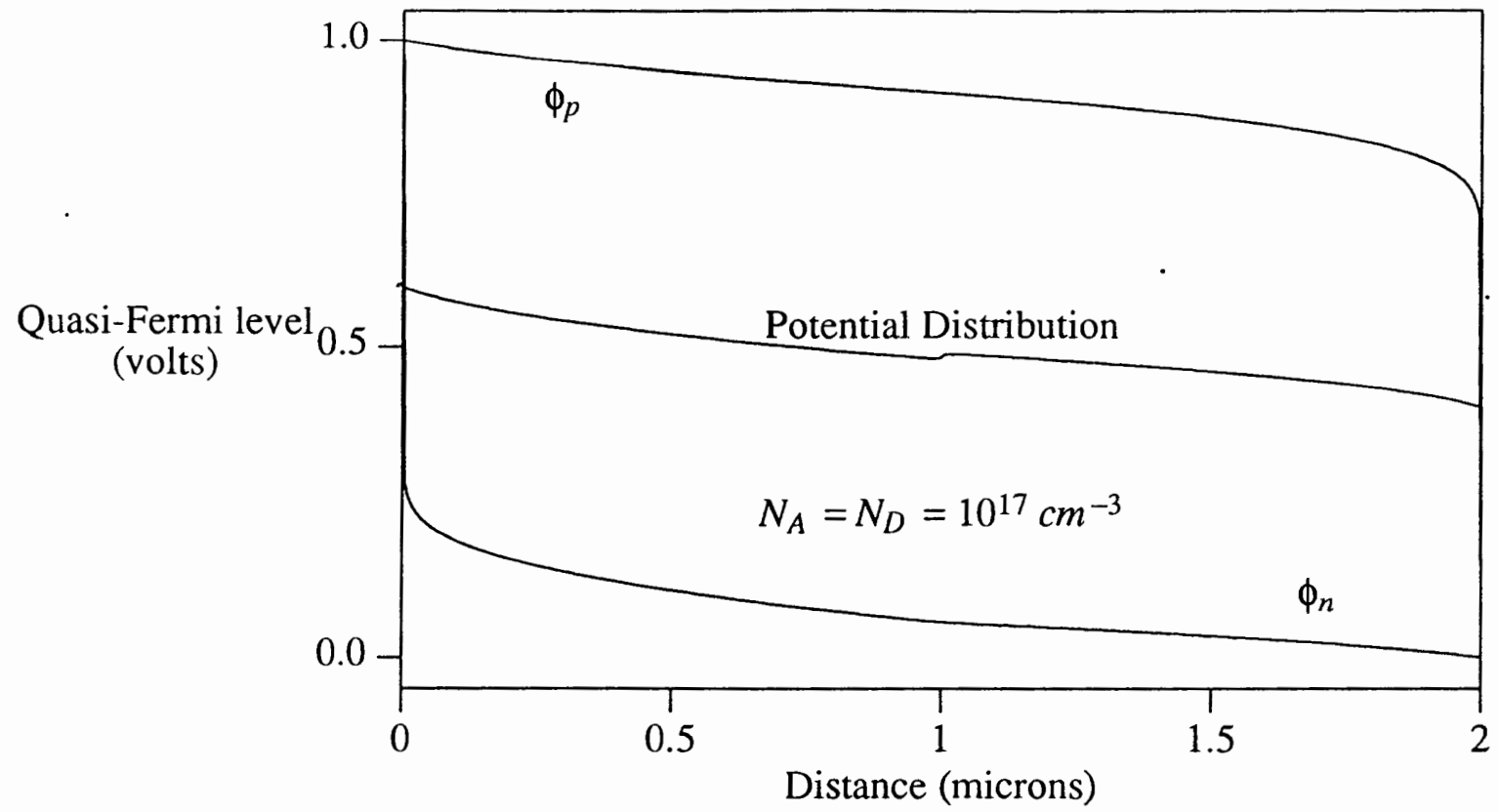

Figure 17.Distribution of hole and electron quasi-Fermi level and potential for $V_{a p p}=1.0$ volts. Extracted from PISCES.

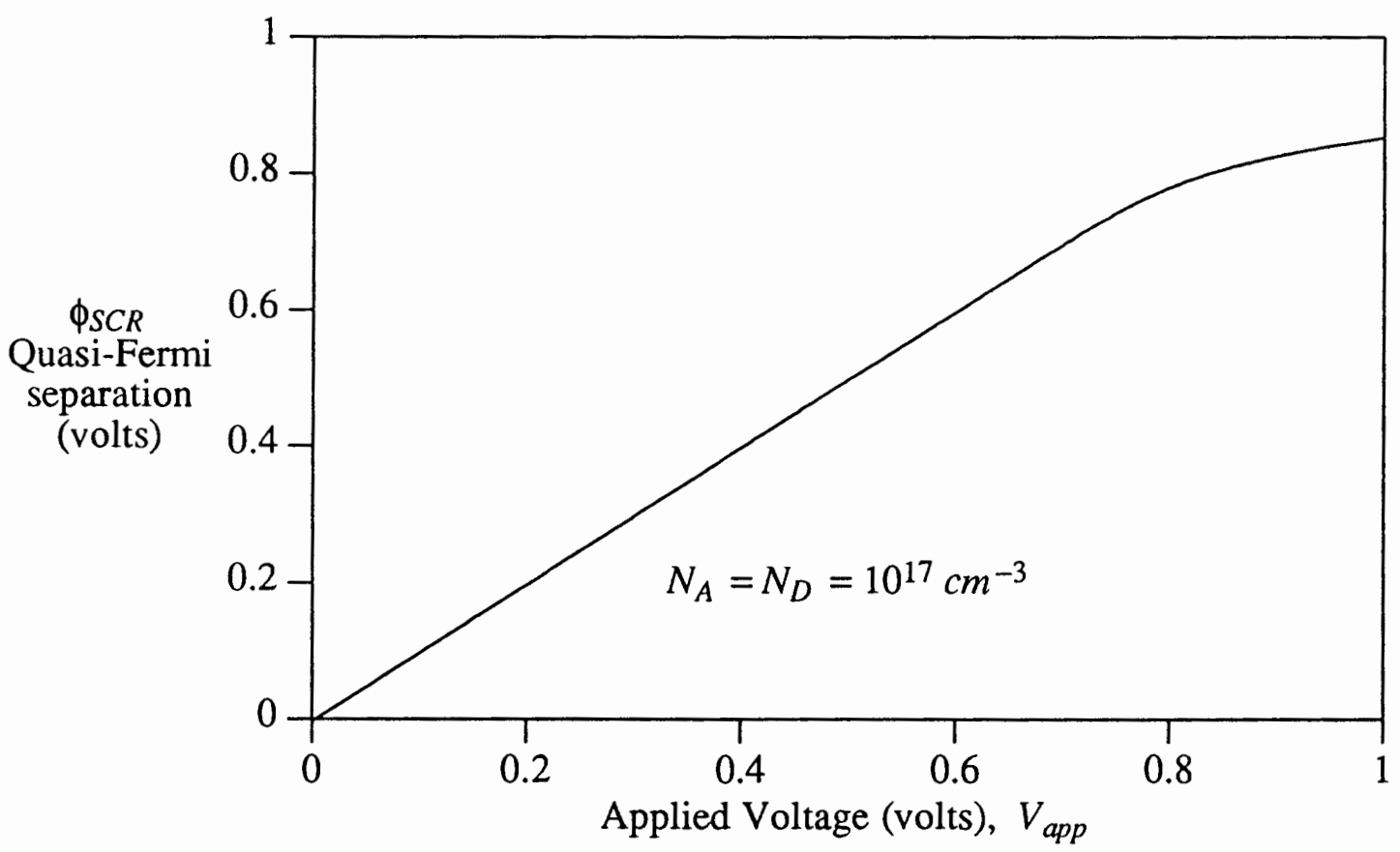

Figure 18.Quasi-Fermi level separation at the junction $\phi_{S C R}$ versus

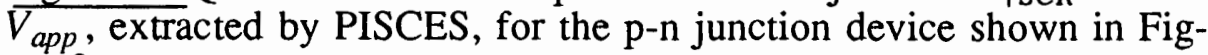
ure 3. 
In Figure 19, the total charge in the space charge region is plotted against the quasi-Fermi level separation. By Tekspice the derivative of $\mathrm{Q}$ with respect to $\phi_{S C R}$ is taken and the result of which is the SCR capacitance.

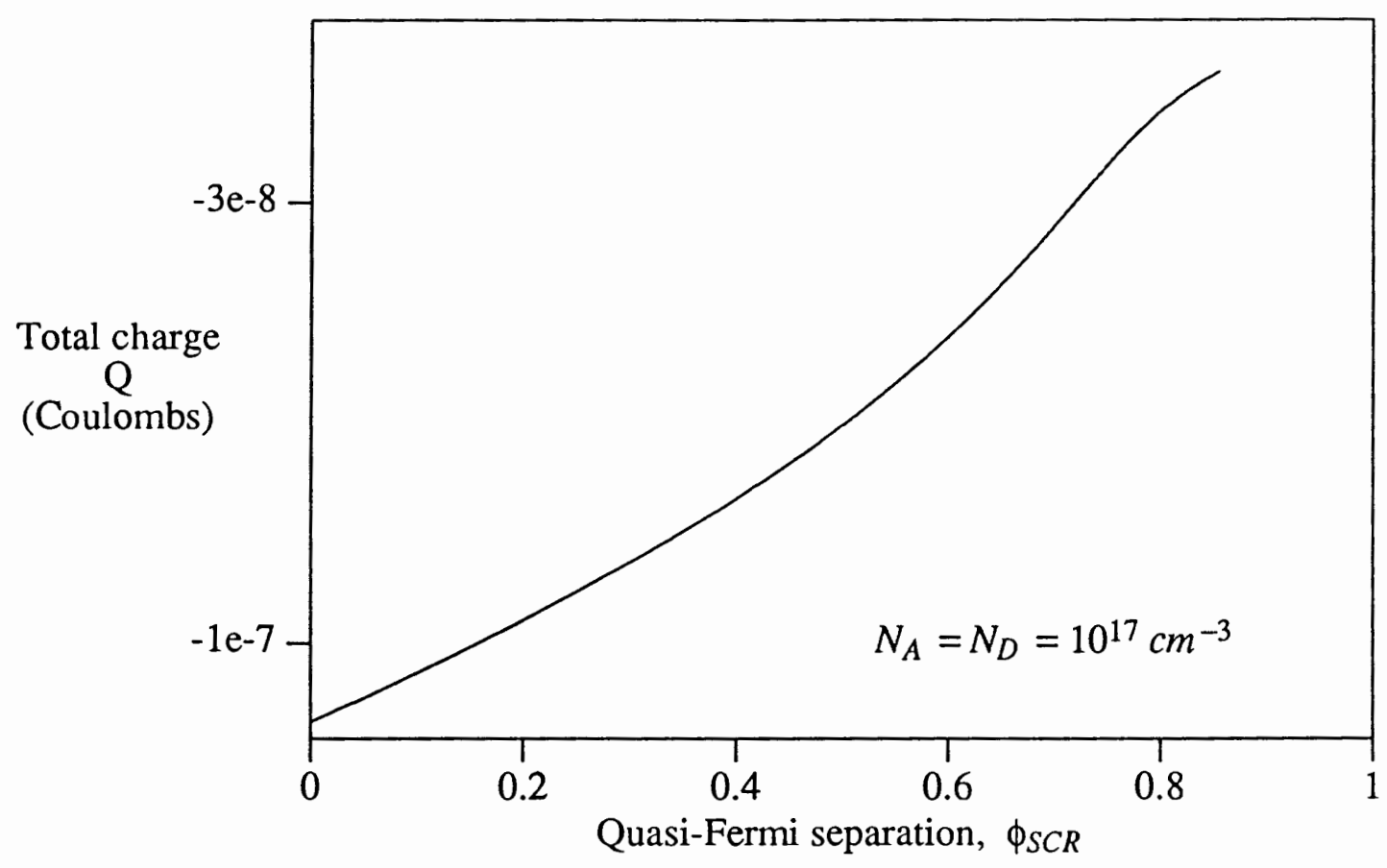

Figure 19.Total space charge $Q$ versus quasi-Fermi separation. Extracted by PISCES for the p-n junction device shown in Figure 3.

Figure 20 shows that the capacitance with respect to quasi-Fermi level separation follows a $\sqrt{\phi_{S C R}}$ relation for zero to lower forward voltages and decreases exponentially at high forward voltages.

The peak of $C_{s c r}$ extracted by PISCES is enlarged in Figure 21. The value of the peak is approx. $1.95 \mathrm{fF} / \mu \mathrm{m}$ which agrees with $0.243 \varepsilon / L_{D} \approx 1.95 \mathrm{fF} / \mu \mathrm{m}$ and the location of the peak is at $\phi_{s c r}=0.718$ volts, which agrees with $V_{b i}-3.45 V_{t} \approx 0.718$ volts. To compare the location and value of the peak the potential step for simulation in PISCES was $5 \mathrm{mV}$. Thus the capacitance extracted from PISCES is verified for its validity with the earlier findings of Van Den Biesen [15]. 


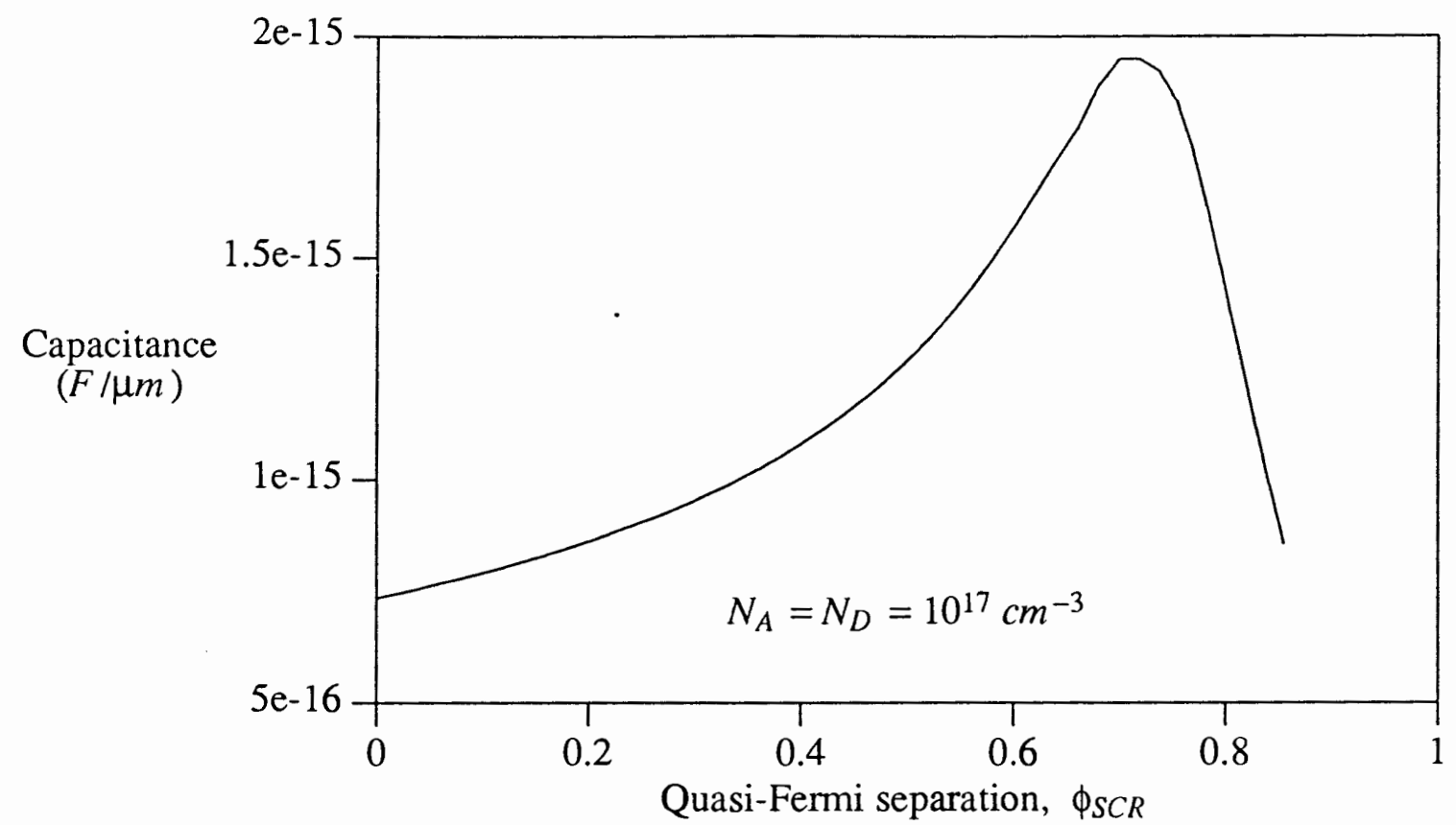

Figure 20.Derivative of the total space charge with respect to quasiFermi level separation, taken by Tekspice for the curve in Figure 19. Which is the space charge region capacitance.

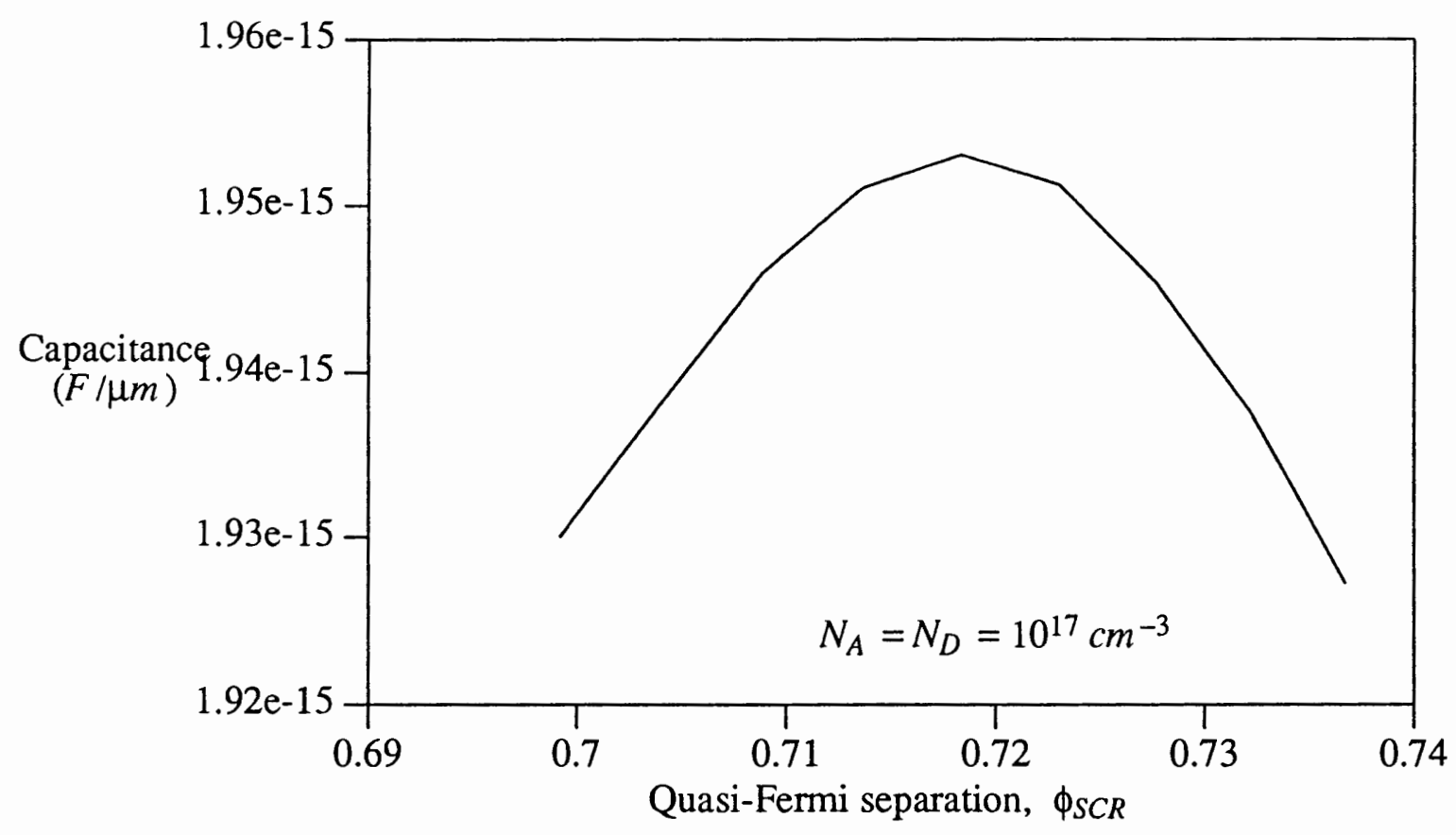

Figure 21.Enlarged peak of Figure 20 to compare the location and value of the peak with that found by Van Den Biesen [15]. 
In this work three types of doping profiles, symmetrical abrupt, asymmetrical abrupt, and linearly graded doping profile will be used and the capacitance will be calculated with respect to the quasi-Fermi level separation. Tekspice is used to generate the the linear graded doping profile for PISCES simulation.

\section{IV.5 PROBLEM IN EXTRACTION OF CAPACITANCE BY PISCES}

As it is erroneous to use the ac small-signal analysis method in PISCES is erroneous to extract the space charge region capacitance at high forward bias, one has to extract $C_{s c r}$ by net charge or net carrier method. For this two methods one has to take some precaution to avoid erroneous result at high forward bias. First of which, the accumulated charge near the contact and second the spill over charge from highly doped side to the low doped side for asymmetrical abrupt junction.

The problem associated with the accumulated charge near the contact is described in detail in section III. To avoid counting this charge into the total charge in the space charge region one has to take the integration limit just outside the SCR. Integration from outside the SCR to the electrical junction of the net charge would yield the correct total charge that contribute to the SCR capacitance. In this work integration limit outside the SCR is considered where the net charge is four orders of magnitude below the peak value of the net charge.

For asymmetrical abrupt doping profile, the net charge method will be erroneous to calculate the total charge in the SCR. Figure 23 shows the net charge distribution for the electron and hole distribution shown in Figure 22. As shown in Figure 23 there is a peak in the n-type side immediately after the metallurgical junction. If the p-type is much more highly doped than the n-type side, holes will start to spill over into the n-type side. 
In the immediate neighborhood of the metallurgical junction of the n-type side, the density of the hole concentration will be greater than the doping concentration $\left(N_{D}\right)$. As PISCES calculates the net charge through Eq. (4.1), the net charge in the neighborhood of the metallurgical junction in the n-type side will be greater than $N_{D}$. For this reason the upper peak value in Figure 23 is higher than $N_{D}$. Although the electrical junction is away from the metallurgical junction, the net charge changes its polarity at the metallurgical junction. To calculate the total space charge one should integrate the net charge from outside the SCR to the electrical junction. As the electrical junction does not coincide with the metallurgical junction for the asymmetrical abrupt junction, integrating the net charge up to the metallurgical junction will be erroneous to calculate the total charge.

For asymmetrical abrupt junction it is not possible to extract the SCR capacitance by net charge method. Instead, net carrier method which deals only with the variation of the net carriers in the SCR is suitable to calculate the SCR capacitance. One needs to integrate the net carriers from outside the SCR to the electrical junction. Derivative of this integrated net carrier yields $C_{s c r}$, as shown in Eq. (4.3). As stated earlier for the problem of the accumulated charge near the contact, the integration limit was set just outside the SCR. For this work, the integration limit was set at a point where the value of the net carrier is about $99.99 \%$ of the equilibrium value. For the asymmetrical abrupt doping profile the electrical junction is function of the applied voltage. At this electrical junction the net carrier density will change its sign.

In Figure 24, the peak of the net carrier concentration shows the position of the electrical junction at $x_{1}$, whereas the metallurgical junction is at $x_{o}$. This electrical junction tends to come closer to the metallurgical junction at higher forward bias, where electrical and hole flow increases considerably. 


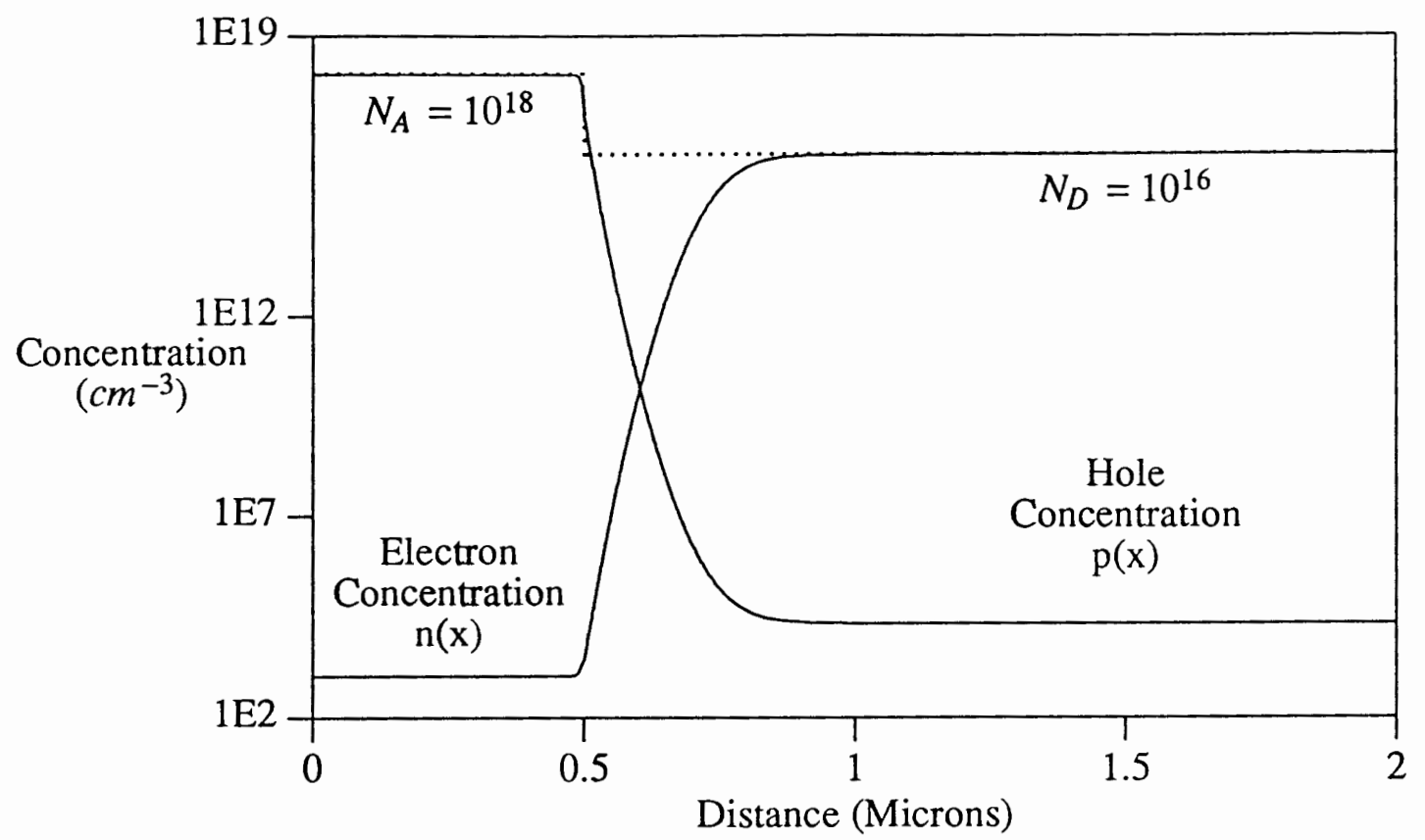

Figure 22.Distribution of $p(x)$ and $n(x)$ for an asymmetrical abrupt junction, $N_{A}=10^{18}$ and $N_{D}=10^{16} \mathrm{~cm}^{-3}$ and a junction depth of 0.5 micron, for $V_{a p p}=0$.

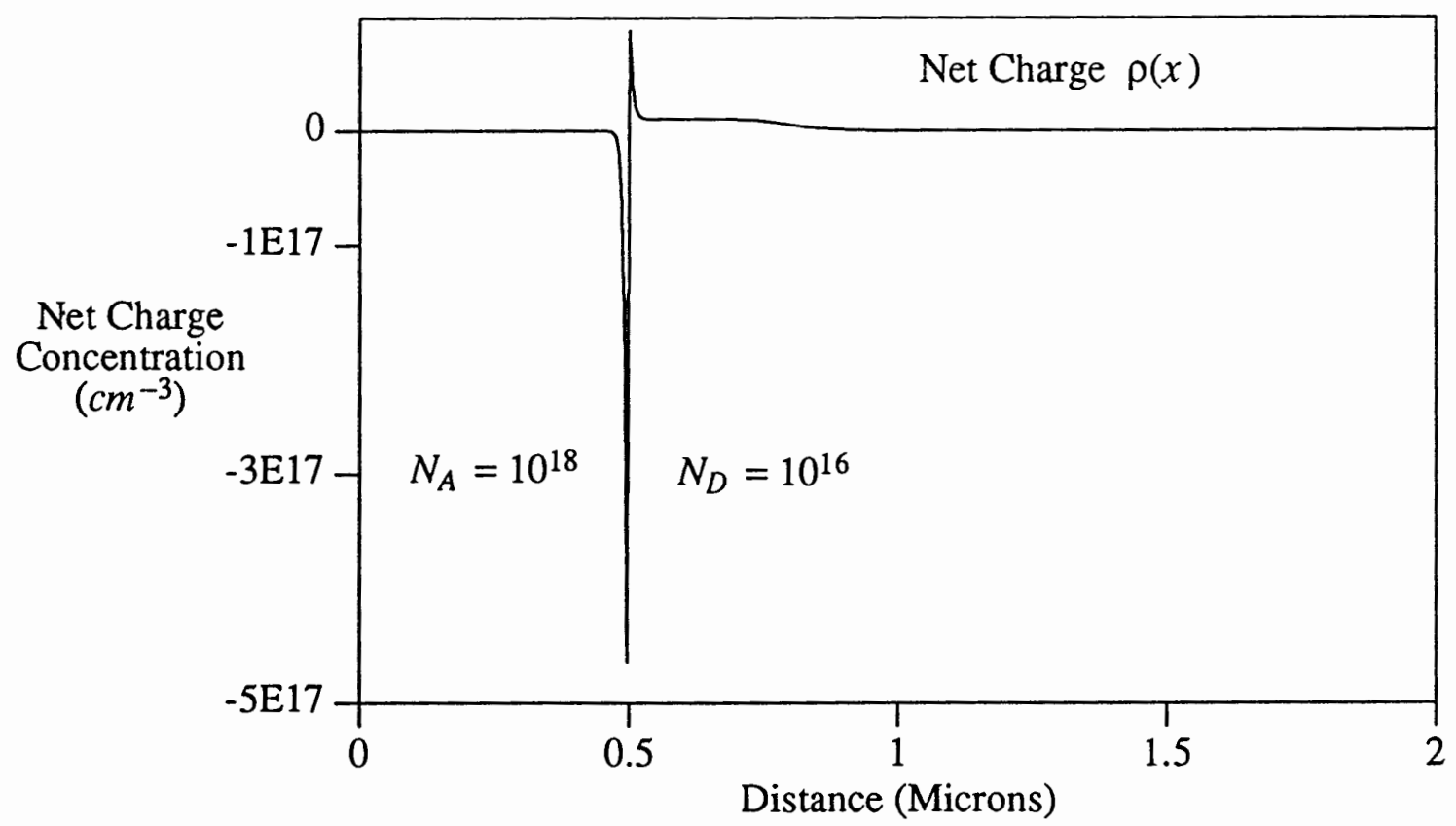

Figure 23.Space charge distribution $\left(\mathrm{cm}^{-3}\right), \quad \rho(x)$, extracted by PISCES for asymmetrical abrupt junction shown in Figure 22, for $V_{\text {app }}=0$. 
For this work the cross over point of the electron and hole concentration (electrical junction) is located for each bias point and the net carrier concentration is integrated from the outside the SCR to the electrical junction.

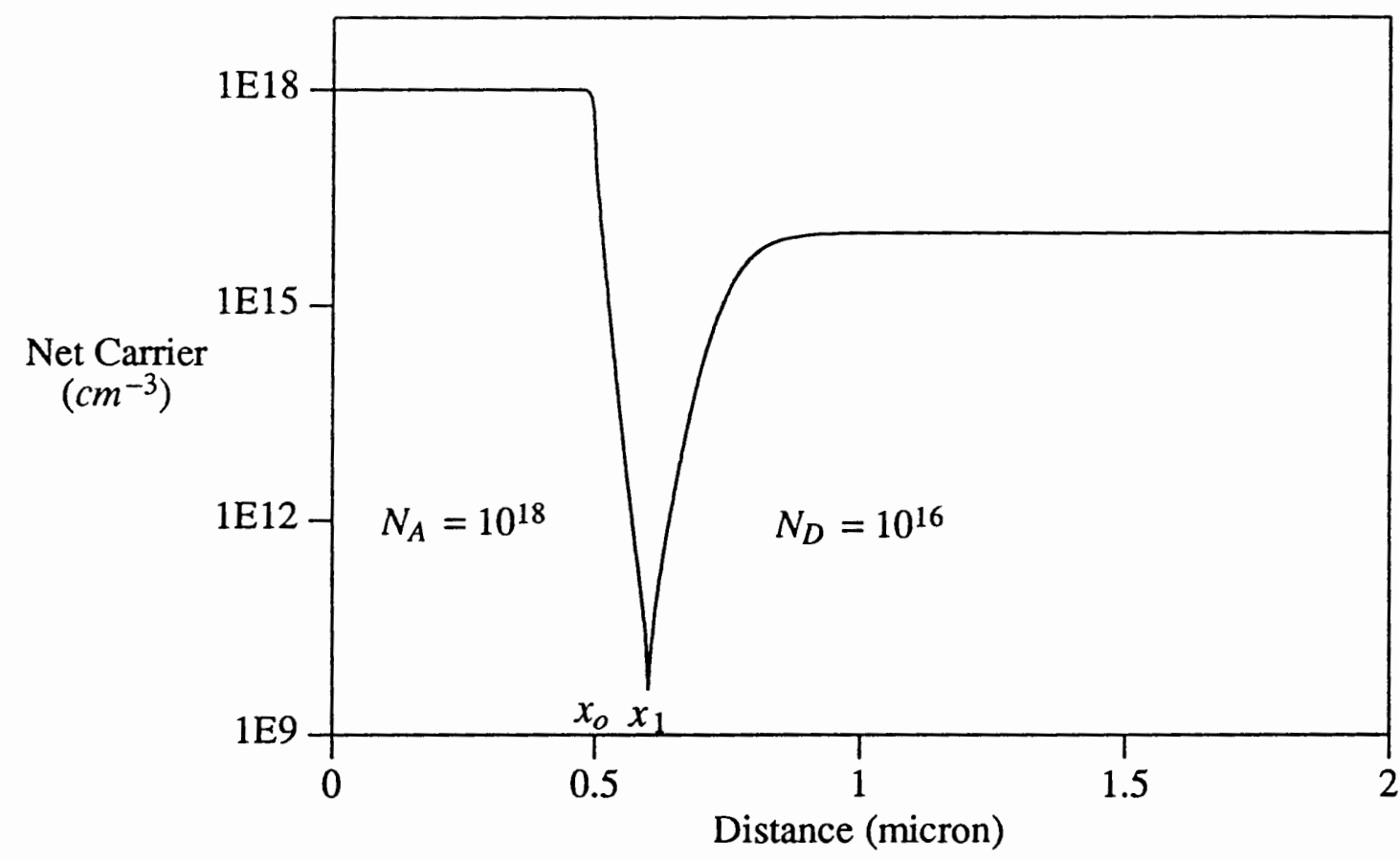

Figure 24.Distribution of net carrier concentration in absolute value, extracted by PISCES for $V_{a p p}=0$, for asymmetrical abrupt junction shown in Figure 22.

The net charge method and the net carrier method both predicts the SCR capacitance accurately as long as the electrical junction coincides with the metallurgical junction, which is true only for symmetrical junction. For asymmetrical junction, the net charge method does not give the accurate capacitance and net carrier method should be used. 


\section{CHAPTER V}

\section{NEW CAPACITANCE MODEL}

\section{V.1 DERIVATION OF A NEW MODEL FOR SCR CAPACITANCE}

According to the depletion approximation the space charge region capacitance becomes infinite when the applied voltage becomes equal to the built-in potential, where as the experiments and computer simulation shows that $C_{s c r}$ has a finite maximum and has a fall off characteristics at higher voltages. Regardless of this discrepancy circuit simulators, such as SPICE, use the depletion approximation model due to its inherent simplicity. A new nonphysical parameter (FC in SPICE) is introduced to avoid infinite capacitance at $V_{a p p}=V_{b i}$. It is important to investigate this discrepancy since the high frequency behavior of a bipolar transistor is very sensitive to the emitter-base space charge region capacitance. According to Tang and Solomon [22], $C_{s c r}$ is an important factor for minimizing the total delay time of the emitter coupled logic gate. Thus a need exists for better characterization of the SCR capacitance for forward bias to meet the requirements of circuit simulation and design.

A new model will be developed in this section and this model will be compared with the results of computer simulations. This model will be verified for three types of doping profiles, symmetrical abrupt, asymmetrical abrupt and linearly graded doping profiles for a wide range of doping concentrations. 
It is evident from Figure 25 that the existing model used in SPICE predicts the SCR capacitance accurately for low forward bias but differs significantly for higher forward bias. Now the task of this work will be to find a suitable model for the SCR capacitance, based on physical justification without introducing any additional input parameters.

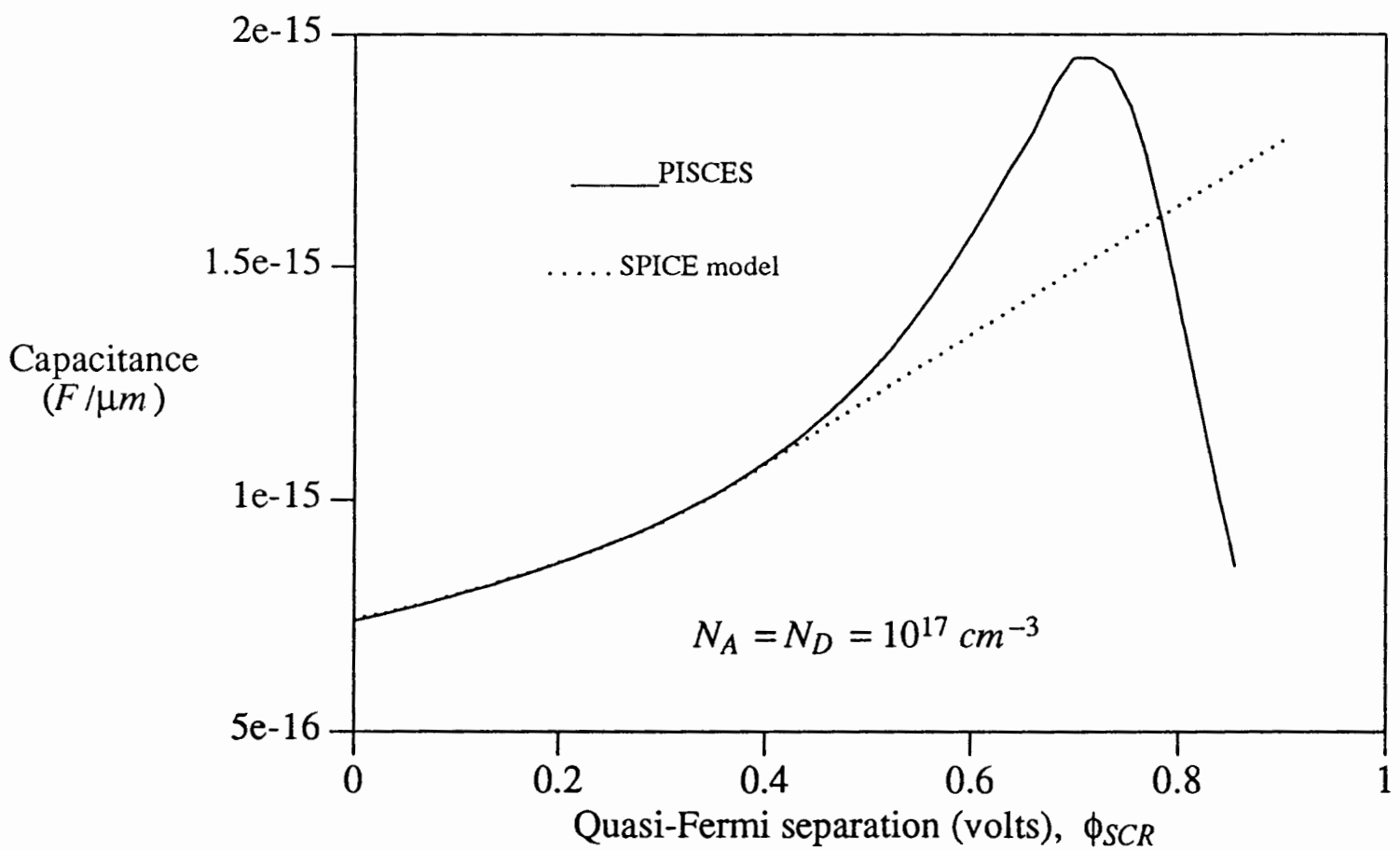

Figure 25.Comparison between the present SPICE model Eq. (1.1) and (1.2) for SCR capacitance and actual capacitance extracted by PISCES.

Eq. (5.1) is used after $2 V_{t}$ correction of $V_{b i}$.

Van Halen [17] introduced a new model for the SCR capacitance Eq. (3.15), which will be repeated here for convenience,

$$
C_{s c r}=\left[\frac{C_{o}}{\left.1-\frac{V_{a p p}}{V_{b i}}+\frac{V_{t}}{2 V_{b i}} \exp \left(\frac{V_{a p p}-V_{b i}}{V_{t}}\right)\right]^{m}}\right.
$$

At reverse and low forward bias Eq. (5.1) reduces to the conventional depletion model Eq. (1.1) and at high forward bias exponential term in the denominator dominates and $C_{s c r}$ decreases exponentially. 
Figure 26 shows that the Van Halen's model has good agreement with the result of PISCES at low forward bias but differs significantly at higher forward bias, specifically the peak value of the capacitance is much higher than the one shown by PISCES. So it is necessary to make changes in Eq. (5.1) so that it matches the peak value of $C_{s c r}$ extracted by PISCES.

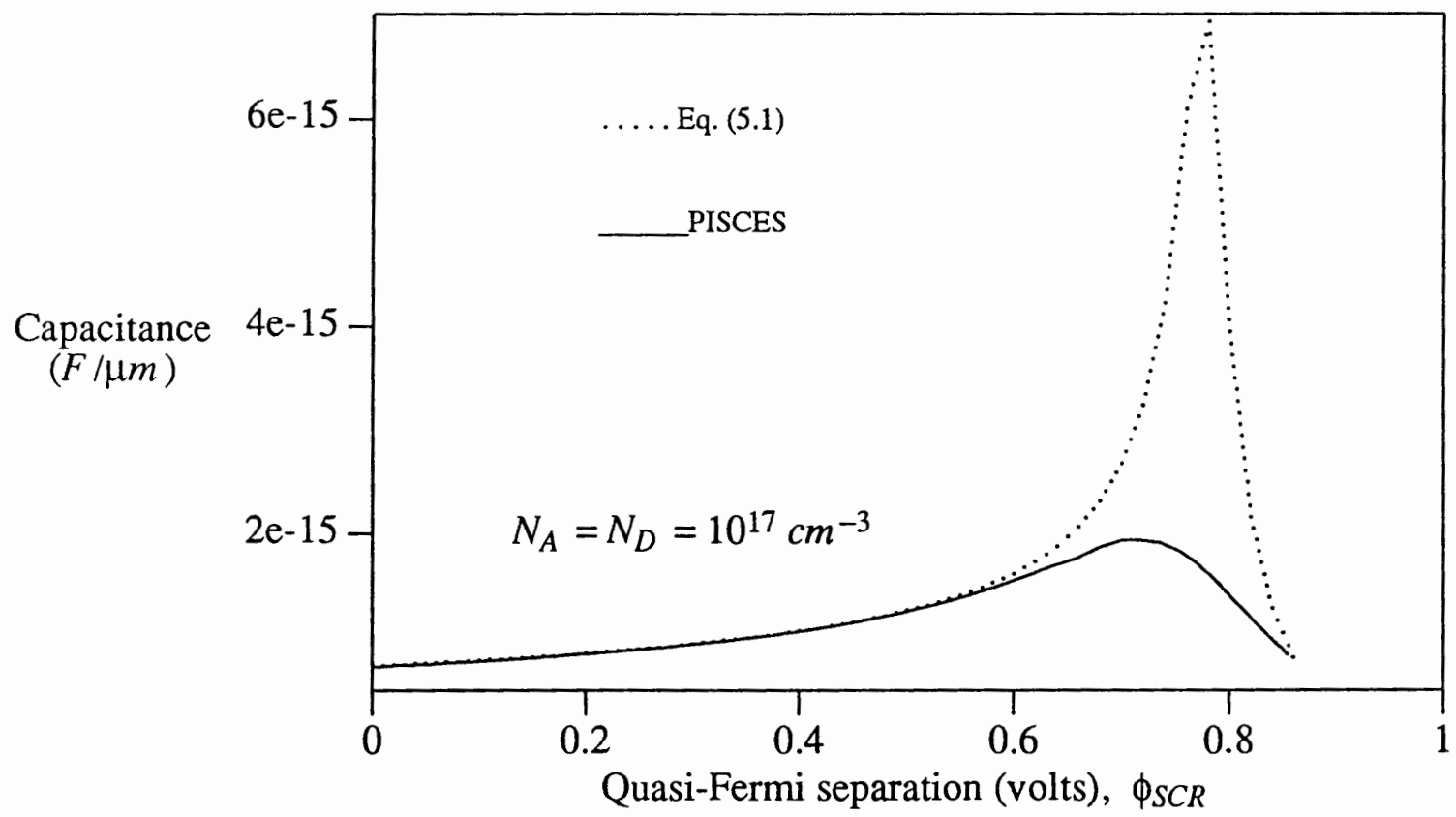

Figure 26.Comparison between the model proposed by Van Halen, Eq. (5.1) and the actual capacitance extracted by PISCES. Eq. (5.1) is used after $2 V_{t}$ correction of $V_{b i}$.

To derive the new model the following things are taken into account; Eq. (5.1) agrees well with the result of PISCES for reverse and low forward bias, it decreases exponentially at high forward bias. Now the value of the peak is dependent on the factor before the exponential term in the denominator of Eq. (5.1). Based on these observations the new model is proposed as

$$
C_{s c r}=\left[\frac{C_{o}}{\left.1-\frac{V_{a p p}}{V_{b i-e f f}}+\frac{(4.7 m+0.7) V_{t}}{V_{b i-e f f}} \exp \left(\frac{V_{a p p}-V_{b i-e f f}}{2 V_{t}}\right)\right]^{m}}\right.
$$


A new term has been introduced in Eq. (5.2) is $V_{b i-e f f}$, which is the effective built-in potential required to calculate the SCR capacitance. Eq. (5.2) reduces to the depletion model Eq. (1.1) for reverse and low forward bias. The pre exponential term in the denominator $\frac{4.7 m+0.7}{2 V_{b i-e f f}}$ has been adjusted so that the peak value of $C_{s c r}$ gives the best fit with the peak value of the PISCES results, for a doping range of $10^{15}$ to $10^{19} \mathrm{~cm}^{-3}$ for symmetrical abrupt and $10^{18}$ to $10^{24} \mathrm{~cm}^{-4}$ for linearly graded junction.

Any practical junction profile must lie within these two extreme cases: linearly graded and abrupt junction. One needs to find the value of $m$ to analyze a particular doping profile. All the three input parameters, $V_{b i-e f f}, C_{o}$ and $m$ can be measured from the experimental data and with these three input parameters one will be able to predict the capacitance behavior at any applied bias.

For symmetrical and asymmetrical abrupt junction $V_{b i-e f f}$ is the reciprocal capacitance squared intercept on the voltage axis and for the linear graded junction $V_{b i-e f f}$ is the reciprocal cubed capacitance intercept on the voltage axis. This $V_{b i-e f f}$ can also be calculated as follows.

For symmetrical abrupt doping profile, according to Chang [6]

$$
V_{b i-e f f}=V_{b i}-2 V_{t}
$$

For asymmetrical abrupt doping profile, according to Kennedy [4]

$$
V_{b i-e f f}=V_{b i}+V_{t}\left(1-\ln \frac{N_{A}}{N_{D}}\right)
$$

For linear graded doping profile, according to Chawla and Gummel [13]

$$
V_{b i-e f f}=V_{g}=\frac{2}{3} V_{t} \ln \frac{a^{2} \varepsilon V_{t}}{8 q n_{i}^{3}}
$$

$C_{o}$ can be calculated from Eq. (3.2) and (3.3) for $V_{a p p}=0$ while replacing $V_{b i}$ 
with $V_{b i-e f f}$.

For symmetrical abrupt junction, according to Chang [6]

$$
C_{o}=\sqrt{\frac{q \varepsilon}{2\left(V_{b i}-2 V_{t}\right)} \cdot \frac{N_{A} N_{D}}{N_{A}+N_{D}}}
$$

For asymmetrical abrupt doping profile, according to Kennedy [4]

$$
C_{o}=\left[\frac{q \varepsilon}{2\left[V_{b i}+V_{l}\left(1-\ln \frac{N_{A}}{N_{D}}\right)\right]} \cdot \frac{N_{A} N_{D}}{N_{A}+N_{D}}\right]^{\frac{1}{2}}
$$

For linear graded junction, according to Chawla and Gummel [13]

$$
C_{o}=\left[\frac{q \varepsilon^{2} a}{8 V_{l} \ln \frac{a^{2} \varepsilon V_{t}}{8 q n_{i}^{3}}}\right]^{\frac{1}{3}}
$$

$m$ is the grading coefficient, 0.5 for abrupt doping profiles and 0.33 for linear graded doping profiles. For this calculation the physical constants are $\varepsilon=1.045 \times 10^{-12} \mathrm{~F} / \mathrm{cm}^{-2}, V_{t}=0.25851$ volts and the effective intrinsic carrier concentration $n_{i e}$ is used,

$$
n_{i e}^{2}=n_{i}^{2} \exp \left(\frac{\Delta E_{g}}{k T}\right)
$$

where $n_{i}=1.4459 \times 10^{10} \mathrm{~cm}^{-3}$ at $T=300^{\circ} \mathrm{K}$, and $\Delta E_{g}$ is the apparent reduction of the bandgap, which is obtained from Slotboom and DeGraaff model [23], as,

where $N$ is the ionized impurity concentration.

$$
\Delta E_{g}=0.009\left[\ln \left(\frac{N}{10^{17}}\right)+\sqrt{\left[\ln \left(-\frac{N}{10^{17}}\right)\right]^{2}+0.5}\right]
$$

Now the new model for $C_{s c r}$ will be compared with the $C_{s c r}$ extracted from PISCES for different doping profiles. To compare this new model for symmetrical abrupt doping profile $V_{b i-e f f}$ and $C_{o}$ will be calculated from Eq. (5.3) and (5.6) respec- 
tively and the value of $m$ will be 0.5 . Figure 27 and Figure 28 shows the comparison of new model with POSCES for symmetrical doping profile of $10^{15}$ and $10^{19} \mathrm{~cm}^{-3}$.

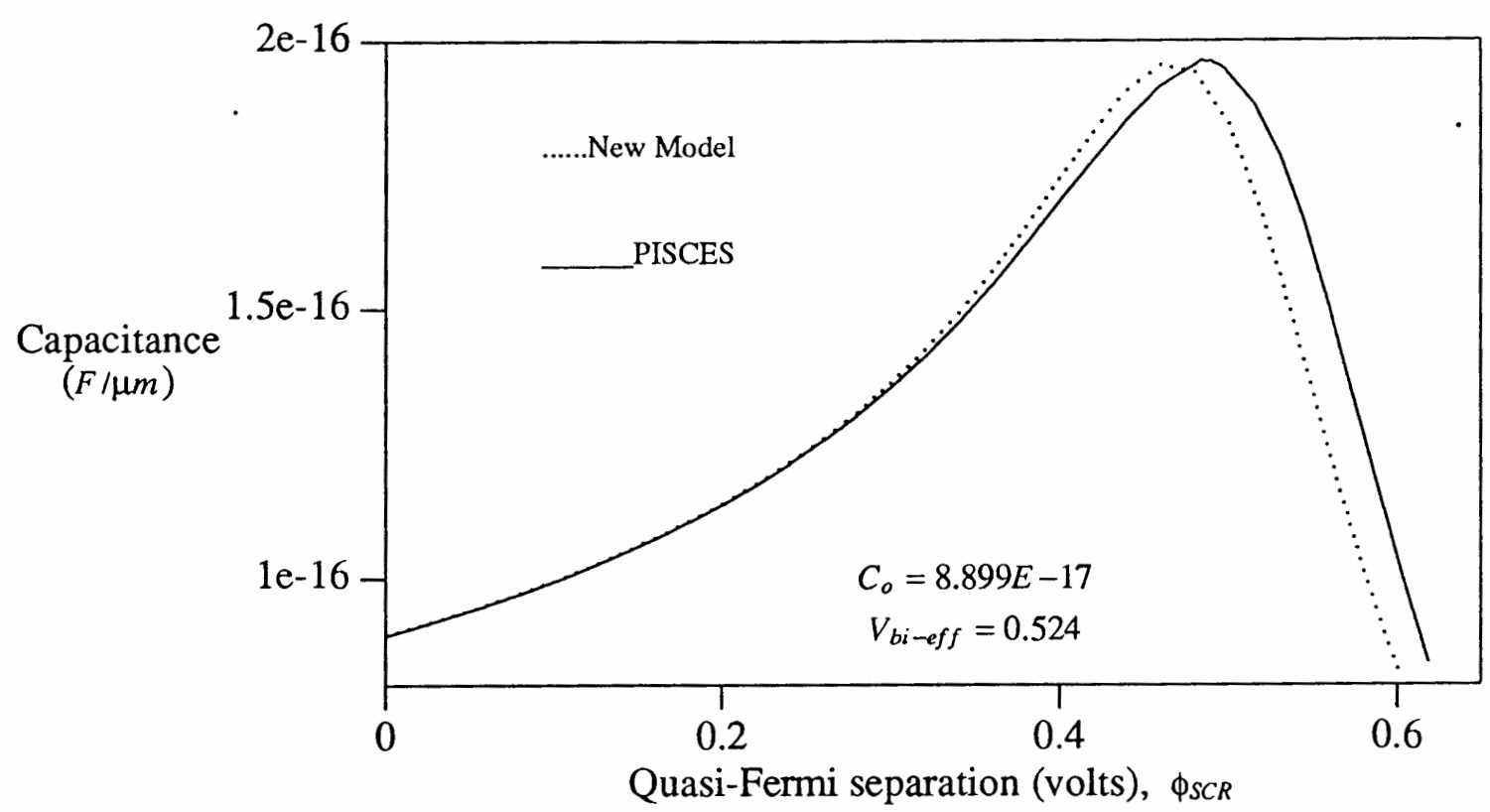

Figure 27.Comparison of the new model Eq. (5.3) with the actual capacitance extracted by PISCES for $N_{A}=N_{D}=10^{15} \mathrm{~cm}^{-3}$.

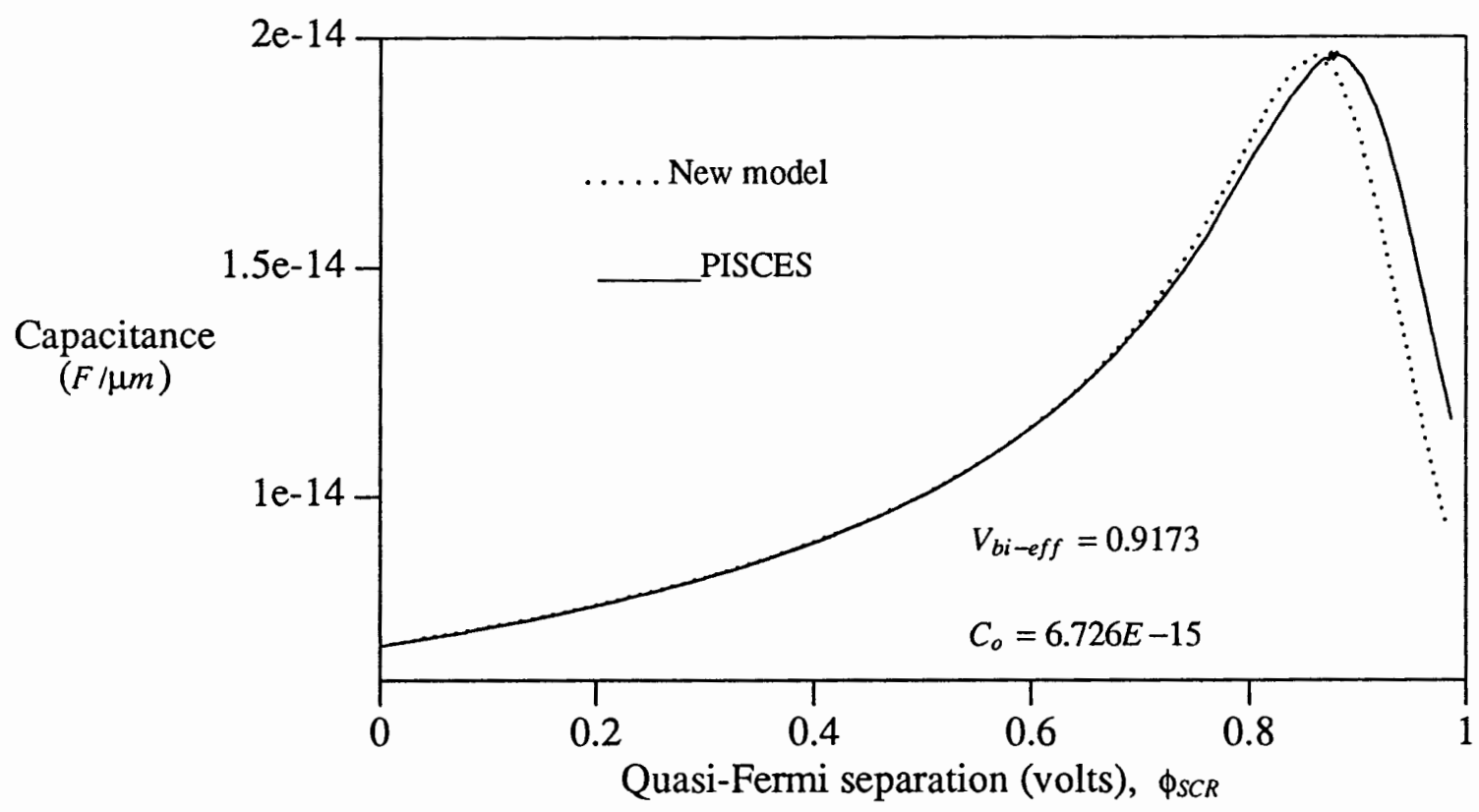

Figure 28.Comparison of the new model Eq. (5.3) with the actual capacitance extracted by PISCES for $N_{A}=N_{D}=10^{19} \mathrm{~cm}^{-3}$. 
Figure 27 and 28 shows an excellent agreement of the new model with the PISCES result at low forward voltage. Capacitance extracted from PISCES follows a inverse squared capacitance versus voltage relationship at low forward voltage which verifies the value of $m=0.5$ for symmetrical abrupt doping profile. At high forward bias the new model has a fall off characteristics with some deviation from the PISCES result.

To compare the new model for linearly graded doping profile, $V_{b i-e f f}$ and $C_{o}$ will be calculated from Eq. (5.4) and (5.7) respectively. Value of $m$ will be 0.333 . Figure 29 and Figure 30 shows the comparison of new model with the capacitance extracted from PISCES for doping gradient of $10^{18}$ and $10^{24} \mathrm{~cm}^{-4}$.

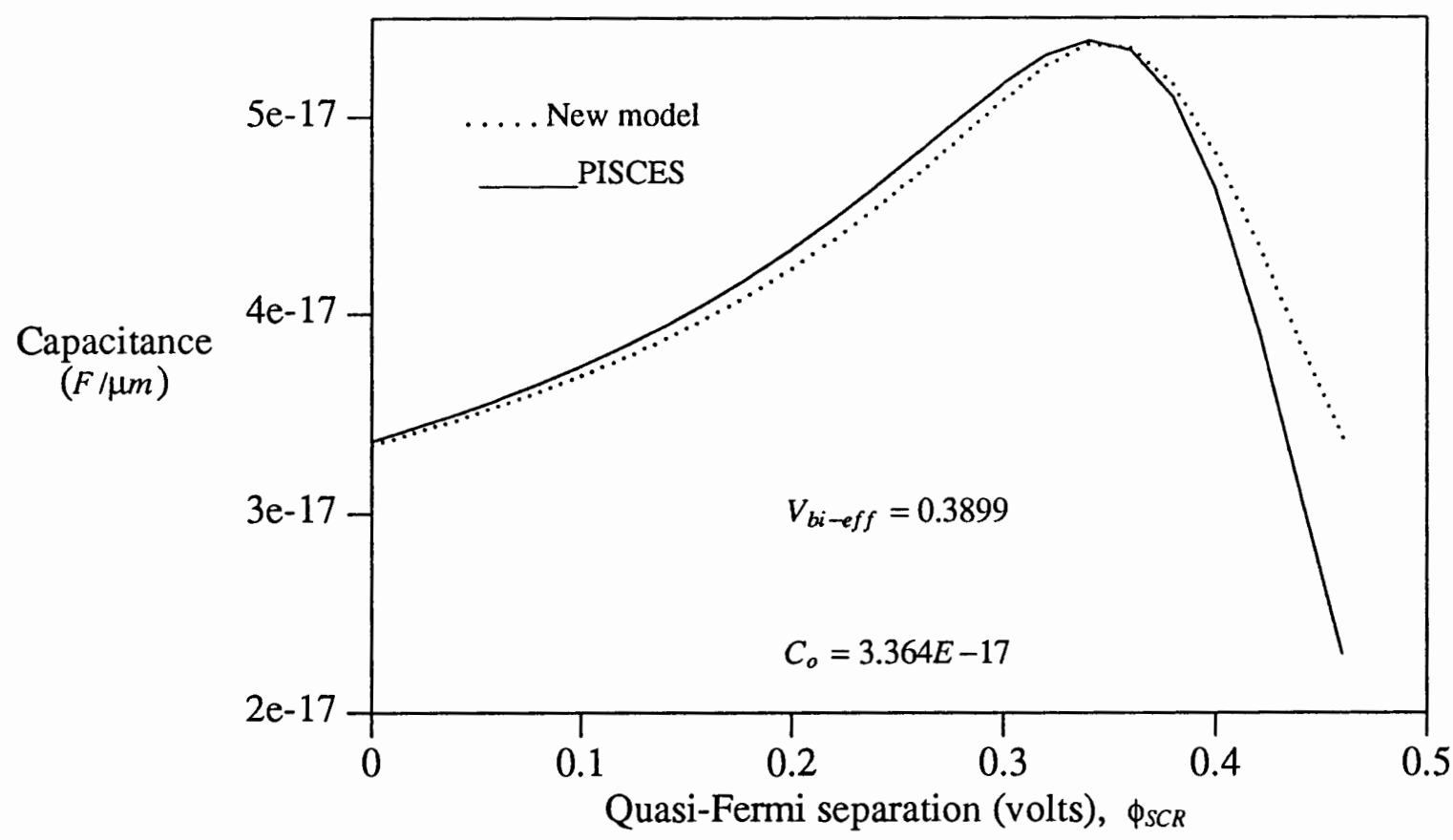

Figure 29.Comparison of the new model Eq. (5.3) with the actual capacitance extracted by PISCES for linear graded profile with $a=10^{18} \mathrm{~cm}^{-4}$. 


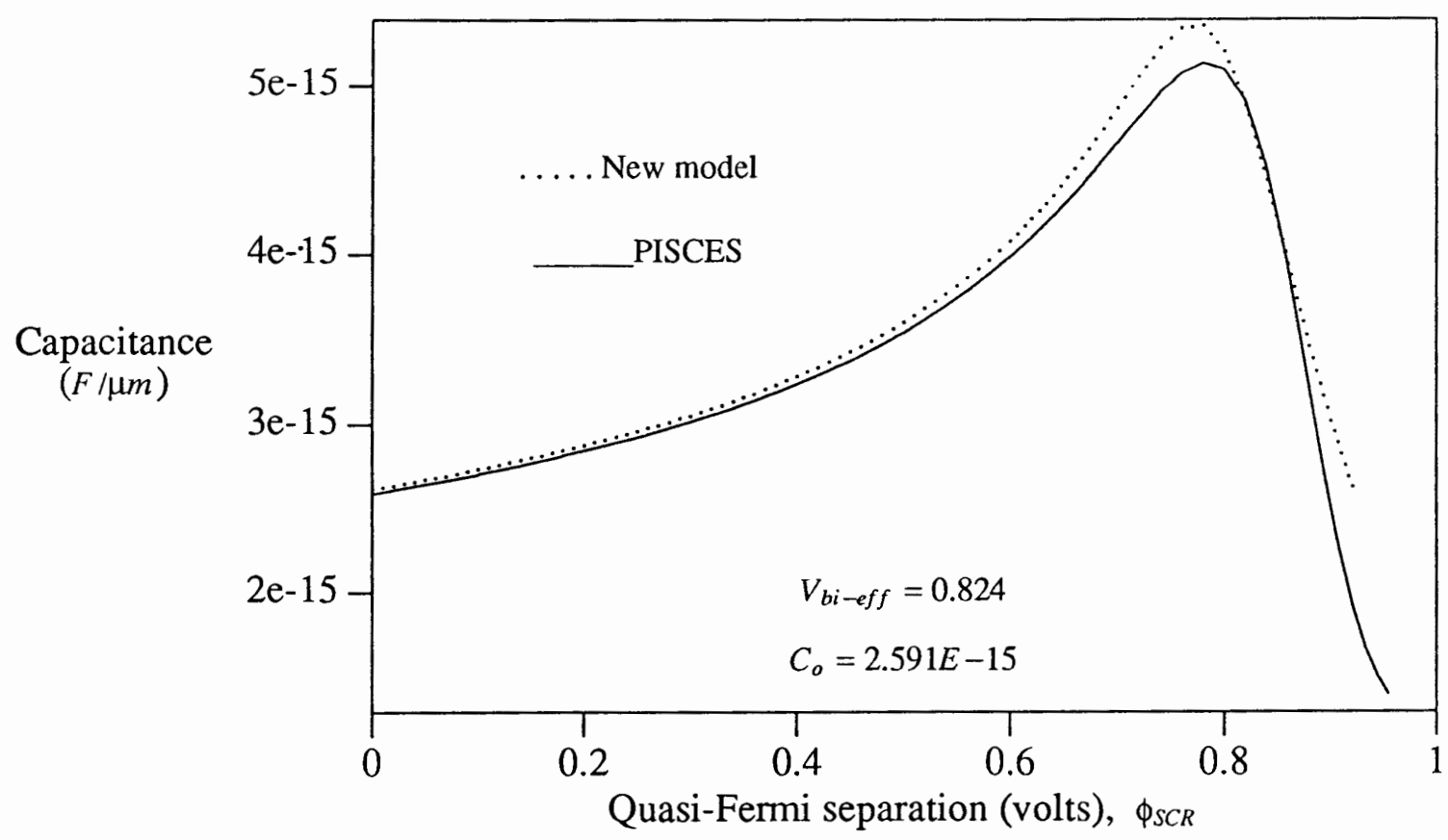

Figure 30.Comparison of the new model Eq. (5.3) with the actual capacitance extracted by PISCES for linear graded profile with $a=10^{24} \mathrm{~cm}^{-4}$.

Figure 29 and 30 shows that the new model has a good agreement at low forward bias with a very little discrepancy. This is due to the assumption of the gradient voltage, which was assumed to be the inverse cubed capacitance versus voltage intercept. In reality this intercept voltage is dependent on the applied voltage as shown by Chawla and Gummel [13]. But Figure 29 and 30 shows that the gradient voltage assumption gives a reasonable fit of the new model to the capacitance extracted by PISCES.

To compare the new model for asymmetrical abrupt doping profile $V_{b i-f f}$ and $C_{o}$ will be calculated from Eq. (5.4) and (5.7) respectively and the value will be 0.5. Figure 31 to Figure 33 shows comparison of new model with PISCES for various asymmetrical abrupt doping profile. 


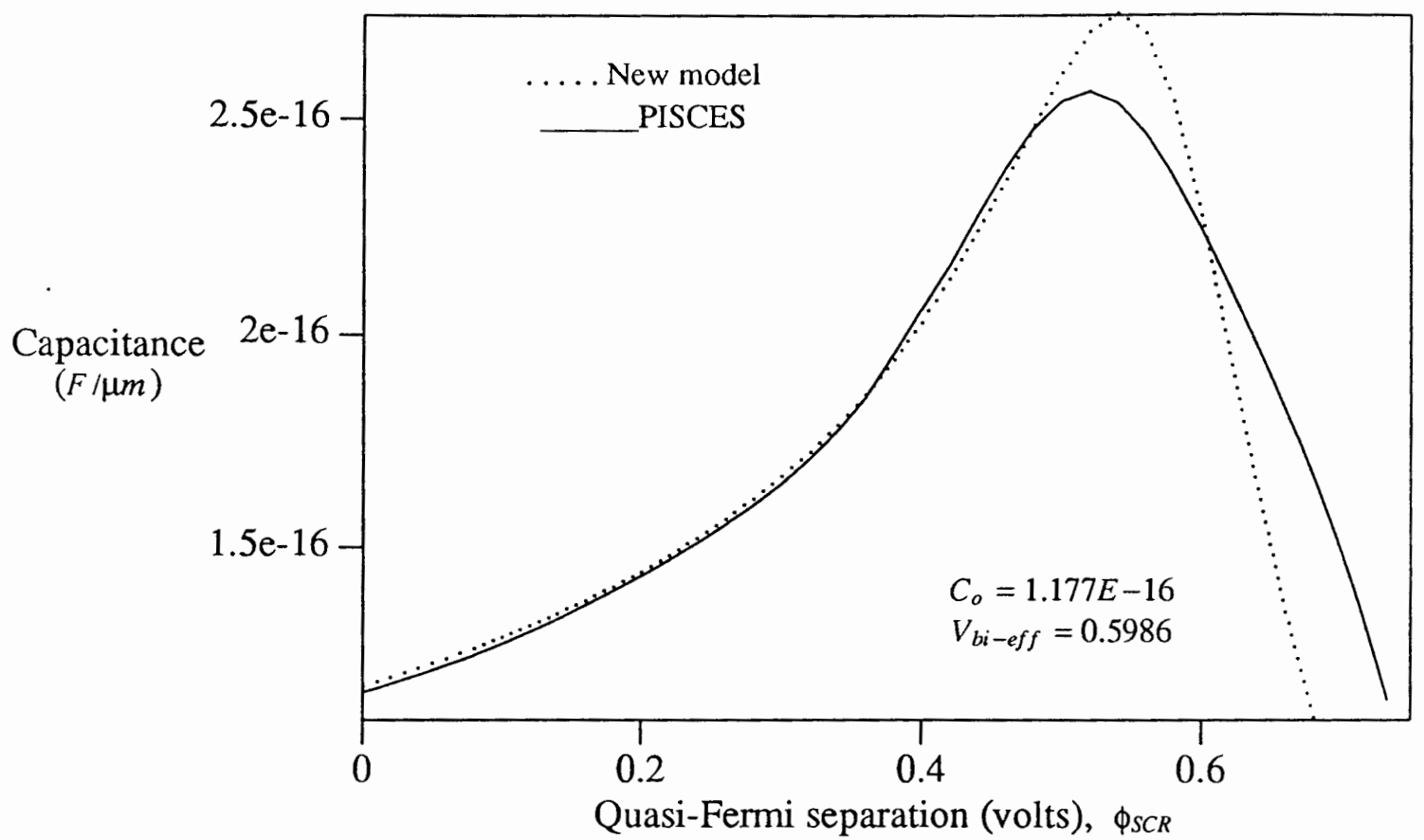

Figure 31.Comparison of the new model Eq. (5.3) with the actual capacitance extracted by PISCES for $N_{A}=10^{17}$ and $N_{D}=10^{15} \mathrm{~cm}^{-3}$.

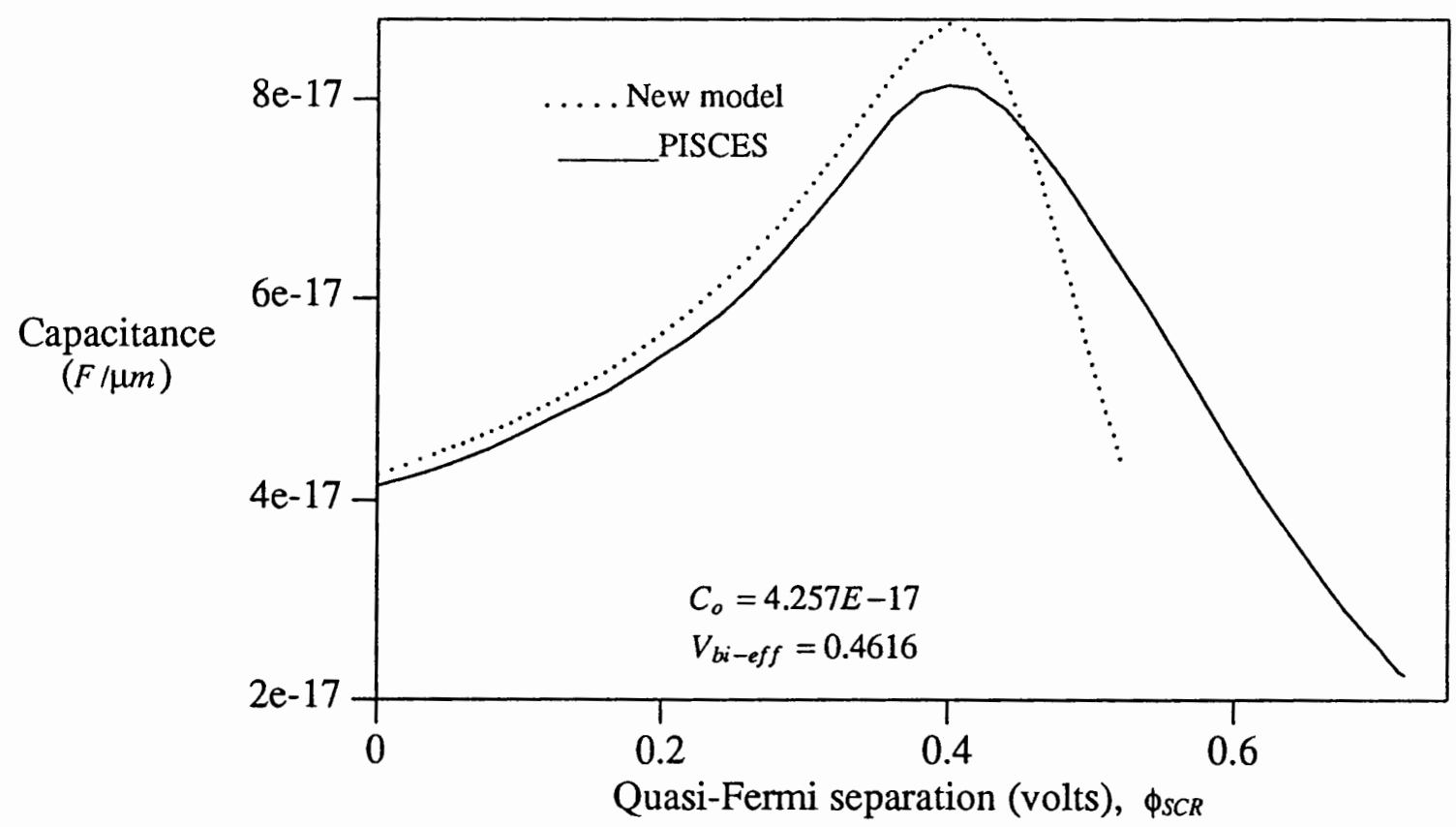

Figure 32. Comparison of the new model Eq. (5.3) with the actual capacitance extracted by PISCES for $N_{A}=10^{18}$ and $N_{D}=10^{14} \mathrm{~cm}^{-3}$. 


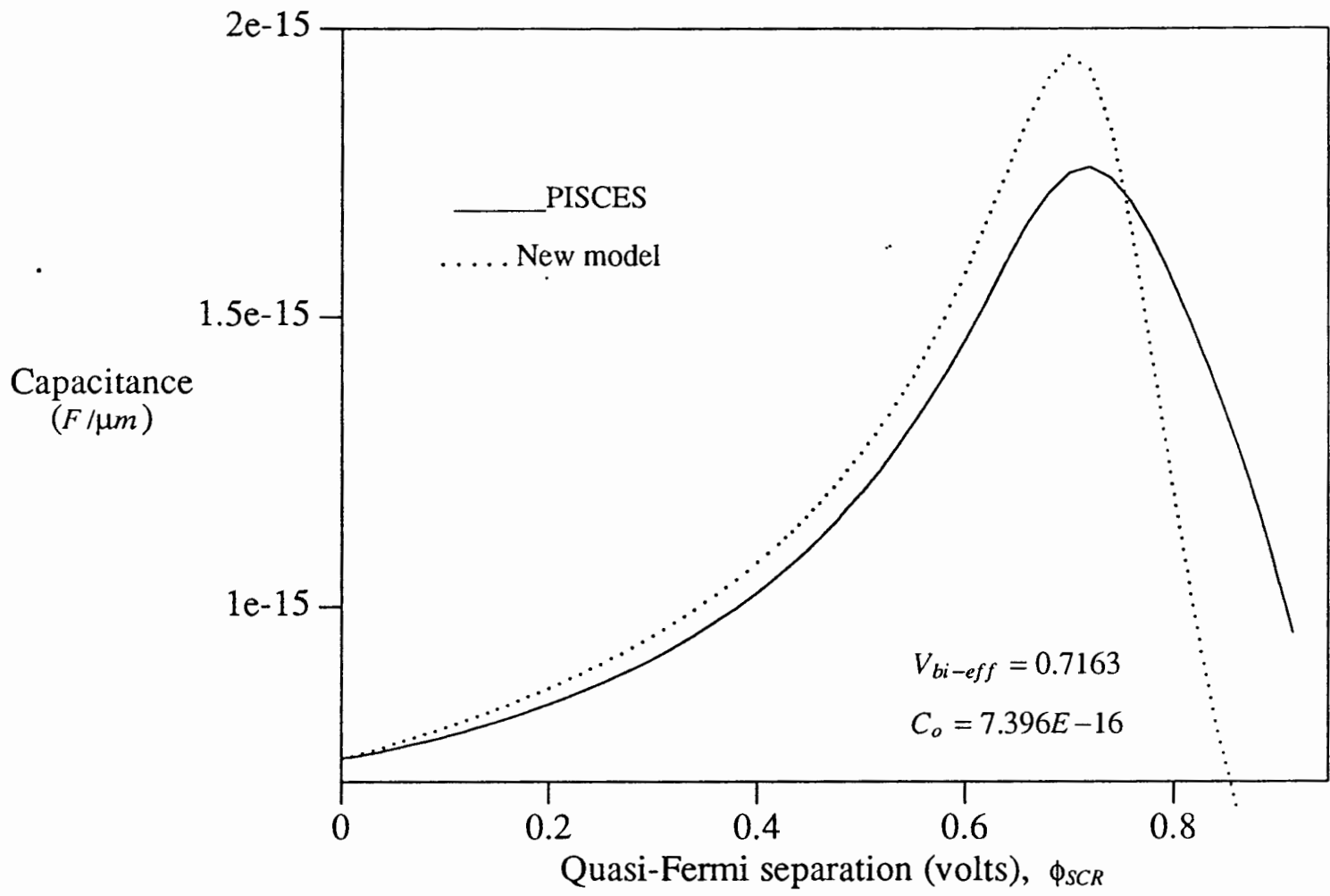

Figure 33. Comparison of the new model Eq. (5.3) with the actual capacitance extracted by PISCES for $N_{A}=10^{19}$ and $N_{D}=5 \times 10^{16} \mathrm{~cm}^{-3}$.

In Figure 31 to Figure 33 the error produced by the new model at low forward bias is due to the calculation of $V_{b i-e f f}$ and $C_{o}$ from $\mathrm{Eq}$ (5.4) and (5.7) respectively. As shown by Kennedy[4] that using Eq. (5.4) to calculate $V_{b i-e f f}$ can produce an error of about $3.5 \%$ at thermal equilibrium. At high forward bias the new model gives a larger error and this error can be minimized by adjusting the value of $m$. As the inverse squared capacitance versus voltage intercept is dependent on the applied voltage, shown by several authors $[7,8]$, the value of $m$ is not exactly equal to 0.5 for asymmetrical abrupt doping profile. Using the correct value of $V_{b i-e f f}$ and $m$ will probably give a better result. 


\section{V.2 COMPARISON OF NEW MODEL WITH PREVIOUS MODEL}

In this section the three most sophisticated models, the Gummel and Poon model, the DeGraaff and Klaassens model and the Van Halen model, that were proposed in the literature will be compared withe the new model on the basis of PISCES results.

For Gummel and Poon (GP) model Eq. (3.17), DeGraaff and Klaassen (GK) model derivative of Eq. (3.18) and for Van Halen (VH) model Eq. (3.15) are considered. For the purpose of testing these models $C_{o}, V_{b i}$ and $m$ are given their theoretical values. Where $C_{o}=C_{s c r}\left(V_{a p p}=0\right), V_{b i}=V_{b i-e f f}$ and $m$ will be 0.5 for abrupt and 0.33 for linear graded doping profile.

Figure 34 shows the comparison between GP, GK and the new model with the result of PISCES for symmetrical abrupt doping profile. GK model shows a good agreement with PISCES. In Figure 35 GP and GK model is compared with the new model and Figure 36 shows a comparison of VH model with the PISCES result for linear graded doping profile with $a=10^{22} \mathrm{~cm}^{-4}$. GK model shows a better agreement than the GP model. Although the $\mathrm{VH}$ model predicts a higher peak value of $C_{s c r}$, it uses only three input parameters. For both linear graded and abrupt junction GK model shows very good agreement with PISCES result as well as with the new model, with only difference that GK model requires four input parameter compared to three in the new model. Peak of the Figure 34 is enlarged in Figure 37 to compare the behavior of the models near the peak value of $C_{s c r}$. 


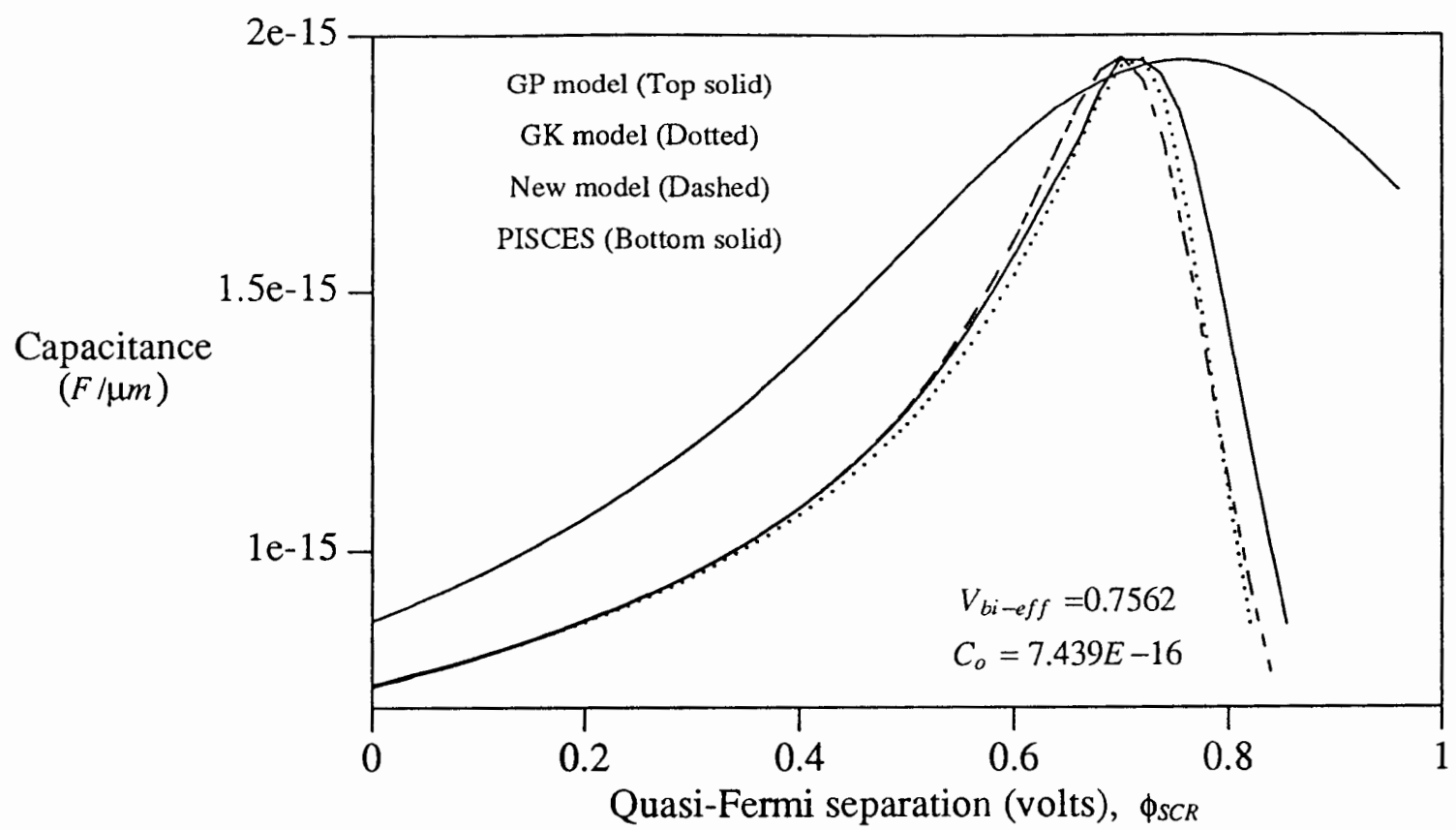

(a)

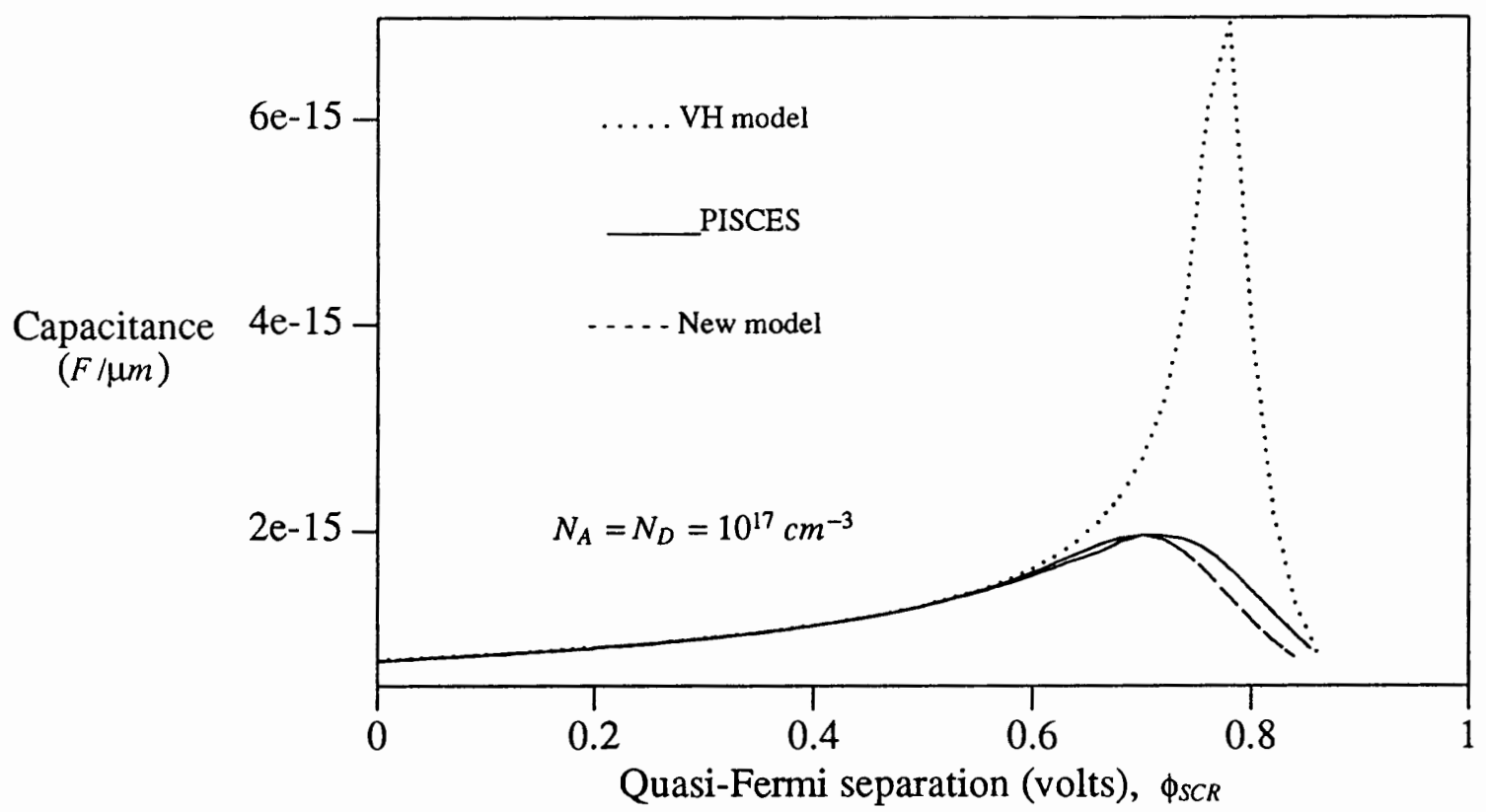

(b)

Figure 34.Comparison of the new model with VH, GP and GK model. For GP model $\mathrm{b}=0.3387$, and GK model $\mathrm{K}=0.00859$ is used for $N_{A}=N_{D}=10^{17} \mathrm{~cm}^{-3}$ on the basis of PISCES result. 


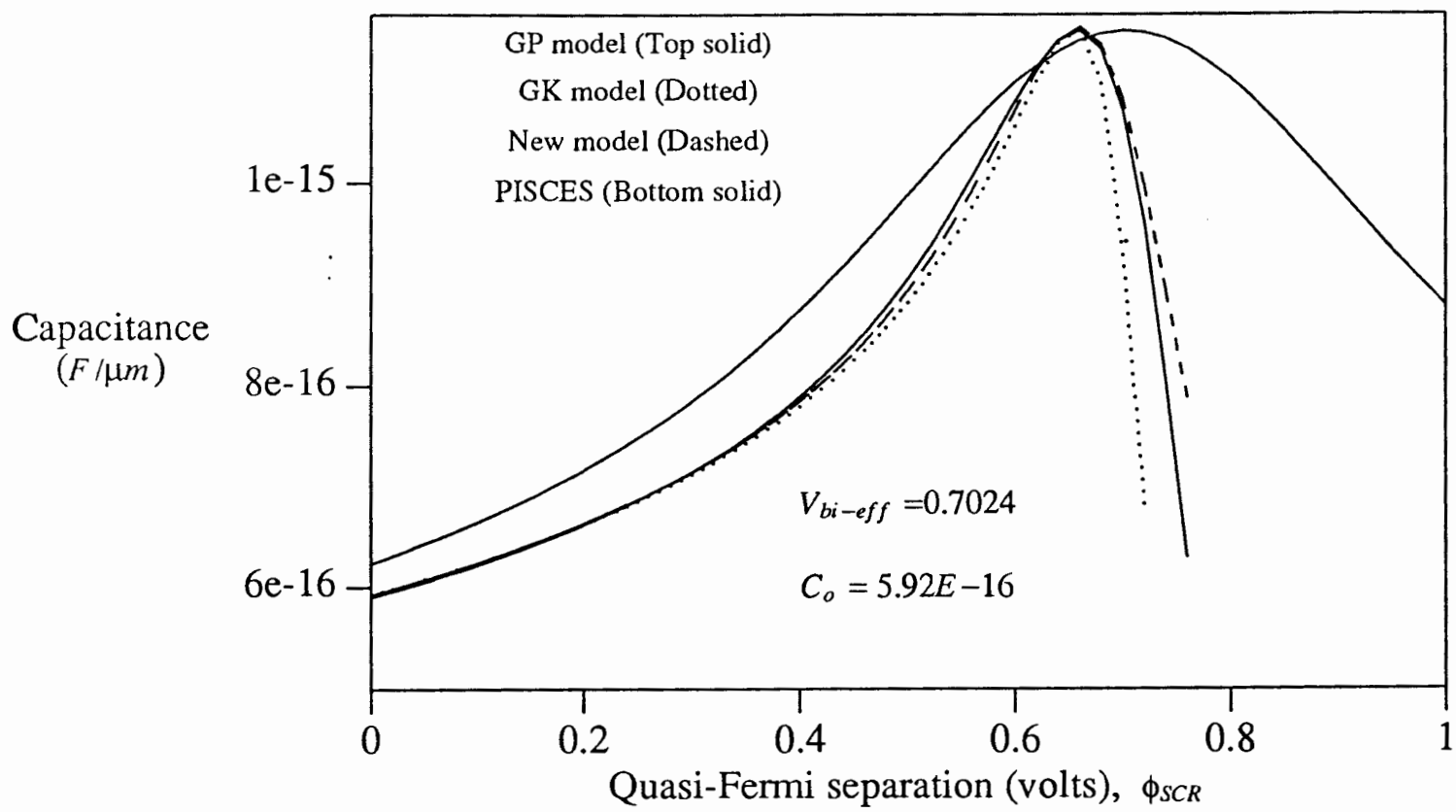

Figure 35.Comparison of the GP and GK model and the new model with PISCES result for linear graded profile with $a=10^{22} \mathrm{~cm}^{-4}$. For GP model $\mathrm{b}=0.207$ and for $\mathrm{GK}$ model $\mathrm{K}=0.0046$ is used.

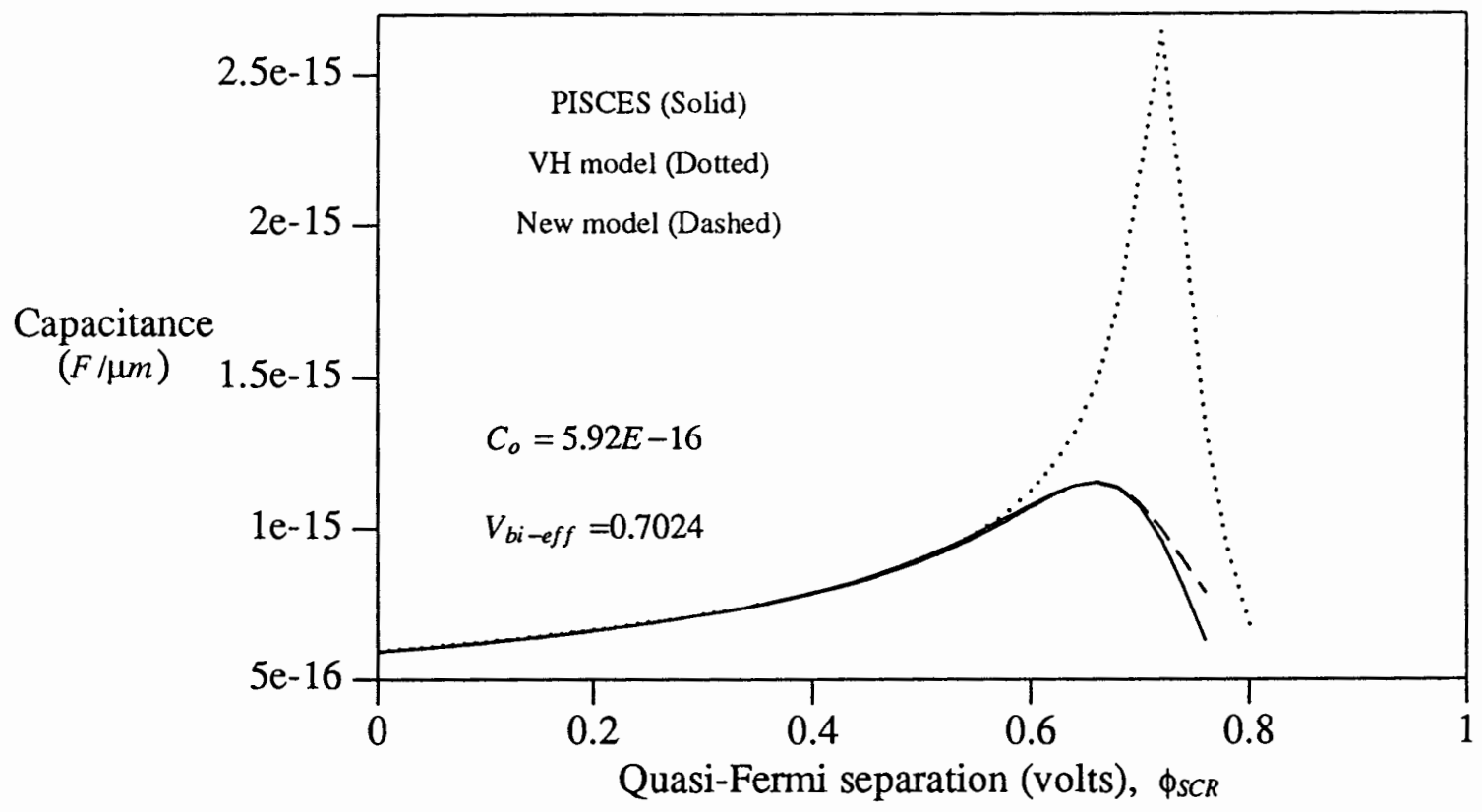

Figure 36.Comparison of the VH model and the new model with PISCES result for linear graded profile with $a=10^{22} \mathrm{~cm}^{-4}$. 


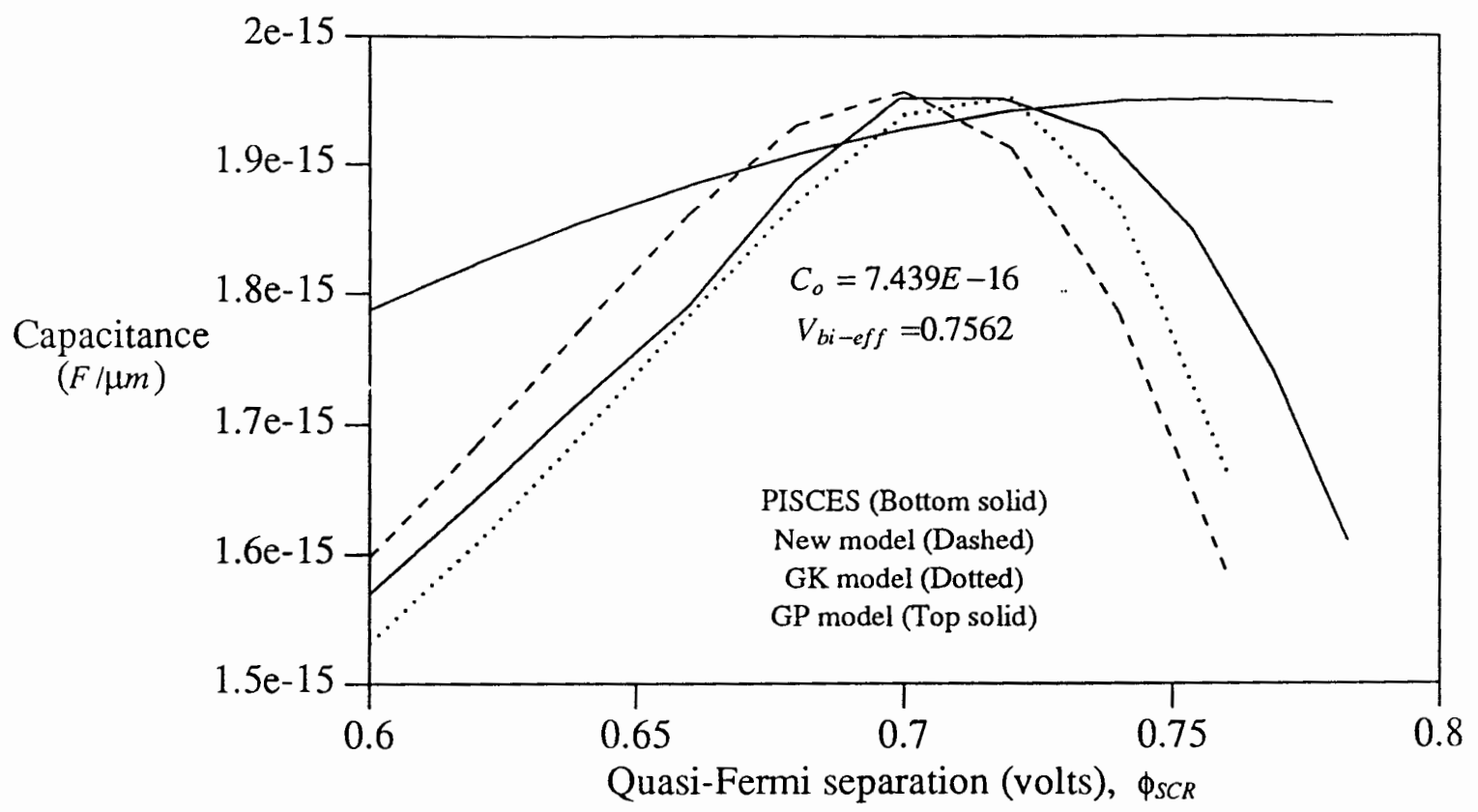

Figure 37.Enlarged peak of Figure 34. Comparison of the new model with GP $(\mathrm{b}=0.3387)$ and GK $(\mathrm{K}=0.00859)$ model for $N_{A}=N_{D}=10^{17} \mathrm{~cm}^{-3}$ on the basis of PISCES result. 


\section{CHAPTER VI}

\section{CONCLUSION}

A new model for SCR capacitance has been derived which is free of singularity and applicable for any applied bias. This new model is suitable for circuit simulators and takes only three input parameters, $C_{o}, V_{b i-e f f}$ and $m$.

For the symmetrical abrupt doping profile, the inverse squared capacitance intercept on voltage axis does not depend on the applied voltage. So, Eq. (5.3) for the calculation of $V_{b i-e f f}$ and Eq. (5.6) for calculation of $C_{o}$ gives fairly accurate result when one compares this result with that obtained from PISCES. Figure 27 and 28 shows that Eq. (5.2) has excellent agreement with the result of PISCES at lower forward bias. At high forward bias where $C_{s c r}$ approaches its peak value, the new model shows a little deviation from the PISCES result. This difference was found to be within $4 \%$ when $C_{s c r}$ is around its peak value for a doping profile of $10^{15}$ to $10^{19} \mathrm{~cm}^{-3}$. After the peak the difference is significant, but the diffusion capacitance will dominate in this region.

For the asymmetrical abrupt junction, the inverse squared capacitance intercept on voltage axis is dependent on the applied voltage as shown by several authors $[7,8]$. Kennedy's [4] model, Eq. (3.9) gives a maximum of 3.5\% error at thermal equilibrium. Hence calculation of $V_{b i-e f f}$ from Eq. (5.4) and $C_{o}$ from Eq. (5.7) is not expected to be as accurate as for the symmetrical abrupt junction. Eq. (5.4) and (5.7) are used in this capacitance calculation as $V_{b i-e f f}$ and $C_{o}$ can be calculated from the device parameters. Comparing Eq. (5.2) with the PISCES result for asymmetrical abrupt junction, the error was found to be within $3 \%$ for low forward bias and at high forward bias where 
$C_{s c r}$ approaches its peak, the error was found to be within $10 \%$.

For the linearly graded doping profile, the inverse cubed capacitance intercept on the voltage axis is also a function of the applied voltage as shown by Chawla and Gummel [13], but they showed that the gradient voltage Eq. (5.5) is a good approximation of the intercept voltage. The difference between the intercept voltage obtained from PISCES and the gradient voltage was found to be less than the thermal voltage. Figure 29 and 30 shows that Eq. (5.2) gives a small error at moderate forward bias, this is because of the assumption of the gradient voltage. At high forward bias, where the peak of the $C_{s c r}$ occurs, the error was less than $5 \%$ for a doping gradient of $10^{18}$ to $10^{24} \mathrm{~cm}^{-4}$.

This new model gives a very good estimate of the SCR capacitance up to the peak value of the $C_{s c r}$. At higher forward bias where $C_{s c r}$ decreases exponentially, the error associated with Eq. (5.2) is large but the diffusion capacitance is orders of magnitude higher than the $C_{s c r}$. 


\section{REFERENCES}

[1] K. M. Van Vliet, "High injection theories of the p-n junction in the charge neutrality approximation." Solid State Electron, vol. 9, pp. 185, 1966.

[2] W. Shockley, "Theory of $\mathrm{p} / \mathrm{n}$ junction semiconductors and $\mathrm{p} / \mathrm{n}$ junction transistors." Bell Syst. Tech. J., vol. 28, pp. 435-489, July 1949.

[3] S. P. Morgan and F. M. Smits, "Potential distribution and capacitance of graded $\mathrm{p} / \mathrm{n}$ junctions," Bell Syst. Tech. J., vol. 39, pp. 1573-1602, Nov. 1960.

[4] D. P. Kennedy, "A mathematical study of space charge capacitance for an abrupt pn semiconductor junction," Solid State Electron, vol. 20, pp. 311-319, 1977. Figure 3 of page 314 is reproduced with written permission from Pergamon Press Lto. Headington Hall, Oxford, 0X3, 0BW, UK.

[5] D. R. Muss, "Capacitance measurement on alloyed Indium-Germanium junction diodes." J. App. Phy., vol. 26, No. 12, pp. 1514-1517, Dec. 1955.

[6] Y. F. Chang, "Capacitance of p-n junctions: Space charge capacitance," J. App. Phy., vol. 37, No. 6, pp. 2337-2342, May 1966.

[7] H. K. Gummel and D. L. Scharfetter, "Depletion layer capacitance of p-n junctions," J. App. Phy., vol. 38, No. 5, pp. 2148-2153, Apr. 1967.

[8] W. Nuyts and R. Van Overstraeten, "Analytical expression for electric field and capacitances of symmetrical and asymmetrical abrupt $\mathrm{p}-\mathrm{n}$ junctions," J. App. Phy., vol. 42, No. 12, pp. 5109-5116, Nov. 1971.

[9] Hans P. Kleinknecht, "Space charge capacitance of asymmetric abrupt p-n junction." J. App. Phy., vol. 38, pp. 3034-3035, 1967.

[10] C. T. Sah, "Effects of electrons and holes on the transition layer characteristics of linear graded p/n junctions," Proc. IRE, vol. 49, pp. 603-618, 1961.

[11] R. M. Sirsi and A. R. Boothroyd, "Characterization of space charge properties of a linearly graded $\mathrm{p}-\mathrm{n}$ junction by approximate 'regional' analysis method." IEEE Trans. Electron Devices, vol. 23, No. 3, pp. 348-353, March 1976.

[12] Juin J. Liou and Fredrik A. Lindholm, "Forward voltage capacitance and thickness of p-n junction space charge regions." IEEE Trans. Electron Devices, vol. ED-34, No. 7, pp. 1571-1579, July 1987. 
[13] B. R. Chawla and H. K. Gummel, "Transition region capacitance of diffused p-n junctions." IEEE Trans. Electron Devices, vol. ED-18, No. 3, pp. 178-195, March 1971.

[14] Fredrik A. Lindholm, "Simple phenomenological modeling of transition region capacitance of forward biased $\mathrm{p}-\mathrm{n}$ junction diodes and transistor diodes." J. App. Phy., vol. 53, No. 11, pp. 7606-7608, Nov. 1982.

[15] J. J. Van Den Biesen, "p/n junction capacitance part I: the depletion capacitance." Philips J. Res., vol. 40, pp. 88-102, 1985.

[16] J. J. Van Den Biesen, "p/n junction capacitance part II: the neutral capacitance." Philips J. Res., vol. 40, pp. 103-113, 1985.

[17] Paul Van Halen, "A new semiconductor diode space charge layer capacitance model." IEEE BCTM, 1988, Paper 8.2, pp. 168-171

[18] Toshio Misawa, "A note on the extended theory of the junction transistor." J. Phys. Soc. Japan, Vol. 10, No. 7, pp. 728-739, July, 1956.

[19] J. J. H. Van Den Biesen, "Modeling the inductive behavior of short base p-n junction diodes at high forward bias, Solid State Electronics, vol. 33, No. 11, pp. $1471-1476,1990$.

[20] H. K. Gummel and H. C. Poon, "Modeling of emitter capacitance." Proc. IEEE, vol. 57, pp. 2181-2182, 1969.

[21] H. C. de Graaff and F. M. Klaassen, "Compact transistor modeling for circuit design." Springer-Verlag Wien, New York, 1990.

[22] D. D. Tang and P. M. Solomon, "Bipolar transistor design for optimized powerdelay logic circuits," IEEE J. Solid-State Circuits, vol. SC-14, pp. 679-684, Aug. 1979.

[23] J. W. Slotboom and H. C. de Graaff, "Measurement of bandgap narrowing in Si bipolar transistors," Solid State Electronics, vol. 19, pp. 857-862, 1976. 